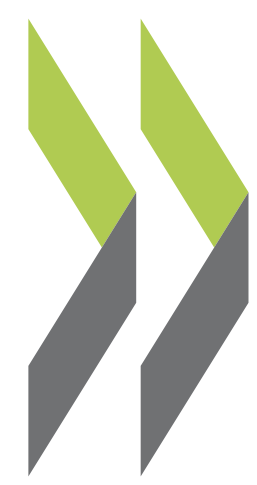

OECD Local Economic and Employment Development (LEED) Papers 2020/10

Un'indagine sulle politiche per il telelavoro: Governare lo sviluppo occupazionale OECD ed economico locale ai tempi del lavoro a distanza 
Serie OCSE sullo Sviluppo Occupazionale ed Economico Locale (LEED)

\title{
Un'indagine sulle politiche per il telelavoro
}

\section{Governare lo sviluppo occupazionale ed economico locale ai tempi del lavoro a distanza}

\begin{abstract}
Questo documento esplora e classifica alcune delle politiche più comuni tra i responsabili politici nazionali, regionali e locali durante o prima della pandemia di COVID-19 per rendere praticabile, promuovere e sfruttare al meglio il telelavoro. Vengono inoltre analizzate alcune misure miranti ad attrarre e trattenere in determinati territori i lavoratori e gli imprenditori che operano a distanza. L'attuale crisi ha comportato, tra gli altri aspetti, una sperimentazione di massa del telelavoro, senza precedenti per dimensioni e portata. La transizione verso un modello di telelavoro diffuso su vasta scala e su base permanente avrebbe profonde implicazioni per la geografia del lavoro a livello locale. Le PMI potrebbero essere meno attrezzate delle grandi imprese per affrontare tale cambiamento. Le politiche pubbliche possono assumere un ruolo cruciale nel trasformare il telelavoro in un'opportunità per tutti, e ridurre il rischio di un potenziale ampliamento delle disparità preesistenti tra persone, territori e aziende.
\end{abstract}

Codici JEL: J28, J62, J68, J88, R11

Parole chiave: telelavoro, occupazione locale, PMI, geografia del lavoro, futuro del lavoro

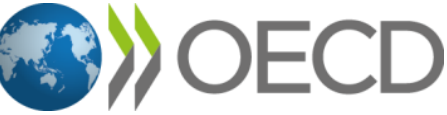




\section{L'OCSE}

L'OCSE è un'organizzazione intergovernativa multidisciplinare cui aderiscono 37 Paesi membri e che coinvolge nel suo lavoro un numero crescente di Paesi non membri da tutte le regioni del mondo. La missione principale dell'Organizzazione è oggi quella di aiutare i governi a cooperare per promuovere un'economia globale più forte, più pulita e più equa. Attraverso la sua rete di 250 comitati e gruppi di lavoro specializzati, I'OCSE fornisce un ambiente in cui i governi confrontano le pratiche di policy, cercano risposte a problemi comuni, identificano le buone pratiche e coordinano le politiche nazionali e internazionali. Per ulteriori informazioni: www.oecd.org.

\section{Serie OCSE sullo Sviluppo Occupazionale ed Economico Locale (LEED)}

La serie di documenti di lavoro del Programma per lo Sviluppo Occupazionale ed Economico Locale dell'OCSE (LEED) presenta idee innovative ed esempi pratici su come promuovere lo sviluppo locale e la creazione di posti di lavoro. L'ampia gamma di tematiche affrontate include l'occupazione e le competenze, l'imprenditorialità, l'economia sociale e l'innovazione sociale, la cultura e il capacity building a livello locale. La serie mette in particolare evidenza le politiche a sostegno di territori e gruppi sociali svantaggiati, come le persone poco qualificate, i disoccupati, i migranti, i giovani e gli anziani.

II presente documento è pubblicato sotto la responsabilità del Segretario Generale dell'OCSE. Le opinioni espresse e le argomentazioni utilizzate non riflettono necessariamente le opinioni ufficiali dei Paesi membri dell'OCSE.

Questo documento è stato autorizzato alla pubblicazione da Lamia Kamal-Chaoui, Direttrice del Centro OCSE per l'Imprenditorialità, le PMI, le Regioni e le Città.

Il presente documento, così come i dati statistici e la mappa in esso inclusi, non pregiudicano lo status o la sovranità su qualsiasi territorio, la delimitazione delle frontiere e dei confini internazionali e il nome di qualsiasi territorio, città o area.

(C) OCSE 2021

È possibile copiare, scaricare o stampare contenuti OCSE per uso personale e includere estratti di pubblicazioni OCSE, banche dati e prodotti multimediali nei propri documenti, presentazioni, blog, siti web e materiali didattici, a condizione che venga dato adeguato riconoscimento all'OCSE come fonte e proprietario dei diritti d'autore. Tutte le richieste di utilizzo pubblico o commerciale e di diritti di traduzione devono essere inviate a rights@oecd.org. 


\section{Riconoscimenti}

Questo paper è stato scritto da Mattia Corbetta del Centro OCSE di Trento per lo Sviluppo Locale, parte del Centro per I'Imprenditorialità, le PMI, le Regioni e le Città (CFE). L'autore ringrazia Karen Maguire e Alessandro Kandiah, della Divisione per l'Occupazione Locale, le Competenze e l'Innovazione sociale dell'OCSE, nonché Alessandra Proto e Wessel Vermeulen (Centro di Trento per lo Sviluppo Locale) per aver condiviso commenti dettagliati e preziosi suggerimenti. Inoltre, l'autore ringrazia Elisa Campestrin (Centro di Trento per lo Sviluppo Locale) per il contributo editoriale. L'OCSE ringrazia anche i delegati del Programma LEED di Australia, Canada, Giappone, Turchia e Regno Unito per i loro validi commenti. 


\section{Table of contents}

Riconoscimenti 3

$\begin{array}{ll}\text { Sintesi } & 6\end{array}$

Un nuovo approccio al telelavoro 9

Un cambiamento di prospettiva: da opportunità a necessità 9

Un cambiamento di scala: da una pratica di nicchia alla diffusione di massa 10

Un cambiamento di logica: da soluzione temporanea a permanente 10

Qual è la posta in gioco? Le principali questioni politiche 13

Un fenomeno complesso con impatti sociali diversificati 13

Come il telelavoro di massa potrebbe influire sui datori di lavoro e i dipendenti 14

$\begin{array}{ll}\text { Come il telelavoro di massa potrebbe influire sulle aree urbane e rurali } & 17\end{array}$

Come il telelavoro di massa potrebbe influire sulle imprese di diverse dimensioni $\quad 19$

La necessità di un approccio di policy lungimirante e basato sulle evidenze 20

Politiche per il telelavoro 23

Un insieme differenziato di politiche su più livelli per promuovere il telelavoro 23

Dalle raccomandazioni a breve termine ai piani a lungo termine per l'adozione del telelavoro $\quad 24$

Messa a punto di un quadro normativo per il telelavoro $\quad 25$

Piattaforme pubblico-private per il telelavoro e i servizi digitali 26

Sostegno non finanziario alle PMI per l'adozione del telelavoro: campagne informative e

formazione $\quad 27$

Sostegno finanziario alle PMI per l'adozione del telelavoro: sovvenzioni e altri incentivi 28

Politiche per l'attrazione dei lavoratori a distanza e dei lavoratori nel settore digitale $\quad 29$

Politiche a sostegno delle imprese home-based 31

Messaggi principali per i responsabili politici 33

L'impatto non neutrale del telelavoro su persone, territori e imprese 33

Nuove conoscenze sul telelavoro e percorsi futuri 34

Allegato A. Una rassegna delle politiche nazionali, regionali e locali per il telelavoro 40

$\begin{array}{ll}\text { Politiche nazionali per il telelavoro } & 40\end{array}$

$\begin{array}{ll}\text { Politiche regionali e locali per il telelavoro } & 49\end{array}$

$\begin{array}{ll}\text { Bibliografia } & 57\end{array}$ 
Tables

Tabella 1. Principali questioni politiche e opportunità e minacce più frequentemente associate al telelavoro (dipendenti, datori di lavoro, aziende, aree urbane, aree rurali, società)

Tabella 2. Tipologie di politiche per il telelavoro, per Paese, Regione e Città

\section{Boxes}

Box 1. Principi guida e raccomandazioni di policy per una transizione graduale verso un modello di telelavoro sostenibile per persone, territori e aziende 


\section{Sintesi}

Prima della pandemia di COVID-19 il telelavoro era concepito principalmente come un'opportunità di conciliazione tra lavoro e vita privata. Improvvisamente la pandemia lo ha trasformato in una soluzione urgente per ridurre il rischio di contagio e garantire la continuità dell'attività economica. Questa pratica è passata rapidamente dall'essere un'opzione occasionale concentrata principalmente in pochi Paesi, e in particolare nelle aree urbane, a una soluzione di massa adottata in tutta l'area OCSE e non solo. Secondo diversi sondaggi, numerose aziende a livello globale stanno valutando la possibilità di passare al telelavoro di massa su base permanente anche dopo la fine della crisi sanitaria dovuta al COVID-19.

Una transizione diffusa al telelavoro avrebbe impatti diversi su persone, luoghi e aziende. In particolare, questa trasformazione è destinata ad avere un effetto amplificato a livello locale. Mentre un declino delle città appare improbabile, il telelavoro di massa potrebbe promuovere la delocalizzazione di alcuni posti di lavoro lontano dalle grandi aree metropolitane e rappresentare un'opportunità di recupero per le regioni in ritardo di sviluppo. Questo potrebbe essere il caso delle città di piccole e medie dimensioni, nonché delle aree semi-dense e rurali dotate di internet ad alta velocità, che potrebbero attrarre quote crescenti di telelavoratori e trattenere un maggior numero di residenti, in un mondo in cui una quota crescente di posti di lavoro sarà accessibile da qualsiasi luogo. II telelavoro su vasta scala potrebbe anche condurre a cambiamenti sostanziali per i dipendenti e i datori di lavoro. Parte delle future prospettive per i lavoratori dipendenti di migliorare il proprio equilibrio tra lavoro e vita privata e per i datori di lavoro di trattenere i talenti potrebbe dipendere dalla rispettiva disponibilità a fare un maggiore utilizzo del telelavoro. In futuro, tuttavia, alcune disparità preesistenti (ad esempio tra lavoratori altamente e scarsamente qualificati, grandi aziende e PMI, ecc.) potrebbero aumentare sulla base delle diverse capacità di adattamento. Studi empirici forniscono prove contrastanti sull'impatto del telelavoro su una serie di aspetti chiave, tra cui la produttività del lavoro e il livello di soddisfazione dei dipendenti, implicando la necessità di un dialogo tra le diverse parti in causa e di un monitoraggio dei dati.

Dopo lo scoppio della pandemia i governi a tutti i livelli hanno adottato un insieme diversificato di politiche per affrontare le sfide poste dalla crisi e fornire risposte su misura per il telelavoro. In genere, i governi nazionali hanno adottato misure quadro per rendere praticabile il lavoro da casa. Mentre introducevano le misure di distanziamento sociale, hanno incoraggiato i datori di lavoro privati ad adottare questa modalità, adattando coerentemente il contesto normativo. Le opzioni andavano dall'introduzione di nuove norme, o l'adeguamento di regolamenti preesistenti, all'emanazione di linee guida o altri strumenti giuridici non vincolanti. Inoltre, diversi governi nazionali hanno creato piattaforme online per rendere accessibili a tutti i servizi digitali offerti dai grandi fornitori di tecnologie dell'informazione.

I governi regionali e locali, a loro volta, si sono concentrati sullo sviluppo delle risorse necessarie a livello locale per aumentare l'adozione del telelavoro. Molti di essi hanno riconosciuto la presenza di lacune nei processi di digitalizzazione, e hanno quindi dato priorità all'offerta di servizi informativi e di programmi di formazione ai cittadini e alle imprese. Al contrario, alcune comunità avanzate dal punto di vista dello sviluppo digitale hanno addirittura iniziato a progettare i primi esempi di piani a lungo termine per una più ampia diffusione del telelavoro su base permanente. Infine, vari governi, per lo più regionali, hanno ideato 
programmi di sostegno finanziario per favorire l'utilizzo del telelavoro da parte delle PMI, generalmente sovvenzionando investimenti in strumenti e competenze digitali.

Già prima dell'avvento della pandemia, Paesi e regioni di tutto il mondo avevano lanciato programmi per promuovere l'attrazione di lavoratori o imprenditori a distanza e del settore digitale, offrendo sovvenzioni, strutture di co-working, visti per l'avvio di start-up e altri strumenti. Altre autorità locali hanno istituito misure e incentivi per le imprese home-based, tra cui la modifica delle normative sui contratti di locazione e sulla tassazione dei beni immobili per promuovere questa modalità imprenditoriale. In questo contesto il telelavoro è stato inteso come uno strumento utile per contrastare l'invecchiamento della popolazione, prevenire la fuga dei cervelli e rendere più vivaci le comunità imprenditoriali locali. Misure di questo tipo, introdotte prima del COVID-19, potrebbero acquisire nuovo slancio attraverso il telelavoro di massa.

I seguenti principi guida potrebbero contribuire a promuovere una transizione graduale verso un utilizzo duraturo e su larga scala del telelavoro, favorendo un modello che sia sostenibile per i diversi gruppi sociali, i territori e le imprese:

a. Adottare un approccio olistico nell'ideazione delle politiche per il telelavoro. Una diffusione persistente e su vasta scala del telelavoro potrebbe influire profondamente sui mercati del lavoro locali nelle aree urbane e rurali, sui modelli residenziali e di mobilità, così come sulla società nel suo insieme. Questi impatti non sono ancora del tutto prevedibili. Una prospettiva multidisciplinare e a lungo termine nell'ideazione delle politiche contribuirebbe a declinare queste dinamiche trasformative a seconda delle opportunità di sviluppo locale.

b. Promuovere equità e inclusione tra i gruppi sociali, le imprese e i territori. Il telelavoro non è accessibile in modo uniforme per le diverse tipologie di lavoratori, territori (es. aree urbane e rurali) e imprese (es. PMl e grandi aziende) e ha effetti diversificati su ciascuno di essi. Le misure in materia di telelavoro potrebbero contribuire a creare condizioni di parità, a patto che si mettano in atto politiche sociali complementari.

c. Dare la priorità agli obiettivi di natura collettiva. Una diffusione su vasta scala del telelavoro potrebbe contribuire a ridurre il traffico urbano e le emissioni di $\mathrm{CO} 2$, favorire lo sviluppo rurale, promuovere l'uguaglianza di genere e perseguire altri obiettivi di sviluppo sostenibile. Gli interventi potrebbero dare la priorità a soluzioni vantaggiose per la collettività e attenuare le esternalità negative, come la drastica riduzione della domanda che interessa i negozi e i fornitori di servizi situati in prossimità degli uffici.

d. Fornire le condizioni quadro abilitanti a livello nazionale e locale. La presenza di un quadro regolamentare agile e di servizi pubblici locali efficaci (in settori quali l'assistenza all'infanzia e agli anziani), così come di reti internet ad alta velocità, competenze digitali e un ambiente online protetto sono requisiti necessari per rendere il telelavoro accessibile e sicuro per tutti.

e. Creare una nuova base di conoscenza. Date le caratteristiche inedite del telelavoro su vasta scala, è importante produrre nuovi dati e metriche per informare le decisioni politiche. Gli effetti dovrebbero essere monitorati nel tempo per evitare conclusioni non obiettive e tenere conto della molteplicità di fattori che influiscono sulle persone, le aziende e i territori. Aspetti quali la raccolta di dati e l'adattabilità alle condizioni di contesto dovrebbero essere tenuti in conto già dalla fase di ideazione delle politiche, prevedendo sistematicamente forme continue di monitoraggio e di valutazione.

Il documento si conclude con un'ampia gamma di raccomandazioni di policy a carattere preliminare per l'attuazione di ciascuno dei suddetti principi.

Questo lavoro si articola come segue: il primo capitolo contestualizza il tema del telelavoro, sottolineando come la pandemia di COVID-19 abbia modificato radicalmente l'approccio a un dibattito politico di lunga data. II secondo capitolo mostra che il telelavoro di massa è un fenomeno complesso che interessa molte aree di policy e che ha effetti diversificati su gruppi sociali, territori e aziende. II terzo capitolo evidenzia 
8

come durante la pandemia di COVID-19 tutti i livelli di governo abbiano agito per sostenere il telelavoro, e fornisce una classificazione delle politiche adottate. II quarto capitolo offre spunti per i governi nazionali e regionali. Infine, l'Allegato A presenta una rassegna di pratiche di policy sul telelavoro tratte da Paesi, Regioni e Città dell'area OCSE e non solo. 


\section{Un nuovo approccio al telelavoro}

\section{Un cambiamento di prospettiva: da opportunità a necessità}

La pandemia di COVID-19 ha modificato radicalmente la prospettiva che tradizionalmente caratterizzava il dibattito sul telelavoro. ${ }^{1}$ In passato, la motivazione principale alla base dei programmi aziendali di lavoro a distanza era l'opportunità di attrarre e trattenere i talenti. L'epidemia ha trasformato il telelavoro in una soluzione di salute pubblica, spinta dalla necessità di ridurre il rischio di contagio, garantendo al contempo una continuità nell'attività economica.

In passato, il telelavoro era stato concepito per lo più come uno strumento di conciliazione tra lavoro e vita privata. Un rapporto dell'OCSE del 2016 presentava questa pratica come un esempio di modalità di lavoro flessibile in grado di migliorare l'equilibrio tra lavoro e vita privata dei dipendenti e di rispondere anche alle esigenze della parte datoriale. Lo studio suggeriva che una maggiore diffusione delle tecnologie dell'informazione e della comunicazione avrebbe potuto aumentare l'utilizzo del telelavoro, ma metteva anche in guardia contro il rischio di un'attenuazione della linea di demarcazione tra lavoro e vita familiare. Inoltre, la diffusione delle tecnologie informatiche avrebbe potuto influire sui diversi lavori e le mansioni in modi differenti, ampliando piuttosto che riducendo le disuguaglianze. Nel complesso, la diffusione di modalità di lavoro flessibili in tutte le occupazioni e le aziende avrebbe richiesto creatività nel ripensare $\mathrm{i}$ modelli esistenti di organizzazione del lavoro e sulle pratiche gestionali (OECD, 2016[1]).

Circostanze radicalmente mutate gettano nuova luce sulle motivazioni tradizionali a favore del telelavoro. Una nota dell'OCSE del 2020 sull'occupazione nel contesto della pandemia di COVID-19 evidenzia che molti Paesi hanno adottato misure per limitare gli spostamenti quotidiani da e verso l'azienda e l'interazione fisica sul posto di lavoro, al fine di ridurre il rischio di contrarre e diffondere il coronavirus. In questo contesto il telelavoro rappresenta una soluzione chiave tra le varie opzioni a disposizione dalla politica per ridurre l'esposizione dei lavoratori al COVID-19 e aiutare le aziende a superare la crisi (OECD, 2020a[2]).

Poiché il telelavoro è diventato di attualità durante la pandemia, vi è una crescente attenzione in merito agli impatti diversificati e agli effetti incerti sulla produttività e altri indicatori indotti dalla trasformazione in atto. Le politiche pubbliche possono assumere un ruolo decisivo nello sfruttare al massimo i vantaggi derivanti da un utilizzo più diffuso del telelavoro in termini di produttività e di benessere. Tra le varie opzioni, i governi potrebbero incentivare investimenti nelle dotazioni strumentali e nelle competenze manageriali delle imprese e dei lavoratori necessari per il telelavoro, nonché affrontare le potenziali problematiche per il benessere dei lavoratori e per l'innovazione a lungo termine associati al ridimensionamento degli spazi di lavoro (OECD, 2020 $\left.\mathrm{b}_{[3]}\right)$.

\footnotetext{
${ }^{1}$ L'OCSE definisce il telelavoro come una forma di lavoro mobile facilitato dalle tecnologie dell'informazione e della comunicazione che si svolge a casa o in un altro luogo al di fuori del tradizionale posto di lavoro (OECD, 2019, p. 80[6]).
} 


\section{Un cambiamento di scala: da una pratica di nicchia alla diffusione di massa}

Tale cambiamento di prospettiva è andato di pari passo con la crescente importanza di questa pratica. Da opzione diffusa in modo disomogeneo e ancora concentrata principalmente in pochi Paesi, con l'avvento della pandemia di COVID-19 il telelavoro ha rapidamente assurto a soluzione adottata su larga scala in tutto il mondo. Le differenze nelle normative per il contenimento del virus e le forti variazioni nei loro impatti tra Paesi e settori sono di ostacolo all'elaborazione di stime precise sull'attuale diffusione del telelavoro a livello globale. Tuttavia, è opinione comune che la pandemia di COVID-19 abbia comportato, tra le altre cose, una sperimentazione del telelavoro su larga scala, e che il numero di telelavoratori sia aumentato significativamente negli ultimi mesi. Sebbene le evidenze suggeriscano che i tassi di utilizzo del telelavoro stessero crescendo anche prima della pandemia, quest'ultima ha accelerato enormemente tale dinamica.

In Europa, i dati mostrano una crescita sensibile del telelavoro. Già al 24 marzo 2020, circa un mese dopo che la pandemia era diventata di dominio pubblico, 21 dei 31 Paesi UE/SEE avevano imposto la chiusura dei luoghi di lavoro o introdotto il telelavoro (European Centre for Disease Prevention and Control, 2020[4]). Secondo l'Indagine Europea sulle Condizioni di Lavoro del 2015, la percentuale di dipendenti che avevano lavorato da casa almeno una volta nell'ultimo anno andava dal $10 \%$ registrato in Slovacchia, Italia e Bulgaria al 50\% di Svezia e Danimarca. Nei Paesi nordici, i lavoratori dipendenti (soprattutto quelli con figli) avevano il doppio delle probabilità di lavorare da casa rispetto alla media europea. Circa il $9 \%$ dei lavoratori UE aveva utilizzato le tecnologie dell'informazione e della comunicazione al di fuori dei locali del datore di lavoro: il $2 \%$ aveva telelavorato principalmente da casa mentre il $7 \%$ era costituito da lavoratori mobili che utilizzavano in misura prevalente dispositivi digitali (Eurofound, 2015[5]). Solo tre anni dopo, ma ancora prima dello scoppio dell'epidemia, l'OCSE stimava che la percentuale di persone che utilizzavano strumenti digitali al lavoro e che avevano telelavorato da casa almeno una volta alla settimana nell'UE era cresciuta fino a un quarto della forza lavoro complessiva (OECD, 2019[6]). Stime più recenti (aprile 2020) suggeriscono che quasi il $40 \%$ dei dipendenti nell'UE ha iniziato a telelavorare a tempo pieno a seguito della pandemia (Eurofound, 2020[7]).

Un tasso complessivo simile (39\%) è stato registrato per diversi i Paesi dell'area OCSE nella primavera del 2020 (OECD, 2020c, p. 12[8]), anche se il ritmo di crescita nel ricorso a questa pratica è variabile. All'inizio di aprile 2020, fino alla metà dei lavoratori americani lavorava da casa - più del doppio rispetto a quelli che lavoravano da casa, almeno occasionalmente, nel 2017-18 (Brookings, 2020[9]). In Francia, la prevalenza del telelavoro appare più bassa, ma il suo aumento rispetto al periodo precedente alla pandemia è più pronunciato: un'indagine condotta da un'organizzazione indipendente il 19 maggio 2020 ha stimato che il $39 \%$ dei dipendenti francesi $(7$ milioni di lavoratori) ha telelavorato a partire da metà marzo (ODOXA, 2020 $\mathrm{a}_{[10]}$ ), mentre secondo il Ministero del Lavoro il tasso di dipendenti che lavoravano da casa almeno una volta alla settimana era di appena il 3\% nel 2017 (DARES, 2019 [11]).

Diversi fattori sono alla base di queste variazioni tra Paesi. Le differenze nella composizione settoriale, la distribuzione dell'occupazione per dimensioni d'impresa, il tasso di lavoro autonomo e le competenze digitali dei lavoratori sono tra i principali fattori che spiegano una diversa prevalenza del telelavoro nei Paesi UE (JRC, 2020[12]). La qualità dell'infrastruttura digitale è un altro fattore alla base delle differenze tra regioni all'interno dei Paesi.

Non da ultimo, va sottolineato che le misure di contenimento non riguardano solo la prestazione di lavoro in sé, ma anche ambiti correlati. L'istruzione e la formazione professionale (ILO, 2020a[13]) e la formazione sul posto di lavoro (McKinsey, 2020a[14]) possono essere citate come esempio.

\section{Un cambiamento di logica: da soluzione temporanea a permanente}

Una transizione diffusa verso il telelavoro avrà importanti conseguenze sul mondo del lavoro e sulla produzione anche dopo la pandemia. Un'"economia del distanziamento sociale", in cui il lavoro a distanza 
e riunioni, commercio e pubblicità online rappresentano la normalità, e le attività a distanza costituiscono una prassi molto più abituale rispetto al passato, potrebbe permanere per qualche tempo (Criteo, 2020[15]).

Molti posti di lavoro nei Paesi OCSE sono effettivamente compatibili con il telelavoro. Studi recenti indicano che il $56 \%$ della forza lavoro statunitense ha un'occupazione compatibile (almeno in parte) con il telelavoro, e che il $25-30 \%$ della forza lavoro lavorerà probabilmente da casa per più giorni alla settimana fino alla fine del 2021.

Diversi fattori concomitanti contribuiranno a un'impennata del telelavoro, come l'aumento della domanda di lavoro da casa da parte dei dipendenti. Già prima della crisi, alcuni sondaggi ripetuti nel tempo hanno mostrato che l' $80 \%$ dei dipendenti americani vuole lavorare da casa almeno per una parte del tempo, e più di un terzo si ridurrebbe lo stipendio in cambio di questa possibilità. Queste fonti mostrano anche che gli stessi manager che hanno lavorato da casa sono più propensi ad acconsentire a tale istanza a beneficio del personale. Inoltre, promuovendo il lavoro da casa, ci sarà una maggiore consapevolezza riguardo alle opportunità di riduzione dei costi. Un tipico datore di lavoro può risparmiare circa 11000 dollari all'anno per ogni persona che lavora a distanza per la metà del tempo, grazie alla riduzione degli spazi per uffici e dei costi dei viaggi d'affari. I dipendenti possono risparmiare tra i 2500 e i 4000 dollari all'anno (lavorando a distanza la metà del tempo) e anche di più se sono nelle condizioni di traslocare in un'area residenziale meno costosa e lavorare a distanza a tempo pieno (Global Workplace Analytics, 2020[16]).

Benché attraverso metodologie e calcoli differenti, altri studi sugli Stati Uniti confermano già dall'inizio della pandemia l'esistenza di piani a più lungo termine per l'utilizzo del telelavoro. Un'indagine su 317 direttori e leader finanziari condotta da una società di ricerca e consulenza globale il 30 marzo 2020 ha rivelato che, dopo la pandemia, il 74\% punta a spostare almeno il $5 \%$ della forza lavoro precedentemente presente sul posto di lavoro verso una modalità di lavoro a distanza permanente. Inoltre, quasi un quarto degli intervistati ha dichiarato di avere intenzione di spostare almeno il $20 \%$ dei propri dipendenti in loco verso modalità di lavoro a distanza permanente (Gartner, 2020[17]).

Tuttavia, le previsioni che indicano una più ampia adozione del telelavoro non si limitano ai Paesi OCSE. Secondo una recente indagine condotta dalla società di servizi immobiliari Cushman \& Wakefield con oltre 2000 proprietari e locatari di uffici in Cina, circa il 21\% delle imprese intervistate cambierà in futuro il proprio modo di lavorare e adotterà una piattaforma di lavoro da casa/a distanza per i dipendenti del proprio ufficio, e il $50 \%$ è ancora indeciso. Inoltre, è probabile che l' $81 \%$ delle imprese intervistate in Cina investa in software e dispositivi di tecnologie informatiche più intelligenti, come software e dispositivi cloud per le conferenze. II loro obiettivo è quello di migliorare l'esperienza di lavoro da casa o a distanza per i loro dipendenti e di aumentarne il coinvolgimento e la produttività del lavoro (Cushman \& Wakefield, 2020[18]).

In definitiva, è in corso un cambiamento nella natura stessa del telelavoro e sono osservabili tre approcci distinti, ognuno con implicazioni diverse. II "lavoro da casa" replica il più possibile le condizioni di lavoro dell'ufficio, con regole ben definite per quanto riguarda gli orari, i mezzi e persino gli spazi di lavoro. Questa concezione del telelavoro prevaleva prima del COVID-19. Al contrario, il "lavoro basato sugli obiettivi" non prevede l'applicazione di un orario di lavoro: i dipendenti, in modo simile ai liberi professionisti, hanno compiti da svolgere nei modi che ritengono più adatti e con i mezzi che hanno a disposizione. Questo è ciò che è accaduto spesso per necessità durante l'isolamento durante la pandemia. In terzo luogo, il "lavoro misto" (noto come "smart working" in alcuni Paesi, tra cui l'Italia) unisce le modalità del lavoro da casa e in ufficio, e comporta incontri con altri colleghi e la condivisione delle scrivanie nei giorni concordati. Si tratta di un approccio flessibile che è molto probabile susciterà grande interesse da parte delle aziende nella fase post-emergenziale (Global Workplace Analytics, 2020[16]).

Nel lungo periodo, quando l'adozione del telelavoro non sarà più determinata dalla necessità di affrontare una crisi sanitaria, i settori, le aziende e i lavoratori avranno, con ogni probabilità, approcci diversi alla "nuova normalită" per poter massimizzare la produttività e l'innovazione. Sebbene alcune tipologie di attività traggono maggiori benefici dalla presenza fisica (ad esempio la formazione del personale e le sessioni di brainstorming), le aziende dovranno comunque incoraggiare il telelavoro o saranno superate 
da quelle che lo faranno. Questo è coerente con quanto riscontrato a proposito della tendenza delle aziende verso l'adozione di un modello ibrido, che implicherebbe un significativo, anche se non drastico, aumento del telelavoro nel lungo termine. Un recente studio suggerisce che, rispetto alla situazione preesistente, nel mondo post-pandemico un decimo dei giorni di lavoro (un quinto per chi lavora in ufficio) si trasferirà dall'azienda alle abitazioni (Barrero et al., 2020[19]). Le prime indicazioni sono quindi che nella maggior parte dei casi il lavoro da casa aumenterà, ma non costituirà ancora una pratica prevalente e sarà adattato alle esigenze dei lavoratori e delle imprese in modo che abbia risvolti positivi per la produttività.

Qualunque sia la forma che assumerà il telelavoro, governare lo sviluppo economico e occupazionale a livello locale ai tempi del lavoro a distanza richiede una piena comprensione della posta in palio da parte di tutti i soggetti interessati. Pertanto, la prossima sezione cercherà di evidenziare le potenziali implicazioni del telelavoro di massa su persone, territori e imprese. 


\section{Qual è la posta in gioco? Le principali questioni politiche}

\section{Un fenomeno complesso con impatti sociali diversificati}

L'impennata nell'utilizzo del telelavoro durante la pandemia di COVID-19 ha contribuito a garantire la sicurezza dei lavoratori e la continuità delle attività economiche. Tuttavia, questa modalità di lavoro pone numerose sfide sia ai datori di lavoro che ai dipendenti. Se l'adozione diffusa del telelavoro dovesse permanere oltre la pandemia, tali cambiamenti potrebbero avere forti ripercussioni sui mercati del lavoro e sui diversi settori dell'economia a livello locale. La propensione all'uso massiccio del telelavoro determinerà le possibilità future dei dipendenti di migliorare il proprio equilibrio tra lavoro e vita privata, dei datori di lavoro di trattenere i talenti, delle regioni di attrarre posti di lavoro di qualità e delle PMI di rimanere competitive. II telelavoro ha acquisito nuovo slancio anche nel dibattito politico di diversi Paesi, in particolare per quanto riguarda i diritti dei lavoratori. Gli argomenti al centro oggetto di dibattito negli ultimi anni sono tornati di attualità in tutto il mondo, coinvolgendo diverse discipline e attori sociali.

In Germania, la proposta di introdurre un diritto al telelavoro era stata parte del dibattito politico molto prima della pandemia di COVID-19. Nel 2019, un'iniziativa di consultazione con più soggetti interessati, nota come "Dialogo sul Futuro", ha suscitato l'attenzione dell'opinione pubblica in proposito. Un sondaggio che ha mostrato come un terzo dei dipendenti fosse disposto a sperimentare il telelavoro ha acceso il dibattito (BMAS, 2019[20]). Mentre una parte dello spettro politico ha promosso l'introduzione di un "diritto al telelavoro" in contrapposizione alla "cultura della presenza sul posto di lavoro" (con una minoranza che sostiene persino il "diritto alla disconnessione"), altri hanno sostenuto che accordi più flessibili sarebbero stati più efficaci rispetto all'introduzione di nuove leggi per migliorare l'equilibrio tra lavoro e vita privata. (Der Spiegel, 2019[21]). Negli ultimi mesi, i sostenitori del diritto al telelavoro non hanno esitato a riprendere la questione e a portarla in cima all'agenda politica (Der Spiegel, 2020a[22]).

La pandemia sta rinnovando la giurisprudenza in materia di telelavoro, anche in relazione a casi precedenti alla pandemia. Nell'aprile del 2020 la Corte Suprema Federale Svizzera ha emanato una sentenza a favore della richiesta di un dipendente di ottenere il risarcimento dei costi associati al telelavoro da parte del datore di lavoro, sulla base del principio secondo cui i costi di produzione devono essere sostenuti dal datore di lavoro. Nel caso in questione, che risale al 2019, la dipendente ha chiesto il riconoscimento delle spese derivanti dall'aver utilizzato parte del suo appartamento per lavorare, in quanto non disponeva di una propria postazione di lavoro presso la sede dell'azienda. Secondo gli esperti locali in materia di lavoro, la sentenza è suscettibile di generare un nuovo dibattito e, possibilmente, giurisprudenza in materia di diritti dei lavoratori, in quanto durante la pandemia un gran numero di dipendenti ha dovuto trasformare una parte delle proprie abitazioni in luoghi di lavoro, assumendosi parte dei costi di produzione (LaRegione, 2020[23]).

Anche le parti sociali hanno avuto un ruolo centrale nel dibattito. In Italia, secondo le stime del principale sindacato del Paese, i telelavoratori sono passati da 0,5 milioni a 8 milioni di persone durante la pandemia di COVID-19. Nel maggio 2020, lo stesso sindacato ha effettuato la prima indagine nazionale in materia. Anche se il $60 \%$ degli attuali telelavoratori intendeva continuare con questa modalità dopo la pandemia, il $31 \%$ pensava di non avere le competenze necessarie, la metà pensava di non avere abbastanza spazio 
dedicato presso la propria abitazione, e il $65 \%$ delle donne ha riscontrato un aumento del carico di lavoro domestico (Cgil/Fondazione di Vittorio, 2020[24]). Solo poche settimane prima, la maggiore associazione datoriale a livello nazionale aveva segnalato una maggiore propensione da parte delle aziende rispetto al passato ad adottare il telelavoro (Confindustria, 2020[25]).

Altri attori sociali ed economici di tutto il mondo stanno esaminando da vicino le attuali tendenze del telelavoro, riflettendo la varietà degli interessi coinvolti. Ad esempio, mentre le grandi società immobiliari si stanno interrogando sul futuro dei grandi edifici adibiti a uffici nelle città finanziarie globali (Der Spiegel, $2020 b_{[26]}$, architetti e urbanisti prevedono il grande ritorno delle città storiche in aree remote e spopolate (The Times, 2020[27]).

Tutto ciò è indicativo della una moltitudine di prospettive, opportunità e sfide connaturate in un'eventuale transizione di massa verso il telelavoro, così come dell'entità del suo potenziale impatto socio-economico. Una ripartizione delle diverse questioni in gioco tra i diversi gruppi sociali (datori di lavoro e dipendenti), i territori (mercati del lavoro locali) e le imprese (comprese le PMI) evidenzia l'ampiezza del cambiamento in corso e delle sue implicazioni per i responsabili politici. La Tabella 1 concluderà questa sezione con una sintesi delle principali questioni emerse.

\section{Come il telelavoro di massa potrebbe influire sui datori di lavoro e i dipendenti}

Mentre i telelavoratori devono affrontare la sfida di bilanciare efficacemente il lavoro e la vita privata senza pregiudicare la produttività e la partecipazione al lavoro di squadra, i loro datori di lavoro devono confrontarsi con la necessità di fornire l'infrastruttura digitale, la formazione e garantire un ambiente di lavoro virtuale sicuro. Una prima domanda fondamentale è quindi se i benefici di una transizione di massa verso il telelavoro supererebbero i costi. Gli studi precedenti forniscono evidenze contrastanti su una serie di aspetti cruciali, tra cui le variazioni di produttività e i miglioramenti nella conciliazione vita-lavoro dei dipendenti. I risultati spesso dipendono dal tipo di occupazione, dal settore e persino dal lavoratore. Diversi gruppi di lavoratori sono esposti alle opportunità e alle minacce derivanti dal telelavoro in modi differenti: lavoratori a tempo indeterminato e temporanei, lavoratori dipendenti e autonomi, genitori e non, maschi e femmine, senior e junior, redditi/competenze elevati e ridotti, residenti e transfrontalieri, locali e immigrati, ecc. - ciascuno di questi gruppi ha una propria prospettiva sulla questione. Ciò evidenzia la necessità di un approccio di policy versatile, in grado di discernere l'impatto diversificato del telelavoro sui diversi settori, sulle tipologie di lavoro e sulle mansioni.

II telelavoro e il lavoro mobile sono comunemente associati a una serie di effetti positivi, come una maggiore autonomia rispetto all'orario di lavoro che comporta una maggiore flessibilità in termini di organizzazione del tempo dedicato al lavoro, e una riduzione di quello dedicato agli spostamenti da e verso il posto di lavoro che si traduce a sua volta in un migliore equilibrio complessivo tra lavoro e vita privata e una maggiore produttività. Allo stesso modo, gli svantaggi tipicamente riconosciuti includono la tendenza a lavorare più a lungo, e una sovrapposizione tra lavoro e vita privata - che può portare ad alti livelli di stress. Si può tracciare una distinzione tra i telelavoratori da casa, che sembrano godere di un migliore equilibrio tra lavoro e vita privata, e i lavoratori "ad elevata mobilità" (altri lavoratori che presentano un alto livello di utilizzo delle tecnologie informatiche), che sono soggetti a rischi maggiori per la salute e il benessere (Messenger et al., 2017 [28]). II modo e la misura in cui l'isolamento professionale potenzialmente derivante dal telelavoro influenza le prestazioni lavorative e i cambiamenti di personale è un altro argomento dibattuto in letteratura. (Golden, Veiga and Dino, 2008[29]).

Riscontri recenti confermano la tendenza ad aumentare il numero di ore lavorate. Le statistiche raccolte durante il lockdown hanno mostrato non solo un chiaro picco nell'utilizzo delle VPN aziendali, ma anche che le persone lavorano molto più a lungo del solito. Gli Stati Uniti hanno aumentato la giornata lavorativa media di quasi il 40\%, aggiungendo tre ore in più, il balzo più significativo a livello globale. Regno Unito, Francia, Canada e Spagna hanno registrato un aumento di due ore. Ciò è dovuto, almeno in parte, al fatto 
che i dipendenti hanno iniziato a lavorare prima del solito, poiché non hanno avuto bisogno di recarsi sul posto di lavoro, ma hanno chiuso la giornata lavorativa alla solita ora (Business Facilities, 2020 [30]). In parte gli aumenti nell'uso di VPN possono anche essere legati alla necessità di occuparsi contemporaneamente di diverse incombenze e quindi è necessario più tempo per svolgere gli stessi compiti, soprattutto se si considerano gli oneri di assistenza all'infanzia derivanti dalla chiusura delle scuole e degli asili.

Per quanto riguarda la produttività, gli studi empirici forniscono risultati divergenti a seconda del tipo di lavoro e delle mansioni specifiche prese in considerazione. I risultati di uno studio del 2012 suggeriscono che il telelavoro può avere implicazioni positive sulla produttività per quanto riguarda le attività creative, ma negative rispetto a quelle monotone e ripetitive (Dutcher G., 2012[31]). Secondo un'indagine condotta in Giappone durante la pandemia, i lavoratori indicano che sono, in media, meno produttivi a casa che in ufficio. Mentre alcune delle ragioni alla base di questo risultato, come la mancanza di familiarità con $\mathrm{i}$ software di accesso remoto, si attenueranno nel tempo, altri fattori, come la mancanza di interazione faccia a faccia con i colleghi, suggeriscono che permarrà un divario di produttività (VOX EU, 2020[32]). Tuttavia, mentre un rapporto WISERD propone una gamma più equilibrata di punti di vista sulla produttività (Felstead and Reuschke, 2020[33]) altri studi hanno riscontrato che la collaborazione (in termini di numero di incontri) è aumentata durante la pandemia (Defilippis et al., 2020[34]), senza dimenticare come uno studio pre-COVID, spesso citato, sui call center cinesi ha riscontrato impatti positivi del lavoro da casa sulla produttività (Bloom et al., 2014[35]). Inoltre, il Rapporto al Congresso sul Telelavoro del 2018 suggerisce che il telelavoro implichi un risparmio significativo in termini di riduzione delle assenze dei dipendenti (US Office of Personnel Management, 2017[36]).

Recenti lavori dell'OCSE forniscono un'analisi approfondita della letteratura esistente sul nesso tra telelavoro e produttività e sugli effetti positivi e negativi. Nel complesso, affinché la produttività a livello aziendale cresca con il telelavoro è fondamentale che la soddisfazione dei lavoratori aumenti a sufficienza per compensare gli effetti potenzialmente negativi sulla comunicazione, sui flussi di conoscenza e sulla supervisione manageriale. L'impatto relativo di ciascuno di questi fattori, a sua volta, dipenderà probabilmente dall'intensità del telelavoro. L'effetto negativo dovuto alla mancanza di interazioni personali cresce presumibilmente di pari passo con l'intensità del telelavoro, diminuendo man mano le opportunità di comunicare di persona. La soddisfazione del lavoratore migliora con bassi livelli di telelavoro, ma può soffrire di un "eccessivo" telelavoro, ad esempio a causa della solitudine o della sovrapposizione tra vita privata e professionale. L'efficienza del lavoratore migliora quindi con bassi livelli di telelavoro, ma diminuisce con il telelavoro eccessivo, il che implica un "punto ottimale" in cui l'efficienza del lavoratore e quindi la produttività - sono massimizzate con livelli intermedi di telelavoro (OECD, 2020b $\mathrm{b}_{[3]}$ ). Secondo diversi osservatori, al di là degli effetti interni all'azienda, un passaggio in massa al telelavoro rischia di esacerbare le disparità tra i vari tipi di lavoratori nei diversi settori e nelle regioni. Nel 2017, un'indagine promossa dal Ministero del Lavoro francese ha mostrato che il 60,6\% dei telelavoratori regolari erano dirigenti esecutivi (quadri), che rappresentavano solo il 16,9\% di tutti i dipendenti. Mentre l' $11,1 \%$ dei dirigenti e il 3,2\% delle professioni intermedie hanno telelavorato almeno un giorno alla settimana, la pratica è ancora marginale tra i dipendenti $(1,4 \%)$ e quasi inesistente tra gli operai $(0,2 \%)$ (DARES, $\left.2019_{[11]}\right)$. Nel maggio 2020, una nuova indagine di un'agenzia di sondaggi francese ha confermato la presenza di differenze significative tra i vari gruppi: tra i telelavoratori, l' $89 \%$ dei dirigenti, il $54 \%$ dei "quadri intermedi", il 26\% dei dipendenti e solo il 3\% dei lavoratori manuali (ODOXA, 2020a[10]). Un'indagine condotta nell'aprile 2020 dalla stessa agenzia di sondaggi ha evidenziato il problema dei differenziali regionali: il $41 \%$ della forza lavoro ha telelavorato nell'Île-de-France, contro l'11\% in Normandia (ODOXA, $\left.2020 b_{[37]}\right)$.

Ci sono solide evidenze riguardo al fatto che i lavoratori a più alto reddito sono stati in grado di ridurre $\mathrm{i}$ loro spostamenti molto di più rispetto a quelli a basso reddito. Secondo i dati sulla localizzazione degli smartphone negli Stati Uniti, i lavoratori a basso reddito erano più propensi a continuare a spostarsi quotidianamente durante il lockdown, mentre i lavoratori più ricchi avevano maggiori possibilità di rimanere a casa e di limitare la loro esposizione al coronavirus. Sebbene le persone di tutti i gruppi di reddito si 
siano spostate meno rispetto a prima della crisi, i lavoratori a più alto reddito hanno limitato maggiormente i loro spostamenti, soprattutto durante la settimana lavorativa. In quasi tutti gli stati, hanno iniziato a farlo giorni prima rispetto ai lavoratori con reddito basso, beneficiando del distanziamento sociale in una fase precedente del contagio. II differenziale era particolarmente elevato nelle aree metropolitane con la maggiore disparità pre-COVID tra i residenti più ricchi e quelli più poveri (Valentino-DeVries J. et al., $2020_{[38]}$ ). Altre evidenze recenti vanno nella stessa direzione: i lavoratori a più alto reddito hanno molte più probabilità di lavorare da casa durante la pandemia e molto meno probabilità di non poter lavorare affatto rispetto ai lavoratori a basso reddito (V. Reeves R. Rothwell J., 2020[39]).

Mentre è probabile che molti datori di lavoro continueranno a permettere al personale di lavorare da casa in futuro grazie ai risparmi sui costi, diversi osservatori, come la Confederation of British Industry, hanno sottolineato che il telelavoro è più facile per i lavoratori anziani con case più grandi e un maggiore capitale sociale accumulato sul posto di lavoro, mentre il personale più giovane potrebbe voler passare più tempo in ufficio (O'Connor Sarah, 2020[40]). Un ulteriore divario tra gruppi di lavoratori può dipendere dalle competenze. Recenti lavori dell'OCSE, che si basano sui dati dell'indagine OCSE sulle competenze degli adulti, mostrano che, nei Paesi OCSE, i lavoratori con livelli di competenze più elevati hanno maggiori probabilità di telelavorare. Mentre nell'area OCSE il 30\% dei lavoratori potrebbe telelavorare, la probabilità diminuisce per i lavoratori senza istruzione terziaria e con livelli più bassi di capacità computazionali e di alfabetizzazione. I risultati sollevano importanti interrogativi riguardo alla misura in cui la pandemia potrebbe esacerbare le disuguaglianze esistenti sul mercato del lavoro, e queste disuguaglianze potrebbero peggiorare ulteriormente a causa dell'intensificarsi dell'adozione di tecnologie all'indomani della pandemia (Espinoza and Reznikova, 2020[41]).

Nell'analizzare le ripercussioni del telelavoro di massa si dovrebbe distinguere tra il telelavoro come scelta, cioè come parte di un'organizzazione globale del lavoro, e il lavoro da casa imposto a causa di un'emergenza sanitaria. Mentre alcuni lavoratori potrebbero trovare vantaggioso sovrapporre la vita familiare con l'orario di lavoro, altri potrebbero volerli separare il più possibile, anche se sono impiegati in professioni che si prestano al telelavoro, suggerendo la presenza di approcci differenti per gestire $\mathrm{i}$ potenziali conflitti tra vita professionale e vita privata (Nafishah, Shafiz Affendi Mohd and Wan Rozaini Sheik, 2009[42]). Tuttavia non si sa se, e in che misura, il telelavoro sarà ancora una questione di scelta dopo il COVID-19. Per questo e per molti altri motivi, il monitoraggio sarà cruciale in uno scenario postlockdown.

Il telelavoro di massa solleva una serie di preoccupazioni dal punto di vista sociale, anche se molte delle osservazioni attuali derivano dalla situazione insolita legata alla pandemia. Ad esempio, la questione del telelavoro come scelta è particolarmente rilevante dal punto di vista dell'uguaglianza di genere, poiché vi sono segnali che l'aumento dei gli oneri legati all'accudimento dei bambini e delle responsabilità di assistenza in generale hanno effetti negativi sulla produttività anche sulle donne più istruite (ad esempio in ambito accademico) (Fazackerley, 2020[43]). Inoltre gli studi dimostrano che le misure di contenimento adottate in tutto il mondo per contrastare il coronavirus hanno aumentato i rischi associati alla violenza domestica, soprattutto per le donne, i bambini e le persone LGBTQ+ (CFR, 2020[44]). Infine, alla luce dell'impatto di un maggiore isolamento dalla società potenzialmente derivante dal telelavoro e del correlato aumento delle situazioni di solitudine, è importante valutare non solo i costi per la salute mentale, ma anche quelli per la salute fisica. La ricerca suggerisce un impatto emotivo negativo del telelavoro, in particolare in termini di emozioni quali solitudine, irritabilità, preoccupazione e senso di colpa, e che i telelavoratori sperimentano in modo significativo più sintomi legati alla salute mentale, quali lo stress, rispetto ai lavoratori in ufficio e, seppur lievemente, più sintomi legati alla salute fisica (Mann and Holdsworth, 2003[45]). 


\section{Come il telelavoro di massa potrebbe influire sulle aree urbane e rurali}

Non tutti i territori sono pronti ad adattarsi a un brusco cambiamento dei modelli di lavoro senza riscontrare difficoltà. Le regioni mostrano quote diverse di posti di lavoro che si prestano al telelavoro, a seconda delle competenze locali, delle infrastrutture digitali e della composizione della loro base industriale. Mentre il divario digitale interregionale può esacerbare le disparità preesistenti tra i mercati del lavoro locali, le regioni attualmente in ritardo, come le zone rurali, interne o montane, così come le città di piccole e medie dimensioni, potrebbero diventare destinazioni attraenti per i telelavoratori, offrendo standard di vita più elevati a costi più bassi. La capacità di tali luoghi di attrarre nuovi residenti dipenderà dalle risorse locali (non tutte influenzabili dalle politiche pubbliche, ad esempio le risorse ambientali come la presenza di aree naturalistiche, la qualità dell'aria e le condizioni atmosferiche) e dalle infrastrutture, insieme ad altri elementi di attrattività come il costo della vita, il collegamento con i centri di maggiori dimensioni e la capacità di raggiungere una massa critica nella fornitura di servizi. Tuttavia l'espansione del telelavoro potrebbe innescare una maggiore delocalizzazione di alcune attività, contribuendo a un rimescolamento nella localizzazione dei posti di lavoro sia all'interno che tra Paesi. Una serie di studi pubblicati durante i primi mesi della pandemia di COVID-19 cerca di esplorare la dimensione territoriale del telelavoro di massa.

L'aumento a breve termine del telelavoro potrebbe innescare una più ampia accettazione di questa forma di lavoro nel lungo periodo, con effetti differenti a seconda delle regioni. Un picco dell'adozione del telelavoro potrebbe contribuire ad un certo decentramento dei posti di lavoro lontano dalle grandi aree metropolitane o ai loro margini esterni, poiché i lavoratori sarebbero più liberi di trasferirsi dove il costo della vita è più basso o la qualità della vita è più alta. Tali spostamenti potrebbero contribuire a mitigare l'aumento dei prezzi degli alloggi nelle grandi città, così come la congestione del traffico regolare dei pendolari, aprendo opportunità per una crescita delle comunità rurali o per le aree metropolitane più piccole di attrarre residenti (OECD, $\left.2020 \mathrm{~d}_{[46]}\right)$. Tuttavia questa tendenza potrebbe anche avere effetti negativi sulle città. Ad esempio, i proprietari di negozi che dipendono dagli uffici e dai pendolari, il cui reddito è stato cancellato durante la pandemia (e sono soggetti agli stessi alti affitti urbani dei dipendenti) potrebbero trovarsi in una posizione di grande svantaggio. La Francia sta valutando la possibilità di sostenere su larga scala i piccoli negozi di quartiere per attenuare queste ripercussioni (Livini, 2020[47]).

In una certa misura, tali spostamenti sono in corso di pianificazione o stanno già avvenendo. In un recente studio incentrato sul Regno Unito, il $15 \%$ delle persone intervistate ha dichiarato che stava pensando di trasferirsi a causa del lockdown, mentre un terzo (34\%) ha dichiarato di pensare in modo diverso alla propria abitazione a causa dell'epidemia di COVID-19, in particolare per quanto riguarda l'importanza di un giardino e la necessità di più spazio interno per il lavoro da casa (Nationwide, $2020_{[48]}$ ). Allo stesso tempo negli Stati Uniti vi sono già evidenze di persone che si muovono verso aree a bassa densità di popolazione. New York è stata particolarmente colpita. L'AEI Housing Center ha rilevato che tra maggio e agosto 2020 gli acquirenti hanno optato per abitazioni in aree con una densità media del 19\% inferiore rispetto allo stesso periodo dell'anno precedente. La domanda è aumentata del $74 \%$ nel quintile meno denso dei codici di avviamento postale. La maggior parte del calo è stato a scapito del quintile più denso (-3,9 punti percentuali) (American Enterprise Institute, 2020[49]). Secondo altre fonti, da 14 a 23 milioni di americani potrebbero avere in programma di spostarsi a causa del lavoro a distanza. Insieme a coloro che si spostano indipendentemente dal lavoro a distanza, i tassi di migrazione a breve termine possono essere da tre a quattro volte superiori a quelli normali. Le grandi città registrerebbero la maggiore emigrazione: il $20,6 \%$ di coloro che pianificano di trasferirsi hanno attualmente sede in una grande città. Più della metà $(54,7 \%)$ si sposta a più di due ore di distanza dalla propria posizione attuale, che per la gran parte di essi è oltre la distanza coperta giornalmente o addirittura settimanalmente per gli spostamenti da e verso il posto di lavoro (Upwork, 2020[50]).

Tuttavia, un declino delle città non è né plausibile né auspicabile. L'analisi mostra che la densità urbana, così come la dimensione della città, tende ad aumentare la produttività (OECD, 2015 [51]; Harding, 2020[52]). 
I grandi centri urbani consentono di realizzare economie di scala che rendono più efficiente la fornitura di alcuni servizi - ad esempio i porti merci e passeggeri, i servizi specializzati e le attività di intrattenimento. Poiché non tutti i servizi possono essere prodotti o consumati a distanza, un declino dei grandi centri urbani comporterebbe una perdita di economie di scala e di servizi che potrebbero ridurre la produttività, l'occupazione e le scelte dei consumatori in alcuni settori (servizi alla persona, servizi alimentari, vendita al dettaglio, ecc.).

Una ricerca che intende modellare gli effetti a lungo termine del telelavoro di massa sulle strutture urbane mostra i potenziali impatti sulle decisioni di localizzazione delle aziende e delle famiglie e sui prezzi degli immobili. In particolare, un aumento del telelavoro potrebbe contribuire ad un calo dei prezzi delle abitazioni, in quanto i telelavoratori lascerebbero i quartieri centrali per acquistare case più grandi in periferia. I tempi per gli spostamenti potrebbero diminuire a causa della riduzione della congestione del traffico e le disuguaglianze salariali tra i quartieri si ridurrebbero, poiché i lavoratori ad alto reddito potrebbero vivere più lontano dai loro luoghi di lavoro, favorendo una maggiore compresenza tra diversi livelli di reddito (Parkhomenko A. et al., 2020[53]).

Allo stesso modo, i recenti annunci delle grandi aziende tecnologiche di voler continuare con il telelavoro di massa anche dopo la pandemia potrebbero aprire la strada non solo al telelavoro localizzato, ma anche a un più ampio decentramento dell'occupazione americana a più alto valore aggiunto lontano dalle più grandi aree metropolitane e verso quelle più centrali e a basso costo. Tale tendenza sarebbe tanto più interessante se si considera che né le dinamiche di mercato né gli sforzi di sviluppo economico dal basso verso l'alto sono riusciti a colmare questo divario nel corso degli anni (Muro M., 2020[54]).

In Italia, il tema delle migrazioni per telelavoro verso le regioni in ritardo di sviluppo è stato oggetto di particolare attenzione. Alcuni osservatori sottolineano che le regioni economicamente svantaggiate del Sud potrebbero offrire ai lavoratori a distanza un costo della vita più basso rispetto al Nord, con una presenza del 4G uguale o leggermente migliore (D'Alessandro J., 2020[55]). Tuttavia, i dati sulla quota di famiglie con la banda larga sembrano contraddire queste ipotesi (Bonini, 2020[56]).

La letteratura accademica sulle tendenze migratorie verso le località periferiche evidenzia che lo spopolamento non è un fenomeno irreversibile. A partire dagli anni Novanta ad esempio, un numero sempre maggiore di comunità alpine in Europa, che per molti decenni hanno sofferto l'emigrazione, sono diventate meta di immigrazione. Questa nuova tendenza demografica è particolarmente pronunciata nelle Alpi occidentali francesi e italiane e nei dintorni delle Alpi centrali (Löffler et al., 2016[57]). II crescente scollamento tra la posizione fisica dei datori di lavoro e quella dei dipendenti, causato da una transizione di massa verso il telelavoro, potrebbe aggiungere nuovo slancio a questa tendenza.

Il telelavoro permanente su larga scala che modifica la distribuzione territoriale dei diversi tipi di occupati può anche alterare le tendenze elettorali. Ad esempio, il declino economico e industriale è correlato al voto anti-UE. Le aree con tassi di occupazione più bassi o una forza lavoro meno istruita hanno maggiori probabilità di votare contro l'UE, rafforzando il nesso tra demografia, geografia e comportamento elettorale (Dijkstra L. et al., 2018[58]). In altre parole, il telelavoro di massa potrebbe alterare la cosiddetta "geografia del malcontento".

Tuttavia, le proiezioni post-COVID dovrebbero tener conto della misura in cui le quote delle occupazioni che possono essere svolte a distanza possono differire in modo significativo all'interno dei Paesi. Le regioni dove è localizzata la capitale presentano, nella maggior parte dei casi, un più elevato potenziale nel lavoro a distanza, pari a 9 punti percentuali in più rispetto alla media nazionale. Nel complesso, le differenze regionali nel potenziale di telelavoro sono nette. In media, si rileva una differenza di 15 punti percentuali tra la regione con il livello più alto e quella con il livello più basso in un dato Paese. II potenziale di lavoro a distanza è più alto anche nelle aree più densamente popolate. Le città hanno una quota di posti di lavoro che si possono svolgere a distanza superiore di 13 punti percentuali rispetto alle aree rurali. Infine, i tassi di lavoro a distanza nelle regioni riflettono la composizione delle competenze della forza lavoro locale, in quanto gli occupati con qualifiche più elevate sono più adattabili al telelavoro. Anche se altri fattori, come 
la composizione della base industriale, possono giocare un ruolo importante, le evidenze mostrano che la quota di lavoratori con istruzione terziaria sembra essere fortemente correlata con la quota di occupazioni che si possono svolgere a distanza (OECD, 2020 $\left.\mathrm{e}_{[59]}\right)$. Uno studio dell'Office for National Statistics del Regno Unito conferma che le opportunità di lavoro da casa variano in modo significativo tra settori e regioni, a seconda del tipo di attività, della diffusione delle competenze digitali e della disponibilità di infrastrutture informatiche (Office for National Statistics, 2020[60]]).

\section{Come il telelavoro di massa potrebbe influire sulle imprese di diverse dimensioni}

Analogamente ai territori, le imprese non sono attrezzate in uguale misura per gestire il telelavoro, e questo vale in particolare per le PMI. I sondaggi condotti durante la pandemia di COVID-19 in tutto il mondo forniscono una grande quantità di dati sull'adozione del telelavoro e di altre pratiche digitali (ad esempio e-commerce, sicurezza informatica, ecc.) da parte degli intervistati. Tali fonti evidenziano il rischio di perpetuare le disparità tra imprese in base alle loro dimensioni. Le PMI sostengono costi più che proporzionatamente elevati per l'adozione del telelavoro e gli investimenti complementari (ad esempio la sicurezza informatica) rispetto alle imprese più grandi. Gli svantaggi competitivi potrebbero essere avvertiti maggiormente dalle imprese più piccole situate in zone con una minore diffusione delle reti internet ad alta velocità. Inoltre, il brusco passaggio al telelavoro nel settore pubblico potrebbe portare a interruzioni e ritardi nella fornitura di servizi pubblici e nelle procedure amministrative (autorizzazioni, licenze, incentivi, appalti), con un impatto assai maggiore per le PMI.

- Stati Uniti: un'indagine pubblicata dalla Camera di Commercio degli Stati Uniti il 5 maggio 2020 ha evidenziato un'accelerazione dei processi di digitalizzazione. La quota di piccole imprese che hanno iniziato a trasferire alcuni, o tutti, i loro dipendenti verso una modalità di lavoro a distanza è aumentata dal $12 \%$ al $20 \%$, e le piccole imprese che hanno iniziato a spostare la loro attività commerciale verso i canali digitali sono passate dal 10\% al 17\% (US Chamber of Commerce, 2020[61]).

- Giappone: secondo i rapporti dell'11 maggio 2020, un'indagine gestita dalla Camera di Commercio di Tokyo e Osaka ha evidenziato un divario nella prevalenza del telelavoro per dimensione aziendale (48\% per le grandi imprese contro il $10-20 \%$ delle PMI), dovuto alla mancanza di infrastrutture e di competenze digitali tra le imprese più piccole (The Japan Times, 2020[62]).

- Germania: un'indagine condotta all'inizio di maggio 2020 ha evidenziato che mentre all'inizio della crisi I' $88 \%$ delle PMI tedesche operava attraverso modalità di lavoro da svolgersi esclusivamente di persona, l' $81 \%$ prevede che la pandemia renderà le proprie aziende più flessibili e un terzo delle PMI ritiene che la digitalizzazione sia cresciuta di importanza a causa della pandemia (McKinsey, $\left.2020 b_{[63]}\right)$.

- Europa: un ulteriore studio sull'Europa ha suggerito che solo il $56 \%$ di tutte le aziende con 50 o meno dipendenti ha fornito ai propri dipendenti accesso remoto alla posta elettronica, alle applicazioni e ai documenti, rispetto al 93\% di tutte le aziende con più di 250 dipendenti (McKinsey, $\left.2020 c_{[64]}\right)$.

- Francia: un sondaggio dell'aprile 2020 condotto da un'agenzia elettorale francese ha evidenziato che le dimensioni dell'azienda implicano enormi differenze nell'adozione del telelavoro nel Paese. Durante la fase iniziale della pandemia, i lavoratori da casa rappresentavano il $17 \%$ dei lavoratori nelle imprese con meno di dieci dipendenti, contro il $38 \%$ nelle imprese con più di cinquemila (ODOXA, 2020b[37]).

Alla luce dell'espansione del telelavoro, sarà necessario compiere degli sforzi per mantenere la competitività delle PMI. A causa delle lacune nei finanziamenti, nelle capacità di gestione e nella ridotta capacità di beneficiare delle economie di scala, le PMI potrebbero essere maggiormente soggette alle sfide derivanti dal telelavoro e meno in grado di trarre vantaggio dai suoi punti di forza. Come si è visto 
nelle sezioni precedenti, questi comprendono minori costi per gli immobili ad uso ufficio e una riduzione dell'assenteismo. Inoltre, ci sono indicazioni di un aumento della produttività e della soddisfazione dei dipendenti quando i livelli di telelavoro sono bassi o intermedi. Al contrario, le sfide includono costi più elevati per la formazione e le attrezzature digitali e la ridotta capacità dei manager di supervisionare $\mathrm{i}$ dipendenti, mentre livelli "eccessivi" di telelavoro potrebbero influire sul benessere dei lavoratori, rallentare la produttività e ridurre lo spirito di squadra. Sono tuttavia necessarie ulteriori evidenze per determinare l'influenza del telelavoro sulla produttività e i diversi impatti rispetto al tipo di attività o dimensione dell'impresa.

\section{La necessità di un approccio di policy lungimirante e basato sulle evidenze}

II rischio che crescano gli squilibri è un filo conduttore che riguarda tutte le categorie analizzate finora, ovvero i gruppi sociali (datori di lavoro e dipendenti), i territori (mercati del lavoro locali) e le imprese. Si profilano due tendenze in qualche modo opposte. Da un lato, le disparità preesistenti tra i gruppi di persone (lavoratori altamente e scarsamente qualificati) e le imprese (grandi e piccole e medie imprese) potrebbero aumentare in futuro, sulla base dei differenziali nella disponibilità ad adottare il telelavoro. Dall'altro lato, i luoghi meno performanti potrebbero trovare nuove opportunità per recuperare il ritardo - come nel caso delle aree rurali, che potrebbero attrarre quote crescenti di telelavoratori e trattenere i residenti, che potrebbero accedere a posti di lavoro a distanza senza perdere il loro legame con la comunità locale.

La presenza di evidenze contrastanti su questioni quali la produttività e il benessere dei dipendenti, insieme alla gamma delle questioni in gioco, richiede l'adozione di un approccio di policy graduale. Tale approccio dovrebbe essere informato dai dati e sostenuto da un dialogo continuo con le parti sociali e gli altri soggetti interessati. A livello di gestione aziendale, l'Organizzazione Internazionale del Lavoro promuove un approccio "test-and-learn" basato sull'identificazione di obiettivi, compiti e traguardi, nonché sul monitoraggio e la discussione dei progressi compiuti - un approccio che potrebbe essere applicabile anche alla definizione delle politiche (ILO, 2020b[65]).

La Tabella 1 delinea, senza pretendere di essere esaustiva, alcune delle principali questioni di policy alla base di una potenziale diffusione di massa del telelavoro. 
Tabella 1. Principali questioni politiche e opportunità e minacce più frequentemente associate al telelavoro (dipendenti, datori di lavoro, aziende, aree urbane, aree rurali, società)

\begin{tabular}{|c|c|c|c|}
\hline & Questione politica & Opportunità & Minacce \\
\hline \multirow[t]{3}{*}{ Telelavoratori } & $\begin{array}{l}\text { II telelavoro migliora l'equilibrio tra } \\
\text { lavoro e vita privata? }\end{array}$ & $\begin{array}{l}\text { Migliore adattabilità del ciclo di } \\
\text { lavoro alle esigenze personali } \\
\text { Soluzione a misura di famiglia, ad } \\
\text { esempio più flessibilità per i genitori } \\
\text { Tempo risparmiato per gli } \\
\text { spostamenti da e verso il posto di } \\
\text { lavoro }\end{array}$ & $\begin{array}{l}\text { Confini sfuocati tra vita e lavoro } \\
\text { Aumento autoindotto del carico di } \\
\text { lavoro } \\
\text { Maggiori oneri per l'assistenza } \\
\text { all'infanzia per le donne, } \\
\text { perpetuando le disparità preesistenti } \\
\text { Difficoltà nel misurare e riportare gli } \\
\text { straordinari }\end{array}$ \\
\hline & $\begin{array}{l}\text { II telelavoro comporta risparmi per i } \\
\text { dipendenti? }\end{array}$ & $\begin{array}{l}\text { Minori spese diurne per il cibo } \\
\text { Minori spese per l'alloggio (maggiore } \\
\text { flessibilità nella scelta del quartiere } \\
\text { per l'abitazione) }\end{array}$ & $\begin{array}{l}\text { Maggiori costi domestici per } \\
\text { l'elettricità, le strutture informatiche, } \\
\text { lo spazio di lavoro, ecc. }\end{array}$ \\
\hline & $\begin{array}{l}\text { Il telelavoro influisce sulle } \\
\text { prospettive di carriera? }\end{array}$ & $\begin{array}{l}\text { Maggiore fiducia tra datore di lavoro } \\
\text { e dipendente }\end{array}$ & $\begin{array}{l}\text { Minore visibilità, promozioni, aumenti } \\
\text { di stipendio } \\
\text { Minori opportunità di networking } \\
\text { all'interno dell'azienda } \\
\text { Riduzione dell'accesso alla } \\
\text { formazione e ai flussi di conoscenza }\end{array}$ \\
\hline \multirow[t]{3}{*}{ Manager } & II telelavoro migliora le prestazioni? & Riduzione delle assenze & Minore supervisione dei dipendenti \\
\hline & $\begin{array}{l}\text { Il telelavoro riduce i costi dei datori di } \\
\text { lavoro? }\end{array}$ & $\begin{array}{l}\text { Spese minori per i locali di lavoro, } \\
\text { parziale dismissione delle strutture }\end{array}$ & $\begin{array}{l}\text { Costi più elevati per la formazione } \\
\text { digitale }\end{array}$ \\
\hline & $\begin{array}{l}\text { Il telelavoro aumenta lo spirito di } \\
\text { squadra? }\end{array}$ & $\begin{array}{l}\text { Maggiore soddisfazione tra i } \\
\text { dipendenti (bassa intensità di } \\
\text { telelavoro) } \\
\text { Maggiore fidelizzazione dei } \\
\text { dipendenti }\end{array}$ & Diminuzione dello spirito di squadra \\
\hline Imprese & $\begin{array}{l}\text { Le imprese sono tutte attrezzate per } \\
\text { passare al telelavoro su larga scala? }\end{array}$ & $\begin{array}{l}\text { Riduzione del costo marginale per le } \\
\text { infrastrutture digitali } \\
\text { Spill-over digitali, ovvero ricadute in } \\
\text { termini di miglioramento delle } \\
\text { competenze e delle infrastrutture } \\
\text { digitali }\end{array}$ & $\begin{array}{l}\text { Costi sproporzionatamente più } \\
\text { elevati per le PMI nell'adozione del } \\
\text { digitale } \\
\text { Svantaggio competitivo per le } \\
\text { imprese situate in aree con scarse } \\
\text { infrastrutture IT } \\
\text { Prevalenza di compiti che non si } \\
\text { prestano al telelavoro in alcuni } \\
\text { settori industriali }\end{array}$ \\
\hline Aree urbane & $\begin{array}{l}\text { In che modo il telelavoro di massa } \\
\text { influisce sulle aree densamente } \\
\text { popolate? }\end{array}$ & $\begin{array}{l}\text { Riduzione del traffico, meno } \\
\text { pressione sulle infrastrutture di } \\
\text { trasporto locali } \\
\text { Prezzi d'affitto più bassi se meno } \\
\text { persone cercano un alloggio nella } \\
\text { zona } \\
\text { Maggiore coesione con le aree rurali }\end{array}$ & $\begin{array}{l}\text { Calo della domanda di servizi } \\
\text { pubblici e privati } \\
\text { Svalutazione di investimenti } \\
\text { immobiliari in aree urbane } \\
\text { Fuoriuscita di capitale umano }\end{array}$ \\
\hline Zone rurali & $\begin{array}{l}\text { In che modo il telelavoro di massa } \\
\text { interessa le aree meno densamente } \\
\text { popolate? }\end{array}$ & $\begin{array}{l}\text { Nuove opportunità di lavoro, } \\
\text { mantenimento dei posti di lavoro } \\
\text { Afflusso di capitale umano, } \\
\text { ripopolamento } \\
\text { Maggiore base imponibile per } \\
\text { finanziare i servizi pubblici } \\
\text { Incentivo per accelerare gli } \\
\text { investimenti in infrastrutture IT } \\
\text { Maggiore coesione con le aree } \\
\text { urbane }\end{array}$ & $\begin{array}{l}\text { Inizialmente, minore disponibilità di } \\
\text { servizi pubblici e privati locali } \\
\text { Divario digitale preesistente con le } \\
\text { aree urbane } \\
\text { Rischio di un aumento eccessivo dei } \\
\text { prezzi d'affitto/spostamento della } \\
\text { popolazione locale }\end{array}$ \\
\hline
\end{tabular}




\begin{tabular}{l|l|l|l}
\hline & \multicolumn{1}{|c|}{ Questione politica } & \multicolumn{1}{c}{ Opportunità } & \multicolumn{1}{c}{ Minacce } \\
\hline Società & $\begin{array}{l}\text { Che impatto ha il telelavoro di massa } \\
\text { sulla società nel suo complesso? }\end{array}$ & $\begin{array}{l}\text { Meno emissioni di gas serra grazie } \\
\text { alla riduzione degli spostamenti da e } \\
\text { verso il posto di lavoro e dei viaggi di } \\
\text { lavoro } \\
\text { Miglioramento del benessere }\end{array}$ & $\begin{array}{l}\text { Aumento dell'impatto climatico dei } \\
\text { data centre } \\
\text { Costi potenzialmente maggiori per } \\
\text { l'assistenza sanitaria: sedentarietà, } \\
\text { ansia, isolamento sociale }\end{array}$ \\
& $\begin{array}{l}\text { Migliore sostenibilità del welfare (ad } \\
\text { Disparità di accesso alle opportunità } \\
\text { (lavoratori altamente o scarsamente } \\
\end{array}$ & $\begin{array}{l}\text { l'assistenza all'infanzia) } \\
\text { Miglioramento della coesione } \\
\text { qualificati, settori online o offline) }\end{array}$ & $\begin{array}{l}\text { Rischio di un aumento della violenza } \\
\text { domestica }\end{array}$ \\
\hline
\end{tabular}

Fonte: Elaborazione dell'autore.

Nel contesto della pandemia, i governi regionali e locali stanno adottando importanti azioni a breve termine per adattarsi all'economia del distanziamento sociale. Oltre a contribuire alla risposta sanitaria, comprese le attività di sensibilizzazione, la fornitura di assistenza sanitaria e il sostegno ai gruppi vulnerabili, essi stanno svolgendo un ruolo importante nel fornire risposte complementari ai piani nazionali per i sussidi di disoccupazione e il sostegno al reddito, in particolare per preservare le PMI e i posti di lavoro ad esse associati. Una necessità primaria in questo senso è equipaggiare le aziende per consentirne l'accesso al telelavoro, ed in particolare le micro e piccole imprese.

La sezione seguente fornisce una panoramica delle varie pratiche di policy per promuovere il telelavoro messe in atto a diversi livelli di governo, sia prima che durante la pandemia. La consapevolezza delle diverse opzioni disponibili e delle loro implicazioni può guidare i decisori politici nella progettazione di una combinazione di politiche efficace e adeguata alle caratteristiche e alle esigenze locali. 


\section{Politiche per il telelavoro}

\section{Un insieme differenziato di politiche su più livelli per promuovere il telelavoro}

Durante la pandemia di COVID-19 tutti i livelli di governo sono stati chiamati ad attuare misure a sostegno del telelavoro. I governi nazionali, regionali e locali hanno introdotto una serie di politiche per superare le diverse sfide e fornire risposte specifiche.

La Tabella 2 fornisce una panoramica delle diverse tipologie di risposte di policy riguardanti il telelavoro descritte in questa sezione e la loro distribuzione tra Paesi, Regioni e Città. Esse sono presentate in ordine crescente rispetto al coinvolgimento delle imprese ai fini della loro attuazione, dalla semplice promozione governativa del telelavoro (tipologie 1 e 2) e interventi normativi per consentire il ricorso a questa modalità di lavoro (da 3 a 6 ), a misure non finanziarie (da 7 a 9) e di sostegno finanziario per promuovere l'adozione del telelavoro da parte delle PMI (10 e 11), fino a programmi per l'attrazione dei lavoratori a distanza (da 12 a 15).

Da un lato, i governi nazionali hanno svolto un ruolo importante nel fornire l'infrastruttura normativa (sia soft che vincolante, sia aggiornata che nuova) e digitale (ad esempio le piattaforme per i servizi) per consentire l'utilizzo telelavoro. Dall'altro lato, le autorità locali sono state particolarmente attive nell'attuazione di misure non finanziarie (campagne informative e formazione) e di sostegno finanziario (tipicamente sovvenzioni per il rimborso dei costi sostenuti per gli investimenti digitali) volte a promuovere l'adozione del telelavoro tra le PMI.

II modello di distribuzione delle tipologie di policy tra le regioni sembra essere correlato al grado di maturità digitale a livello locale. Alcune regioni che ospitano una forte comunità digitale hanno persino iniziato a lavorare ai primi esempi di piani a lungo termine per il telelavoro, mentre altre hanno dovuto dare priorità alle campagne informative e alla formazione digitale per rendere il telelavoro accessibile a tutti.

Saranno analizzati anche diversi casi di politiche riguardanti il telelavoro adottate prima della pandemia, data la loro maggiore rilevanza e il bagaglio di conoscenze ed esperienze che possono fornire. Negli ultimi anni i Paesi e le regioni di tutto il mondo hanno lanciato diversi programmi per favorire l'attrazione di lavoratori o imprenditori digitali e a distanza di talento, attraverso l'erogazione di borse di studio, strutture di co-working, visti start-up e altri strumenti (tipologie n. 12-15). Altre autorità locali hanno istituito misure e incentivi per le imprese home-based, che potrebbero acquisire nuovo slancio nel contesto attuale (n. 9). A parte queste poche eccezioni, tutti gli altri tipi di politiche elencate qui di seguito sono state introdotte durante la pandemia. 
Tabella 2. Tipologie di politiche per il telelavoro, per Paese, Regione e Città

\begin{tabular}{|c|c|c|c|}
\hline$\#$ & Tipologia di politica & Esempi di Paesi & Esempi di Regioni e Città \\
\hline 1 & $\begin{array}{l}\text { Raccomandazioni per adottare il telelavoro } \\
\text { durante la pandemia }\end{array}$ & $\begin{array}{l}\text { Australia, Austria, Canada, Nuova } \\
\text { Zelanda, Polonia, Portogallo, Slovacchia, } \\
\text { Spagna, Svizzera }\end{array}$ & \\
\hline 2 & $\begin{array}{l}\text { Piani a lungo termine per mantenere il } \\
\text { telelavoro come opzione preferenziale su } \\
\text { base permanente }\end{array}$ & & $\begin{array}{l}\text { Milano (Italia), Contea di Santa Clara - } \\
\text { California (Stati Uniti) }\end{array}$ \\
\hline 3 & $\begin{array}{l}\text { Regolamentazioni soft sul telelavoro, ad } \\
\text { es. linee guida }\end{array}$ & $\begin{array}{l}\text { Australia, Belgio, Canada, Francia, Grecia, } \\
\text { Stati Uniti }\end{array}$ & \\
\hline 4 & $\begin{array}{l}\text { Modifiche alla normativa preesistente in } \\
\text { materia di telelavoro }\end{array}$ & $\begin{array}{l}\text { Australia, Colombia, Corea del Sud, } \\
\text { Francia, Italia, Slovenia, Ungheria }\end{array}$ & \\
\hline 5 & Nuove norme sul telelavoro & Cile & \\
\hline 6 & $\begin{array}{l}\text { Accordi fiscali di reciprocità per i lavoratori } \\
\text { frontalieri tenuti a telelavorare durante il } \\
\text { lockdown }\end{array}$ & $\begin{array}{l}\text { Belgio, Francia, Germania, Lussemburgo, } \\
\text { Paesi Bassi, Svizzera }\end{array}$ & $\begin{array}{l}\text { Indiana, Maryland, Minnesota, Mississippi, } \\
\text { New Jersey, North Dakota, Ohio (Stati } \\
\text { Uniti) }\end{array}$ \\
\hline 7 & $\begin{array}{l}\text { Piattaforme online per il telelavoro e i } \\
\text { servizi digitali }\end{array}$ & $\begin{array}{l}\text { Austria, Canada, Irlanda, Italia, Lettonia, } \\
\text { Malesia, Messico, Portogallo }\end{array}$ & Québec (Canada) \\
\hline 8 & $\begin{array}{l}\text { Campagne informative e formazione sul } \\
\text { telelavoro per le PMI }\end{array}$ & & $\begin{array}{l}\text { Abruzzo (Italia), Braga (Portogallo), } \\
\text { Barcellona, Paesi Baschi (Spagna) }\end{array}$ \\
\hline 9 & $\begin{array}{l}\text { Formazione professionale a distanza per le } \\
\text { PMI }\end{array}$ & Turchia & $\begin{array}{l}\text { Île-de-France, Normandia, Occitania } \\
\text { (Francia), Friuli-Venezia Giulia, Liguria } \\
\text { (Italia) }\end{array}$ \\
\hline 10 & $\begin{array}{l}\text { Politiche a sostegno delle imprese home- } \\
\text { based }\end{array}$ & $\begin{array}{l}\text { Australia, Paesi Bassi, Regno Unito, Stati } \\
\text { Uniti, }\end{array}$ & $\begin{array}{l}\text { Victoria (Australia), Vienna (Austria), } \\
\text { Alberta, Ottawa (Canada), Barcellona } \\
\text { (Spagna), Bristol, Scozia (Regno Unito) }\end{array}$ \\
\hline 11 & $\begin{array}{l}\text { Sostegno finanziario alle PMI per } \\
\text { l'adozione del telelavoro }\end{array}$ & Cina, Giappone, Spagna & $\begin{array}{l}\text { Stiria, Tirolo (Austria), Basilicata, Friuli- } \\
\text { Venezia Giulia, Liguria, Lombardia, Molise, } \\
\text { Piemonte, Puglia (Italia), Paesi Baschi } \\
\text { (Spagna), Tokyo (Giappone) }\end{array}$ \\
\hline 12 & $\begin{array}{l}\text { Sovvenzioni per attirare i lavoratori a } \\
\text { distanza }\end{array}$ & & Vermont, Tulsa - Oklahoma (Stati Uniti) \\
\hline 13 & $\begin{array}{l}\text { Strutture di co-working per attirare i } \\
\text { lavoratori da remoto }\end{array}$ & & $\begin{array}{l}\text { Wittemberge e Alta Lusazia - Sassonia, } \\
\text { Eberswalde - Brandeburgo, Schleswig- } \\
\text { Holstein (Germania), Milano (Italia) }\end{array}$ \\
\hline 14 & Residenza digitale & Estonia & \\
\hline 15 & $\begin{array}{l}\text { Start-up visa per attirare gli imprenditori del } \\
\text { digitale }\end{array}$ & $\begin{array}{l}\text { Cile e molti altri Paesi }(\approx 50 \text { in tutto il } \\
\text { mondo) }\end{array}$ & \\
\hline
\end{tabular}

Fonte: Elaborazione dell'autore.

Ciascuna delle seguenti sottosezioni analizza una specifica categoria di misure, identificata in base al tipo di intervento e alla logica perseguita. Nell'Allegato A. Una rassegna delle politiche nazionali, regionali e locali per il telelavoro è poi disponibile un elenco completo delle misure, suddivise per Paese, Regione e Città di attuazione, con una breve descrizione e i riferimenti associati a ciascuna voce.

\section{Dalle raccomandazioni a breve termine ai piani a lungo termine per l'adozione del telelavoro}

Con l'esplosione della pandemia i governi nazionali hanno intrapreso ampie azioni per raccomandare l'adozione di accordi per il telelavoro tra i datori di lavoro privati, spesso dando l'esempio con il personale della pubblica amministrazione. Particolarmente degno di nota in questo senso è il caso del Canada, il cui governo federale ha messo in atto una serie di iniziative per migliorare la gestione del telelavoro per il 
personale dei servizi essenziali della pubblica amministrazione e per garantire che i dipendenti abbiano gli strumenti necessari per lavorare in sicurezza da casa.

Il telelavoro è stato promosso da diversi Paesi in tutto il mondo, tra cui Australia, Austria, Canada, Francia, Grecia, Nuova Zelanda, Polonia, Portogallo, Slovacchia, Spagna e Svizzera. Nella maggior parte di questi Paesi, ciò è andato di pari passo con l'adozione di misure di contenimento per ridurre i rischi di contagio, e solo le aziende che soddisfacevano i requisiti di classificazione per un'attività essenziale sono state autorizzate a rimanere aperte. La flessibilità è il principio fondamentale soggiacente a questi sforzi. II governo greco, ad esempio, ha promosso accordi sul telelavoro consentendo ai datori di lavoro privati di sviluppare politiche per il telelavoro nell'ambito del quadro fornito dal Ministero del Lavoro. I datori di lavoro dovranno solo dare notizia di tali accordi attraverso il sistema informatico del Ministero del Lavoro per ciascuno dei lavoratori coinvolti.

Alcuni governi locali hanno successivamente emanato piani a più lungo termine, incoraggiando il telelavoro anche dopo la fine della pandemia. La Città di Milano (Italia) e la Contea di Santa Clara, in California (Stati Uniti) ne sono due esempi.

Nell'aprile 2020 il Comune di Milano ha avviato una consultazione pubblica su una bozza di piano di rilancio post-COVID dal titolo "Milano 2020. Strategia di adattamento". II documento sottolinea la necessità di mantenere il telelavoro come scenario privilegiato nel breve e medio termine. Nel maggio 2020 l'amministrazione comunale ha concordato con i maggiori datori di lavoro privati locali che l' $80 \%$ della loro forza lavoro continuerà a lavorare da casa nei prossimi mesi.

Nel maggio 2020 il Consiglio dell'Autorità di Vigilanza della Contea di Santa Clara ha approvato una raccomandazione per incoraggiare ulteriormente il telelavoro dopo la fine della crisi dovuta al COVID-19. Il disegno di legge riconosce i benefici avvenuti nella Bay Area, dovuti al miglioramento della qualità dell'aria e alla riduzione del traffico da quando sono in vigore le politiche di contenimento e molti dipendenti hanno iniziato a lavorare da casa. L'obiettivo a lungo termine è quello di mantenere elevati livelli di produttività, riducendo al contempo l'impatto sul clima. Coinvolgere i datori di lavoro privati nella riduzione delle emissioni di gas serra è un altro importante obiettivo. È stata proposta la realizzazione di un progetto pilota, chiamato "Clear Air Telecommute Challenge" volto a sensibilizzare i grandi datori di lavoro ad aumentare la quota di dipendenti che telelavorano. II Silicon Valley Leadership Group, un'organizzazione per le politiche pubbliche che rappresenta più di 350 aziende, ha espresso sostegno all'idea e ha raccomandato la partecipazione del settore privato alla formulazione del piano.

\section{Messa a punto di un quadro normativo per il telelavoro}

II COVID-19 ha fatto sì che diversi Paesi adottassero nuove norme sul telelavoro o modificassero quelle esistenti, creando una maggiore consapevolezza riguardo alle stesse. I governi nazionali hanno assunto un ruolo importante in questo ambito, mentre le regioni e i governi locali hanno per lo più integrato tali azioni attraverso l'attuazione delle normative nazionali a livello locale.

In Cile sono state annunciate nuove normative incentrate sul telelavoro e sui diritti dei telelavoratori. La legge consente ai lavoratori e alle imprese di trovare accordi sul telelavoro, chiarisce gli obblighi dei datori di lavoro in termini di prevenzione dei rischi e di protezione dei lavoratori e stabilisce il diritto di disconnettersi per i dipendenti.

Diversi Paesi hanno introdotto norme temporanee o ampliato il campo di applicazione di quelle esistenti per facilitare il telelavoro durante la pandemia di COVID-19. Tra questi vi sono Australia, Colombia, Corea del Sud, Francia, Italia e Ungheria. In Australia, la diffusione del telelavoro sta portando i decisori politici a considerare la possibilità di modificare le leggi sul lavoro che regolano gli aspetti delle relazioni tra datore di lavoro e dipendente e i comportamenti appropriati sul posto di lavoro per affrontare in modo più efficace le situazioni in cui la forza lavoro non è presente sul luogo di lavoro. Ad esempio, attualmente la notifica 
della cessazione del rapporto di lavoro deve avvenire faccia a faccia piuttosto che in forma scritta o attraverso altre comunicazioni elettroniche. La Colombia ha pubblicato una nota con disposizioni specifiche su lavoro da casa, telelavoro, orario di lavoro flessibile e ferie anticipate o collettive. L'Ungheria ha temporaneamente modificato la legislazione sul lavoro per estendere il telelavoro, il lavoro da casa e l'orario di lavoro flessibile. L'Italia ha introdotto disposizioni che consentono modalità semplificate per il telelavoro per tutti i settori ad esso accessibili; procedure semplificate per le richieste e la notifica del telelavoro; procedure di gara semplificate per il settore pubblico. La Corea del Sud ha temporaneamente semplificato le procedure di richiesta di un contributo per i datori di lavoro (fino a 2 milioni di won, cioè circa 1500 euro all'anno per dipendente) per l'introduzione di modalità di lavoro flessibili come il lavoro da casa, il lavoro a distanza o l'orario di lavoro selettivo.

In molti Paesi queste norme sono state accompagnate da attività di sensibilizzazione e dallo sviluppo di linee guida pratiche. È il caso di Australia, Belgio, Francia, Grecia e Stati Uniti, che hanno pubblicato delle linee guida per i datori di lavoro e i lavoratori per capire come migliorare l'attuazione del telelavoro, dato che la legge consente già questa modalità. Degna di nota, tra le linee guida pubblicate sul telelavoro, quella dell'Autorità Ellenica per la Protezione dei Dati che ha pubblicato, nell'aprile 2020, delle linee guida sul telelavoro per garantire la sicurezza dei dati e il rispetto del Regolamento Generale sulla Protezione dei Dati dell'Unione Europea. II tribunale australiano indipendente per le relazioni industriali, la Fair Work Commission (FWC), ha temporaneamente modificato una serie di tutele che fanno parte della rete di sicurezza del lavoro australiana in risposta alla pandemia di COVID-19. Ciò include meccanismi che consentono forme di flessibilità temporanee per facilitare il lavoro da casa in alcuni settori. Prendendo atto della maggiore diffusione degli accordi di lavoro da casa durante questo periodo, la FWC ha pubblicato una bozza di schema di flessibilità dei modelli di tutela come punto di partenza per la discussione tra le parti interessate al fine di garantire che tali tutele rispondano alle esigenze di flessibilità per gli accordi di lavoro.

L'estensione della durata del telelavoro durante la pandemia ha anche sollevato questioni sullo status dei lavoratori frontalieri, portando i governi ad aggiornare i regolamenti e gli accordi bilaterali in materia. Nel contesto UE, questo termine si riferisce ai lavoratori che sono impiegati nella zona di frontiera di uno Stato membro dell'UE e ritornano ogni giorno o almeno una volta alla settimana nella zona di frontiera di un Paese confinante in cui risiedono e di cui sono cittadini. Alcuni Paesi europei, tra cui Austria, Belgio, Francia, Germania, Lussemburgo, Paesi Bassi e Svizzera, hanno concluso accordi bilaterali per stabilire regimi fiscali specifici per i lavoratori frontalieri. Ad esempio, la Germania ha concluso accordi reciproci con Austria, Lussemburgo e Paesi Bassi, che dovrebbero contribuire a far sì che i lavoratori frontalieri che attualmente lavorano da casa a causa del COVID-19 possano ancora beneficiare delle agevolazioni fiscali concesse dalla Germania. In Lussemburgo, la regola generale stabilisce che i lavoratori siano tassati nel Paese in cui lavorano, mentre i lavoratori frontalieri francesi possono lavorare fino a 29 giorni in Francia per il loro datore di lavoro lussemburghese senza che la retribuzione diventi imponibile in Francia. La Francia e il Lussemburgo hanno convenuto che l'aumento del numero di giorni che i lavoratori frontalieri hanno lavorato da casa dovuto al COVID-19 non sarà preso in considerazione per il calcolo del limite di 29 giorni. Diversi stati degli Stati Uniti (tra cui Indiana, Maryland, Minnesota, Mississippi, New Jersey, North Dakota e Ohio) hanno pubblicato una guida sull'impatto che i disagi dovuti al COVID-19 avranno sugli obblighi di ritenuta per i datori di lavoro che operano in diversi stati.

\section{Piattaforme pubblico-private per il telelavoro e i servizi digitali}

Durante la crisi dovuta al COVID-19 diversi governi nazionali hanno creato piattaforme online per favorire l'accesso di aziende e cittadini agli strumenti digitali. Tali iniziative sono particolarmente rilevanti per le $\mathrm{PMI}$, che spesso sono in ritardo rispetto alle imprese di maggiori dimensioni in termini di accesso alle tecnologie digitali. 
Due esempi di rilievo provengono da Austria e Italia. II Ministero Federale Austriaco per lo Sviluppo Digitale ed Economico ha incoraggiato la creazione del "Digital Team Austria", un gruppo di aziende IT che si impegnano ad offrire gratuitamente servizi digitali alle PMI specificamente progettati per supportare il telelavoro, tra cui ad esempio soluzioni per videoconferenze e riunioni online, spazi di lavoro virtuali, sicurezza informatica e formazione digitale. Le informazioni sul Digital Team Austria sono disponibili su un sito web, dove le PMI possono conoscere le aziende IT aderenti all'iniziativa ed entrare in contatto diretto con loro.

Analogamente, il Ministro dell'Innovazione italiano ha lanciato un'iniziativa denominata "Solidarietà digitale". Questa iniziativa prevede la creazione di un portale online dove le imprese (in particolare le PMI e i lavoratori autonomi) possono registrarsi per accedere ai servizi digitali gratuiti forniti dalle grandi aziende del settore privato in ambiti quali telelavoro, videoconferenza, accesso ai dati mobili e cloud computing, per consentire loro di far fronte alle restrizioni imposte alla circolazione e al lavoro.

Anche altri Paesi, tra cui Canada, Irlanda, Malesia, Messico e Portogallo, si sono attivati per facilitare l'accesso agli strumenti digitali. Lo strumento online del servizio nazionale canadese per l'impiego "Job Bank" ha adeguato il suo sito web e ha adattato i suoi servizi per sostenere i lavoratori e i datori di lavoro durante la pandemia di COVID-19, aggiungendo una nuova funzione che consente ai candidati di cercare in modo specifico opportunità di telelavoro. Enterprise Ireland, l'organizzazione governativa irlandese responsabile dello sviluppo e della crescita delle imprese nazionali nei mercati mondiali, ha creato una pagina web dedicata al COVID-19. La pagina web fornisce informazioni sul telelavoro e sull'accesso agli strumenti digitali. La Malaysia Digital Economy Corporation, istituita dal governo come parte della strategia digitale del Paese, offre un ampio elenco di soluzioni digitali per le PMI fornite dalle aziende tecnologiche malesi. II Messico ha annunciato il lancio del micro-sito "Together for Work" che fornisce consulenza e orientamento sul telelavoro, formazione a distanza, oltre a servizi di sostegno, consulenza legale e promozione aziendale. II governo portoghese, in collaborazione con le aziende tecnologiche, ha messo a disposizione una serie di strumenti gratuiti a supporto del telelavoro attraverso l'istituzione di un centro di contatto.

Infine, un esempio rilevante di un governo subnazionale proviene dalla provincia del Québec (Canada), che ha creato una directory pubblica per promuovere i rivenditori locali che offrivano modalità di acquisto online durante la fase più acuta della pandemia.

\section{Sostegno non finanziario alle PMI per l'adozione del telelavoro: campagne informative e formazione}

Le amministrazioni regionali e locali hanno avuto un ruolo primario nel condurre campagne informative e corsi di formazione sulle modalità di telelavoro e hanno facilitato l'accesso alla formazione professionale per le PMI durante l'emergenza di COVID-19. Ciò ha contribuito a garantire che i lavoratori potessero facilmente passare al telelavoro e avere anche l'opportunità di riqualificare, migliorare e ampliare le proprie competenze.

Diverse città hanno istituito sportelli dedicati a fornire informazioni sul telelavoro. Ad esempio, a Barcellona (Spagna), Barcelona Activa, l'agenzia di supporto alle imprese cittadine, gestisce una rete di centri di informazione che forniscono assistenza on-line e telefonica su questioni occupazionali rilevanti nell'ambito dell'epidemia di COVID-19, come i licenziamenti temporanei, le modalità di telelavoro, la prevenzione dei rischi sul lavoro e l'igiene sul posto di lavoro. Al 9 aprile 2020, Cibernàrium, il centro di formazione e diffusione tecnologica di Barcelona Activa, aveva ricevuto più di 5800 domande di iscrizione, triplicando il numero dei propri abbonamenti. A Braga (Portogallo) InvestBraga, l'agenzia municipale per lo sviluppo economico, e la sua filiale per l'innovazione Startup Braga hanno organizzato una serie di corsi online e webinar per aiutare le PMI a migliorare le proprie competenze digitali in settori quali e-commerce, telelavoro e videoconferenze. 
Alcune regioni hanno istituito organismi di supporto tecnico per la formazione e l'assistenza ai lavoratori nell'adozione del telelavoro. L'agenzia regionale per lo sviluppo economico dei Paesi Baschi (Spagna) ha lanciato Inplantalariak, ovvero un team di consulenti tecnologici che fornisce assistenza gratuita a lavoratori autonomi e microimprese con meno di dieci dipendenti nella ricerca di soluzioni per il telelavoro, il monitoraggio e la gestione a distanza, la sicurezza informatica e il commercio elettronico.

Infine diverse regioni hanno adottato misure per sostenere l'offerta didattica e la formazione a distanza, per garantire che i lavoratori possano accedere alle opportunità di sviluppo delle competenze durante il COVID-19. Un esempio di rilievo è dato dalla Regione dell'Occitania (Francia) che ha sviluppato un piano volto a "formare invece di licenziare" (former plutôt que licencier). II piano prevede 4 milioni di euro a sostegno delle persone in cerca di lavoro che desiderano continuare o intraprendere la formazione a distanza. In Francia, anche le Regioni dell'Île-de-France e della Normandia hanno disposto servizi di supporto per garantire che le organizzazioni che forniscono formazione possano continuare le loro attività online durante la pandemia di COVID-19.

Anche molte regioni in Italia hanno fatto passi in questa direzione. La Regione Autonoma Friuli Venezia Giulia, ad esempio, offre un sostegno agli enti pubblici preposti alla formazione professionale per rafforzare la loro capacità di erogare formazione a distanza, mentre la Liguria mette a disposizione un percorso integrato di politica attiva del lavoro, combinando l'e-learning con il sostegno al reddito per i disoccupati temporanei. I beneficiari possono anche richiedere un voucher per l'acquisto o il noleggio di tecnologie digitali. Entrambi i programmi citati sono finanziati dal Fondo Sociale Europeo. La Regione Abruzzo ha adottato il quadro di riferimento "Linee guida per l'utilizzo della formazione a distanza/e-learning" definito dalla Conferenza Italiana delle Regioni nel 2019, che consente l'apprendimento a distanza nelle attività di formazione professionale gestite dagli enti regionali.

A livello nazionale, un altro esempio è dato dalle attività svolte dall'Agenzia Turca per l'Occupazione (IŞKUR) per aumentare l'occupabilità dei giovani attraverso politiche attive del lavoro come la formazione sul posto di lavoro, la formazione professionale e la consulenza. Job Clubs Project in particolare, è un programma intensivo di consulenza professionale e occupazionale che fornisce supporto metodologico e motivazionale a gruppi target speciali come donne, giovani, disabili e disoccupati di lunga durata. Dopo lo scoppio dell'epidemia, i Job Clubs e altri servizi come l'orientamento degli studenti e la consulenza ai datori di lavoro hanno iniziato a essere offerti online, coinvolgendo chi cerca lavoro e chi lo offre attraverso i social media e altri canali online.

\section{Sostegno finanziario alle PMI per l'adozione del telelavoro: sovvenzioni e altri incentivi}

Durante la pandemia di COVID-19 diversi governi locali in tutta l'area OCSE, e non solo, hanno dimostrato di essere consapevoli delle sfide che le PMI devono affrontare nell'adozione del telelavoro e hanno messo in atto strumenti di policy dedicati. L'esempio più comune è la concessione di sovvenzioni per ridurre i costi degli investimenti IT sostenuti dalle micro e piccole imprese. Sebbene vi siano alcuni casi di politiche nazionali che rientrano in questa categoria, sono stati i governi regionali a ricoprire il ruolo più importante in questo ambito.

Vi sono diversi esempi a livello nazionale di offerta di sovvenzioni e accesso ai servizi digitali per le PMI. II governo giapponese ha introdotto aiuti economici per le PMI che utilizzano il telelavoro che possono arrivare a coprire fino al $50 \%$ dei costi per l'adozione di soluzioni informatiche, la creazione o la modifica di pratiche lavorative e la formazione dei lavoratori, ecc. In Spagna, il programma Acelera PYME fornisce sovvenzioni e prestiti per sostenere le PMI nel finanziamento di investimenti in attrezzature digitali o soluzioni di telelavoro. Al di fuori dell'OCSE, la Cina supporta le PMI nel cloud computing e nell'acquisto di tecnologia cloud e per il lavoro online come i software per la gestione dell'ufficio da remoto, l'home office, le videoconferenze, la formazione online, la R\&S collaborativa e l'e-commerce. 
Esempi di misure a livello subnazionale sono presenti in almeno sette Regioni italiane. La Puglia ha pubblicato un bando per l'attivazione di un "Piano di Innovazione Family friendly nelle PMl". Gli investimenti ammissibili alle sovvenzioni dovrebbero mirare alla de-standardizzazione dell'orario di lavoro (ad esempio attraverso un maggiore utilizzo di orari flessibili o del time banking) o allistituzione di nuove modalità lavorative, come il telelavoro. La Basilicata ha istituito un regime di sostegno alle imprese che adottano piani di telelavoro: le PMI, i lavoratori autonomi e i liberi professionisti possono ricevere aiuti fino al $70 \%$ della spesa sostenuta per l'attivazione di piani di telelavoro per i propri dipendenti. II Friuli Venezia Giulia ha lanciato un bando per la concessione di contributi ai lavoratori autonomi, alle cooperative e alle piccole imprese fino a 50 dipendenti per sostenere l'adozione di piani di telelavoro, con contributi a copertura di tutte le spese sostenute durante l'intero ciclo di vita dell'adozione del telelavoro. La Liguria ha istituito un sistema di voucher per le PMI che adottano piani di telelavoro: le agevolazioni coprono il $60 \%$ dell'investimento per la formazione e la tecnologia digitale. La Lombardia ha istituito un sistema pubblico a sostegno dell'adozione di piani di telelavoro, con contributi a copertura dei costi sostenuti per la formazione digitale e l'acquisto di tecnologie informatiche. II Molise ha pubblicato il bando pubblico "lo lavoro agile" per la promozione del telelavoro tra le PMI. Infine, il Piemonte ha ampliato un programma preesistente per il telelavoro, aumentandone il budget. Tutte le misure adottate dalle Regioni italiane attingono ai Programmi Operativi Regionali legati al Fondo Sociale Europeo (FSE) o al Fondo Europeo di Sviluppo Regionale (FESR).

Per promuovere il telelavoro tra le PMI, i Paesi Baschi hanno lanciato l'iniziativa "Industria Digitala" che sovvenziona tra il $25 \%$ e il $50 \%$ (a seconda delle dimensioni dellimpresa) delle spese sostenute per la consulenza digitale, la progettazione e l'acquisizione di hardware e software per promuovere il telelavoro. Questo programma è finanziato attraverso il FESR.

In Austria esiste dal 2019 il programma di finanziamento "Families! Friendly", con il quale la Camera del Lavoro della Stiria (AK) e l'Agenzia per la promozione delle imprese della Stiria (SFG) sostengono la creazione di posti di lavoro a distanza (e di luoghi di lavoro più inclusivi) nelle piccole imprese. II programma è stato ampliato grazie al nuovo programma "Telearbeit! Offensive". II programma si applica alle micro o piccole imprese con sede in Stiria. Gli investimenti ammissibili coprono le spese correnti per le tariffe telefoniche e internet, le attrezzature d'ufficio e commerciali, i telefoni cellulari, le infrastrutture presso la sede dell'azienda nonché il noleggio o il leasing di attrezzature (ad es. server). La quota dei costi coperti è dell' $80 \%$.

In Giappone il governo metropolitano di Tokyo ha offerto aiuti finanziari alle PMI e ad altre aziende per il passaggio al telelavoro. II sussidio coprirà le spese sostenute per i software e altre attrezzature necessarie per il telelavoro.

\section{Politiche per l'attrazione dei lavoratori a distanza e dei lavoratori nel settore digitale}

Negli ultimi anni, e quindi anche prima della pandemia, le politiche volte ad attirare telelavoratori e lavoratori nel settore digitale si sono diffuse in tutto il mondo. Questa sezione si propone di fornire una breve panoramica di queste esperienze così diversificate, che potrebbero acquisire nuovo slancio nel caso in cui il telelavoro di massa diventasse un fattore permanente.

Diverse città e stati degli Stati Uniti hanno visto il potenziale delle politiche di attrazione dei lavoratori a distanza anche prima della pandemia. Nel 2018, lo Stato del Vermont ha approvato il Remote Worker Grant Program per incoraggiare i professionisti interessati a trasferirsi nel Vermont. Nello stesso anno, un'altra giurisdizione degli Stati Uniti - la città di Tulsa, Oklahoma - ha lanciato un'iniziativa simile (Business Wire, 2018[66]). Entrambi i programmi forniscono ai lavoratori a distanza sovvenzioni per un rimborso fino a 10000 dollari. I loro obiettivi dichiarati includono la lotta contro lo spopolamento e l'attrazione di talenti altamente qualificati. Tra gennaio e settembre 2019, l'autorità di gestione del 
programma del Vermont ha assegnato circa 321000 dollari a 84 nuovi lavoratori a distanza (Kurrle et al., $\left.2019_{[67]}\right)$. Nel suo primo anno, Tulsa Remote ha ricevuto più di 10000 candidature da oltre 150 Paesi e da tutti i 50 stati degli Stati Uniti, accogliendo accolto a Tulsa quasi 100 partecipanti all'iniziativa. Nel suo secondo anno, Tulsa Remote sta più che raddoppiando questo numero attraverso la selezione di 250 persone che riceveranno il pacchetto di incentivi (Business Wire, 2019[68]).

Questo tipo di politiche può essere promosso anche dalle piccole città. A partire dal 2019, Wittenberge (19 000 abitanti) nel Brandeburgo (Germania), è sede di un'iniziativa pilota pubblico-privata volta ad attrarre lavoratori nei settori creativi e digitali. Mentre gli investitori privati forniscono le strutture lavorative (hanno ristrutturato un vecchio frantoio e lo hanno trasformato in uno spazio di co-working), il Comune mette a disposizione gli spazi abitativi (riqualificando le case sfitte e offrendo tariffe molto basse per gli affitti, oltre a pagare le spese per l'ufficio). Altre località della Germania orientale, la cui popolazione è in calo sin dalla riunificazione, hanno preso iniziative simili, come l'Alta Lusazia (Sassonia) e Eberswalde (Brandeburgo). Nel secondo semestre del 2020, 20 dei 60 candidati hanno avuto la possibilità di trasferirsi nello spazio di co-working di Wittenberge.

Gli spazi di co-working sono un'altra opportunità per promuovere il lavoro a distanza. Come riportato nel quadro del progetto pilota CoWordLand, istituito nel 2019 dalla Heinrich-Böll-Stiftung Schleswig-Holstein, una serie di regioni e città di tutta la Germania e di altri Paesi europei, dalla Francia alla Bulgaria, stanno sperimentando spazi di co-working nelle aree rurali come nuova soluzione per riqualificare gli edifici vuoti e combattere l'esodo giovanile.

I programmi start-up visa per l'avvio di un'impresa cercano di attirare talenti e imprese dall'estero. Si tratta di politiche nazionali di immigrazione che mirano ad attirare i fondatori di imprese stranieri offrendo procedure amministrative semplificate per la fornitura di visti d'ingresso e permessi di soggiorno, nonché, occasionalmente, incentivi fiscali e sostegno finanziario. Questa è stata una tendenza sin dal lancio di Start-up Chile nel 2010. Da allora molti programmi hanno seguito le sue orme in tutto il mondo: nel 2016, l'organizzazione no profit Startup Polonia ha registrato un numero enorme di iniziative dal Canada a Israele, dall'Italia alla Nuova Zelanda (Startup Poland, 2016 $\left.6_{[69]}\right)$. Nei suoi primi sette anni di attività, StartUp Chile ha accolto più di 1600 start-up (che hanno coinvolto 4500 imprenditori) da 85 Paesi, con una valutazione lorda del portafoglio di 1,4 miliardi di dollari e un miliardo di dollari di capitale raccolto.

Tuttavia non tutte le politiche pubbliche per attirare i lavoratori del settore digitale mirano a trasferirli sul territorio del Paese in questione, come dimostra il caso estone della residenza digitale (e-Residency). II programma definisce un'identità digitale emessa dal governo che consente la creazione a distanza di un'azienda con sede nell'UE, oltre ad accedere al contesto imprenditoriale e ai servizi digitali dell'Estonia. Sebbene non richieda una delocalizzazione, i benefici proposti hanno una chiara dimensione territoriale: secondo il governo estone i residenti digitali conducono affari con altre aziende locali, investono, viaggiano e, in alcuni casi, pagano le tasse nel Paese (Kaljulaid, 2018[70]). Nei suoi primi cinque anni di vita, 62000 persone da tutto il mondo sono divenuti residenti digitali in Estonia e hanno fondato oltre 10100 aziende che danno lavoro a circa 1700 persone (The Baltic Times, 2019[71]). Gli intervistati nell'ambito di un sondaggio somministrato dal personale dallo staff di e-Residency durante la pandemia di COVID-19 hanno elogiato la convenienza del programma per i lavoratori a distanza e gli imprenditori senza sede fisica che possono gestire i loro affari interamente online senza la presenza e i limiti dei confini. Questa modalità aiuta quindi gli imprenditori e le loro aziende ad essere digitalmente resilienti anche in un momento in cui le frontiere sono chiuse e i viaggi internazionali sono interdetti, la maggior parte delle persone lavora da casa, e vi sono lockdown generalizzati per tutti i beni ad eccezione di quelli essenziali (Brown, 2020[72]).

Riassumendo, mentre le politiche quadro come i programmi di residenza digitale e gli schemi sui visti sono appannaggio esclusivo dei governi nazionali, le autorità locali subnazionali come le regioni, le città e anche i comuni di dimensioni ridotte possono dare vita a programmi di attrazione, la cui offerta (strutture lavorative o abitative, incentivi fiscali o altri incentivi economici, ecc.) può dipendere dalle risorse presenti a livello locale. Politiche di questo tipo hanno una chiara dimensione territoriale, ma il loro impatto effettivo sui flussi 
dei lavoratori è variabile. Le evidenze che emergono dai progetti locali sono per lo più limitate, soprattutto a causa della loro recente introduzione, mentre i programmi strutturali adottati dai governi nazionali (ad esempio Start-up Chile, la residenza digitale in Estonia) in anni relativamente recenti hanno già mostrato risultati promettenti.

\section{Politiche a sostegno delle imprese home-based}

II telelavoro non riguarda solo i lavoratori dipendenti ma anche quelli autonomi. L'esperienza delle politiche pre-COVID per le imprese home-based può fornire ai governi spunti utili per sostenere i lavoratori autonomi e i liberi professionisti che sono passati al telelavoro solo negli ultimi mesi o che stanno pianificando di farlo.

Un rapporto OCSE-UE del 2018 offre un ritratto delle imprese home-based, definite come imprese situate in abitazioni piuttosto che in uffici o altri locali commerciali. Questo tipo di società rappresenta poco più della metà delle imprese private, e mentre la maggior parte delle imprese home-based sono ditte individuali, fino al $40 \%$ ha dipendenti aggiuntivi. La riduzione dei costi di gestione e le migliori prospettive di conciliazione vita-lavoro sono le ragioni più comuni per avviare un'impresa di questo tipo. Mentre alcune imprese home-based hanno un potenziale di crescita, molte cercano di rimanere piccole per mantenere la sede nella propria abitazione. II lavoro da casa sembra essere più comune tra le lavoratrici autonome che tra gli uomini (Reuschke and Domecka, 2018[73]).

La comunità imprenditoriale home-based è molto diversificata. Le sfide che devono affrontare possono variare a seconda del settore interessato, della posizione geografica e delle caratteristiche del proprietario dell'azienda. Le politiche pubbliche possono favorire la creazione e la crescita di imprese home-based in diversi modi, come dimostrato da una serie di esperienze da tutto il mondo.

Un'importante area di policy comprende l'accesso alla consulenza aziendale, al tutoraggio e alle reti imprenditoriali. Le misure in questo campo spesso vanno di pari passo con l'offerta di spazi di lavoro e di incontro a prezzi accessibili per un uso flessibile da parte di una serie di potenziali imprenditori homebased con esigenze specifiche (ad esempio, assistenza all'infanzia, sviluppo delle competenze e disabilità). Ad esempio, la Città di Milano concede voucher per i lavoratori del settore digitale che si trasferiscono in uno spazio pubblico di co-working, mentre la Camera di Commercio di Vienna offre un supporto aziendale individuale e l'accesso gratuito agli spazi di incontro. Allo stesso modo, la Città di Barcellona finanzia la Almogàvers Business Factory con lo scopo di fornire formazione individuale e di gruppo e consulenza aziendale. In Australia, il programma Entrepreneurship Facilitators porta il tutoraggio e la consulenza aziendale direttamente nelle case delle persone, concentrandosi su aree ad alta disoccupazione giovanile e di lunga durata in età matura.

I decisori politici possono anche rimuovere gli ostacoli alle attività imprenditoriali svolte da casa in ambito normativo, al momento dell'elaborazione delle policy e in materia di progettazione urbana, adeguando i sistemi di classificazione degli edifici monofunzionali, le regole sui contratti di locazione e la tassazione degli immobili. Come dimostrato dai Paesi Bassi, che da anni sperimentano con successo la pianificazione duale degli edifici, è probabile che uno spostamento della cultura dell'edilizia abitativa e della pianificazione verso il sostegno e l'incoraggiamento del lavoro da casa stimoli dinamiche imprenditoriali tra gli inquilini e i proprietari. Anche le associazioni edilizie possono essere un partner proattivo nel sostenere la creazione di imprese home-based, in particolare nelle aree marginali e tra le fasce svantaggiate. Ad esempio, nel 2013, nel Regno Unito, Cube Housing ha fornito ai propri inquilini 15 sovvenzioni del valore di 1000 sterline (circa 1110 euro) per l'avvio di start-up.

Le politiche e i programmi a sostegno della conciliazione tra lavoro e vita privata sono rilevanti anche per molti imprenditori che operano da casa. Nel 2017, la Commissione Europea ha lanciato l'iniziativa "WorkLife Balance Initiative for Working Parents and Carers", incoraggiando un migliore utilizzo dei fondi UE per 
migliorare i servizi di assistenza all'infanzia e quella a lungo termine. In questo modo, l'iniziativa mirava ad aumentare la partecipazione al mercato del lavoro, anche attraverso il lavoro autonomo. Un caso pratico in questo senso è il progetto Caboodle Collaborative Workspace Project a Bristol, Regno Unito, rivolto agli imprenditori che lavorano da casa che hanno bisogno di assistenza all'infanzia. Lanciato nel 2017, questo programma offre sessioni "pop-up" di co-working in collaborazione con un asilo nido in loco, consentendo ai genitori e agli assistenti all'infanzia di lavorare fianco a fianco mentre i bambini sono accuditi nelle immediate vicinanze.

Gli schemi di microcredito amministrati attraverso organizzazioni locali e comunitarie possono essere particolarmente utili per le aziende che operano da casa. La US Small Business Administration riferisce che praticamente tutte le aziende che ricevono un prestito inferiore a 20000 dollari sono home-based. II finanziamento peer-to-peer e il crowdfunding sono altre opzioni importanti. Ad esempio, la Città di Milano gestisce un programma per start-up di innovazione sociale, integrando i fondi raccolti attraverso campagne di crowdfunding con denaro pubblico. A partire dal 2017, sono state sostenute diverse imprese homebased. II programma UE per l'occupazione e l'innovazione sociale mira anche a migliorare l'accesso ai finanziamenti per le imprese che operano da casa. Lanciato nel 2014, promuove la disponibilità e l'accessibilità alla microfinanza per i gruppi vulnerabili e le microimprese e facilita l'accesso ai finanziamenti per le imprese sociali.

È inoltre necessario migliorare l'accesso alle informazioni sulle normative locali e nazionali che si applicano alle imprese home-based. Alcuni Paesi e Città hanno prodotto guide dedicate alle imprese che operano da casa, come Alberta e Ottawa (Canada), Scozia (Regno Unito) e Victoria (Australia).

Infine, i decisori politici possono migliorare la base di conoscenze sulle attività commerciali home-based. Mentre i registri delle imprese e i dati delle indagini sulla forza lavoro possono fornire alcune informazioni su questo gruppo, la maggior parte delle evidenze provengono da sondaggi. L'ampliamento della base di dati potrebbe contribuire a rendere più visibile questo fenomeno. 


\section{Messaggi principali per i responsabili politici}

\section{L'impatto non neutrale del telelavoro su persone, territori e imprese}

La pandemia di COVID-19 ha dimostrato come che il telelavoro su larga scala e su base permanente ha un impatto non neutrale sulle persone (datori di lavoro, dipendenti), sui territori (aree urbane, semidense e rurali) e sulle imprese (grandi imprese e PMI), anche nel breve periodo. Lo dimostra il fatto che decisori politici, associazioni imprenditoriali, sindacati, giuristi e urbanisti di tutto il mondo stiano osservando con massima attenzione questo fenomeno.

Diversi gruppi di lavoratori sono esposti alle opportunità e alle minacce derivanti dal telelavoro in modi differenti: lavoratori stabili e temporanei, dipendenti e autonomi, genitori e non, maschi e femmine, senior e junior, ad alto e a basso livello di reddito/qualifica, residenti e transfrontalieri, locali e immigrati, ecc. ciascuno di questi gruppi ha una diversa prospettiva sulla materia. Secondo le indagini condotte in tutto il mondo negli ultimi mesi, i dipendenti hanno dimostrato una generale disponibilità a proseguire il telelavoro dopo l'emergenza sanitaria, e molti grandi datori di lavoro del settore digitale e non solo stanno valutando la possibilità di adottare piani di telelavoro su larga scala. Tuttavia, i rapporti indicano che durante la pandemia i dipendenti hanno lavorato molto più a lungo del solito - uno dei tanti aspetti che richiedono una transizione graduale verso il telelavoro di massa.

La disponibilità dei dipendenti al telelavoro riflette le differenze di reddito: la trasformazione in corso non è neutrale, poiché rischia di amplificare le disuguaglianze preesistenti. Le competenze digitali fanno la differenza nel consentire l'accesso al telelavoro, e le istituzioni pubbliche possono aiutare le persone, le imprese e i territori a recuperare il ritardo, fornendo informazioni e formazione, anche in collaborazione con gli attori privati (ad esempio attraverso campagne di sensibilizzazione promosse dal governo e realizzate dalle camere di commercio o da altre reti di imprese sul territorio). Oltre alle competenze digitali, il telelavoro richiede collaborazione e capacità di gestione da parte sia del dipendente che del datore di lavoro, nonché capitale organizzativo aziendale e disponibilità di infrastrutture informatiche di alta qualità.

Le aziende non sono egualmente attrezzate per affrontare uno scenario di telelavoro di lunga durata, e tale propensione varia a seconda delle loro dimensioni, del settore e dell'ubicazione. Mentre la gran parte delle imprese più grandi ha una maggiore familiarità con il telelavoro e può beneficiare delle economie di scala in misura più significativa, le PMI spesso non hanno una conoscenza diretta di questa modalità e mancano del know-how, delle risorse finanziarie e delle competenze necessarie per adattarvisi. Le politiche possono aiutare le PMI a colmare il divario digitale in termini di investimenti. Tuttavia, alcuni settori e mansioni sono più adatti al telelavoro rispetto ad altri: il lavoro fisico, che svolge un ruolo primario in molti settori essenziali, spesso non è sostituibile.

Quando vigono misure di contenimento, i lavoratori altamente qualificati sono meno esposti a minacce per l'occupazione, poiché è più probabile che il loro lavoro si possa svolgere a distanza rispetto a quello dei lavoratori poco qualificati. Pertanto le regioni in cui la loro incidenza è più elevata sono relativamente meno vulnerabili. Quando il telelavoro rappresenta l'unica possibilità per garantire la continuità aziendale, le ditte 
situate in aree con buone infrastrutture digitali beneficiano di un importante vantaggio competitivo rispetto a quelle situate in regioni meno performanti. Infine, le regioni con una composizione settoriale diversificata possono essere più preparate alla digitalizzazione e quindi vi è un'alta probabilità che una quota maggiore di posti di lavoro a livello locale sia adatta al telelavoro.

\section{Nuove conoscenze sul telelavoro e percorsi futuri}

\section{La necessità di una transizione lungimirante e monitorata}

La crisi pandemica ha accelerato un processo di digitalizzazione preesistente e dirompente, non solo tra le aziende ma all'interno della società nel suo complesso: e-commerce, apprendimento online e assistenza sanitaria a distanza, proprio come il telelavoro, sono esempi pratici della trasformazione in atto. Per questo motivo, il passaggio al telelavoro può essere concepito come un processo di lunga durata che contribuirà a rimodellare il futuro del lavoro, piuttosto che come un'eccezione a breve termine che avrà fine quando le politiche di distanziamento sociale non saranno più necessarie per affrontare la crisi sanitaria. In quest'ottica, i responsabili politici potrebbero cercare di promuovere con attenzione il telelavoro in un modo che vada a beneficio sia dell'economia che della società nel suo complesso, nel lungo periodo.

Tuttavia, vi sono evidenze contrastanti riguardo all'impatto del telelavoro su una serie di aspetti chiave, tra cui la produttività e la soddisfazione dei lavoratori. In effetti, lo stesso telelavoro non è più quello che conoscevamo prima della pandemia, data la sua marcata crescita dimensionale e il suo nuovo ruolo potenziale di pratica strutturale a lungo termine. Pertanto, i responsabili politici potrebbero impegnarsi a costruire una nuova base di conoscenze e a monitorare le tendenze future, promuovendo una più ampia raccolta di dati sulle pratiche di telelavoro per garantire un cambiamento senza intoppi e sfruttare al meglio questa modalità. Un approccio "test-and-learn", basato sull'identificazione di obiettivi e soluzioni di policy, sul monitoraggio e sulla discussione dei progressi compiuti in consultazione con le parti sociali, potrebbe essere un'opzione meritevole di considerazione.

\section{L'importanza di un sistema di regole agile e del dialogo con i portatori d'interesse}

Le nuove iniziative sul telelavoro dovranno essere coerenti con la più ampia regolamentazione in materia di lavoro. La consapevolezza delle condizioni quadro sarà fondamentale per calibrare le risposte di policy, consentendo ai responsabili politici di scegliere la giusta combinazione di decisioni tra l'introduzione di nuovi atti legislativi, la modifica di leggi preesistenti e l'emanazione di regolamenti non vincolanti. I codici del lavoro possono essere adattati per consentire questa pratica e proteggere adeguatamente i diritti dei dipendenti e dei datori di lavoro, tenendo conto delle sfide derivanti dal telelavoro che riguardano, tra l'altro, la conciliazione tra lavoro e vita privata, la produttività, l'accesso universale alla formazione, la privacy e le opportunità di sviluppo professionale. II telelavoro solleva anche nuove questioni suscettibili di essere affrontate a livello normativo, come il diritto alla disconnessione per evitare che si creino commistioni tra lavoro e vita personale. Le politiche potrebbero anche tenere conto di eventuali ricadute del telelavoro sullo sviluppo urbano e rurale, sull'ambiente, sull'economia e sulla società nel suo complesso, per non parlare del suo potenziale contributo a un più consistente utilizzo di pratiche digitali da parte delle PMI. L'adozione di nuove normative trarrebbe grande beneficio da un ampio dialogo con le parti sociali e gli altri soggetti interessati: in questo contesto, data la pluralità di interessi in gioco, sono particolarmente raccomandati i processi di elaborazione delle politiche generati dal basso.

La crisi pandemica ha dimostrato come le strutture decisionali agili abbiano maggiori probabilità di adattarsi e sopravvivere ai cambiamenti e alle crisi, mentre le organizzazioni rigide sono più vulnerabili. Lo stesso concetto può essere applicato alle politiche pubbliche. Data la fluidità delle trasformazioni attualmente in corso, le politiche non dovrebbero essere viste come qualcosa di immutabile, bensì come un processo di adattamento costante. I decisori politici potrebbero quindi optare per una regolamentazione agile, aperta 
all'innovazione e ai cambiamenti. I cosiddetti regulatory sandbox (iniziative speciali adottate dal legislatore per condurre esperimenti normativi in un ambiente controllato) potrebbero contribuire, attraverso soluzioni innovative pilota, ad aprire la strada a cambiamenti duraturi. Allo stesso tempo, orari di lavoro misti, che combinano il lavoro in ufficio e quote crescenti di lavoro a distanza, potrebbero contribuire a una transizione fluida.

\section{II telelavoro potrebbe contribuire a promuovere l'equità e l'uguaglianza, se accompagnato da interventi pubblici complementari}

II telelavoro potrebbe essere un potente strumento per promuovere l'equità e l'uguaglianza, generando opportunità per tutte le persone, i territori e le aziende e contrastando le disparità preesistenti. Inoltre, questo strumento potrebbe essere utilizzato in modo strategico a beneficio della società nel suo insieme. II telelavoro interessa diversi gruppi di lavoratori in modi differenti. Rispetto ai lavoratori dipendenti, i lavoratori autonomi possono organizzare il proprio lavoro come meglio credono, ma devono anche sostenere i costi degli investimenti. Le politiche adottate in passato a sostegno delle imprese home-based, compresi gli strumenti su misura per l'accesso al credito e la fornitura di servizi condivisi, possono aiutare a dare risposte alle sfide specifiche affrontate dai lavoratori autonomi che lavorano da casa. II telelavoro potrebbe amplificare le disuguaglianze tra donne e uomini in mansioni come le mansioni domestiche e l'assistenza all'infanzia. Diverse ricerche hanno dimostrato che la violenza domestica è aumentata durante questa crisi. Politiche e servizi sociali complementari contribuirebbero a rendere il telelavoro più sostenibile e accessibile a tutti. I giovani lavoratori e i tirocinanti sono un altro gruppo potenzialmente svantaggiato, poiché un passaggio al telelavoro di massa potrebbe ostacolare la loro capacità di ampliare le loro reti relazionali e di apprendere dai colleghi più esperti. Anche i lavoratori transfrontalieri potrebbero essere danneggiati, qualora le normative fiscali non tengano conto delle esigenze derivanti dal telelavoro a lungo termine all'estero.

Più in generale, i lavoratori poco qualificati e temporanei sono tra i meno inclini a beneficiare del telelavoro, poiché di solito operano in ambiti che non si prestano al lavoro a distanza quali i servizi diretti al cliente, l'industria manifatturiera e l'agricoltura. In questo contesto, le politiche potrebbero fornire opportunità di rafforzamento delle competenze e migliorare il costo-opportunità di lavorare nei settori essenziali. A prescindere dal gruppo di appartenenza, a tutti i telelavoratori sarà richiesto di migliorare le proprie competenze digitali, gestionali e organizzative. I governi nazionali, regionali e locali potrebbero svolgere un ruolo importante nel promuovere e sovvenzionare la formazione.

\section{Le politiche possono aiutare a rendere il telelavoro accessibile a tutte le aziende}

Una diffusione duratura e di massa del telelavoro avrebbe un impatto diversificato sulle aziende a seconda delle loro dimensioni, del settore e della loro posizione geografica. Le PMI devono affrontare costi sproporzionatamente più elevati per gli investimenti in tecnologia digitale e formazione, e hanno meno probabilità di sfruttare le economie di scala rispetto alle imprese più grandi. Inoltre, alcuni settori e mansioni sono più adattabili al telelavoro rispetto ad altri, così come lo sono le imprese situate in aree con reti ad alta velocità.

Le politiche possono contribuire a rendere il telelavoro accessibile a tutte le aziende creando condizioni di parità e compensando gli svantaggi competitivi. Sovvenzioni e altri incentivi finanziari per le PMI che adottano piani di telelavoro possono contribuire a ridurre i costi di investimento, mentre l'offerta di formazione e la creazione di un'infrastruttura digitale diffusa potrebbero contribuire a garantire che tutte le imprese competano su un piano di parità. Le politiche possono anche ricoprire un ruolo chiave nel promuovere strutture gestionali agili, più adattabili alle mutate circostanze, al fine di aumentare la resilienza delle imprese. Gli incentivi fiscali potrebbero essere indirizzati alle aziende che soddisfano i criteri di flessibilità e di benessere aziendale, come la promozione del telelavoro, le politiche a favore della famiglia 
o altre pratiche che favoriscono una migliore conciliazione tra lavoro e vita privata e l'inclusione di categorie svantaggiate.

\section{Le regioni in ritardo di sviluppo possono trasformare il telelavoro di massa in un'opportunità}

Infine, il telelavoro su larga scala potrebbe comportare una parziale inversione dei flussi di lavoratori tradizionali dalle aree urbane verso quelle semi-dense e rurali. I posti di lavoro effettuabili a distanza hanno maggiori probabilità di interessare le aziende i cui locali sono situati in aree urbane. Con il lavoro da casa che sta diventando una prassi comune e la vicinanza al posto di lavoro che vede ridimensionato il proprio valore, i telelavoratori sono più propensi a trasferirsi in zone a densità intermedia o addirittura in zone scarsamente popolate che offrono un maggiore benessere e costi inferiori. In questo caso la disponibilità di un accesso a internet ad alta velocità sarebbe un fattore chiave nel determinare la capacità dei territori di attirare e trattenere i lavoratori a distanza e altri lavoratori del settore digitale.

I responsabili politici regionali e locali possono svolgere un ruolo attivo in questo senso. Oltre a investire nelle infrastrutture IT, possono attuare una serie di strumenti, tra cui sovvenzioni, agevolazioni fiscali e altri incentivi economici, per attrarre lavoratori senza una sede fissa. L'offerta di strutture di co-working, affitti a basso costo per gli alloggi e l'accesso alle comunità imprenditoriali e ai servizi pubblici possono integrare tali programmi. Inoltre le regioni e le comunità locali potrebbero cogliere l'opportunità di promuovere la diversificazione della loro base industriale, aumentando la loro propensione alla digitalizzazione e ai fenomeni ad essa associati, compreso il telelavoro. Molte regioni UE stanno facendo leva sul Fondo Sociale Europeo e sul Fondo Europeo di Sviluppo Regionale come fonti di finanziamento per i programmi di attrazione. Allo stesso tempo, le città dovrebbero prepararsi a perdere il loro vantaggio competitivo intrinseco in termini di attrattività, che finora ha prevalso su questioni importanti come la bassa qualità $e$ l'alto costo degli alloggi, il sovraffollamento e l'inquinamento atmosferico.

II Box 1 delinea una serie di principi guida e le relative raccomandazioni di policy che potrebbero aiutare a promuovere un modello di telelavoro sostenibile per persone, territori e aziende.

\section{Box 1. Principi guida e raccomandazioni di policy per una transizione graduale verso un} modello di telelavoro sostenibile per persone, territori e aziende

I seguenti principi guida potrebbero contribuire a promuovere una transizione graduale verso il telelavoro su larga scala e su base permanente:

a. Adottare un approccio olistico nell'ideazione delle politiche per il telelavoro. Una diffusione persistente e su vasta scala del telelavoro potrebbe influire profondamente sui mercati del lavoro locali nelle aree urbane e rurali, sui modelli residenziali e di mobilità, così come sulla società nel suo insieme. Questi impatti non sono ancora del tutto prevedibili. Una prospettiva multidisciplinare e a lungo termine nell'ideazione delle politiche contribuirebbe a declinare queste dinamiche trasformative a seconda delle opportunità di sviluppo locale.

b. Promuovere equità e inclusione tra i gruppi sociali, le imprese e i territori. II telelavoro non è accessibile in modo uniforme per le diverse tipologie di lavoratori, di territori (es. aree urbane e rurali) e di imprese (es. PMI e grandi aziende) e ha effetti diversificati su ciascuno di essi. Le misure in materia di telelavoro potrebbero contribuire a creare condizioni di parità, a patto che si mettano in atto politiche sociali complementari.

c. Dare la priorità agli obiettivi di natura collettiva. Una diffusione su larga scala del telelavoro potrebbe contribuire a ridurre il traffico urbano e le emissioni di $\mathrm{CO} 2$, favorire lo sviluppo rurale, promuovere l'uguaglianza di genere e perseguire altri obiettivi di sviluppo sostenibile. Gli 
interventi potrebbero dare la priorità a soluzioni vantaggiose per la collettività e attenuare le esternalità negative, come la drastica riduzione della domanda che potrebbe ripercuotersi sui negozi e i fornitori di servizi situati in prossimità degli uffici.

d. Fornire le condizioni quadro abilitanti a livello nazionale e locale. La presenza di un quadro regolamentare agile e di servizi pubblici locali efficaci (in settori quali l'assistenza all'infanzia e agli anziani), così come di reti internet ad alta velocità, competenze digitali e un ambiente online protetto sono requisiti necessari per rendere il telelavoro accessibile e sicuro per tutti.

e. Creare una nuova base di conoscenza. Date le caratteristiche inedite del telelavoro su vasta scala, è importante produrre nuovi dati e metriche per informare le decisioni politiche. Gli effetti dovrebbero essere monitorati nel tempo per evitare conclusioni non obiettive e tenere conto della molteplicità di fattori che influiscono sulle persone, le aziende e i territori. Aspetti quali la raccolta dei dati e l'adattabilità alle condizioni di contesto dovrebbero essere tenuti in conto sin dalla fase di ideazione delle politiche, prevedendo sistematicamente forme continue di monitoraggio e valutazione.

Sulla base dei principi sopra definiti, le seguenti raccomandazioni potrebbero sostenere i responsabili politici nazionali, regionali e locali nel promuovere un modello di telelavoro sostenibile e vantaggioso per tutti i gruppi sociali, i territori e le imprese:

a. Adottare un approccio olistico nella progettazione di politiche per il telelavoro:

- Favorire il dialogo con le parti sociali e gli altri attori socio-economici a livello locale per identificare l'insieme dei bisogni percepiti (equilibrio tra lavoro e vita privata dei dipendenti, produttività dell'azienda, tutela degli investimenti in immobili commerciali, mobilità urbana, ecc.) e cogliere ogni ricaduta a vantaggio della società (ad esempio, minori emissioni di CO2 dovute alla riduzione degli spostamenti da e verso il posto di lavoro, miglioramento del benessere, ecc.) che potrebbe derivare dal telelavoro di massa. La consapevolezza della gamma di interessi ed esigenze in gioco aiuterebbe i responsabili politici a individuare soluzioni che sfruttino le esternalità positive a livello sociale e compensino le perdite individuali.

- Abbracciare un approccio di policy adattivo, basato sulla sperimentazione, sul monitoraggio dei dati e, quando necessario, sull'aggiornamento delle misure. In effetti, il telelavoro di massa e duraturo è una nuova tendenza trasversale il cui impatto non è del tutto prevedibile e richiede flessibilità.

b. Promuovere l'equità e l'inclusione:

- Sviluppare servizi pubblici avanzati su base territoriale e incentivare gli investimenti delle imprese per garantire un accesso equo al telelavoro nei mercati del lavoro locali (ad esempio, asili nido, strutture di assistenza all'infanzia, baby-sitting e altri strumenti complementari a sostegno dei lavoratori-genitori). Prendere in considerazione la sperimentazione di pratiche speciali nel campo dell'edilizia popolare, come le soluzioni abitative intergenerazionali, a beneficio dei telelavoratori con compiti di assistenza all'infanzia o degli anziani.

- Proporre il telelavoro come un'opzione complementare, piuttosto che come un puro sostituto del lavoro tradizionale. Si raccomanda di promuovere un corretto equilibrio tra telelavoro e modalità più tradizionali, in modo da sfruttare al meglio i benefici del telelavoro $\mathrm{e}$ ridurre al minimo alcune sue ripercussioni negative. Soprattutto, le interazioni fisiche vanno salvaguardate.

- Assicurarsi che il telelavoro non riduca le opportunità di formazione sul posto di lavoro e di sviluppo professionale, specie per i lavoratori meno qualificati e temporanei, prevedendo indagini periodiche e creando sistemi di monitoraggio ad hoc. 
- Mettere a punto schemi di sostegno e monitoraggio per ridurre $i$ rischi di violenze domestiche nel contesto del telelavoro su base permanente e garantire che gli osservatori sulla violenza contro le donne siano preparati a controllare questo aspetto.

- Incoraggiare le imprese a continuare ad offrire opportunità di formazione a studenti e giovani in un contesto di telelavoro di massa, in modo da consentire alle prime di attrarre nuovi talenti e ai secondi di acquisire esperienza pratica e fare un passo in avanti verso il mercato del lavoro. Più in generale, un mercato del lavoro in costante evoluzione rende l'offerta di formazione una priorità per tutte le fasce d'età.

- Assicurare che il quadro fiscale e normativo per i lavoratori frontalieri non comporti alcuna ripercussione negativa in caso di passaggio al telelavoro.

- Sostenere i lavoratori autonomi e le microimprese nel passaggio al telelavoro, ricordando che questo modello non si applica ai soli lavoratori dipendenti. Le politiche per le imprese homebased adottate in tutto il mondo prima della pandemia di COVID-19 possono offrire utili spunti di riflessione. Le opzioni includono, tra le altre cose, la fornitura di spazi di co-working a prezzi accessibili, l'erogazione di formazione e servizi a domicilio, l'eliminazione degli ostacoli alle imprese home-based nei regolamenti e nella progettazione urbana, l'offerta di programmi per la conciliazione tra lavoro e vita privata, programmi di microcredito gestiti da organizzazioni su base comunitaria.

c. Dare la priorità agli obiettivi di natura collettiva:

- Intensificare gli sforzi per comprendere, misurare e sensibilizzare l'opinione pubblica sulle ricadute ambientali e sociali del telelavoro di massa, al fine di aumentarne l'accettazione sociale.

- Prevedere meccanismi di compensazione quando gli interessi individuali risultano danneggiati dal telelavoro, ad esempio offrendo sostegno finanziario temporaneo, incentivi a trasferirsi o a iniziare a vendere online per i rivenditori e i fornitori di servizi situati in prossimità degli uffici, che possono subire una riduzione della domanda.

- Sostenere la conciliazione tra lavoro e vita privata per garantire la sostenibilità e la resilienza nei mercati del lavoro locali, incoraggiando il telelavoro volontario e l'organizzazione flessibile del lavoro, le politiche a favore della famiglia e le pratiche di welfare aziendale. Questo obiettivo potrebbe essere raggiunto attraverso campagne di sensibilizzazione, la promozione delle migliori pratiche e incentivi fiscali.

- Stimolare una discussione tra le parti sociali sull'opportunità di introdurre un diritto alla disconnessione e favorire accordi collettivi o altre soluzioni condivise a questo proposito, al fine di compensare i rischi legati a una mancata conciliazione tra lavoro e vita privata per $\mathrm{i}$ dipendenti.

d. Fornire le condizioni quadro abilitanti:

- Assicurare che il quadro giuridico consenta il telelavoro e le opportunità in esso insite per tutti. Prendere in considerazione la sperimentazione di normative agili (norme non vincolanti, sandbox normativi, ecc.) e l'aggiornamento di quelle preesistenti (ad es. codici del lavoro). Assicurare che il quadro giuridico possa essere adattato ai cambiamenti delle macrotendenze.

- Incentivare la formazione dei lavoratori e dei manager per potenziare le competenze digitali, collaborative e organizzative necessarie per il telelavoro. Questi moduli potrebbero anche essere integrati nelle attività di formazione nell'ambito delle politiche attive del mercato del lavoro. 
- Incentivare gli investimenti per potenziare le infrastrutture IT nelle aree rurali e svantaggiate, anche promuovendo partenariati pubblico-privato con aziende specializzate, come i fornitori di internet via satellite, che possono raggiungere i residenti delle aree rurali o suburbane dove non sono disponibili connessioni internet via cavo o in fibra ottica. Le tecnologie ibride fisso-mobile (FWA) sono un'altra opzione che vale la pena di esplorare, considerando le loro prestazioni più elevate: I'obiettivo in questo caso sarebbe quello di portare le infrastrutture 4G (5G nel prossimo futuro) in aree remote che gli operatori privati non hanno alcun incentivo economico a coprire con tecnologie full-fibre (FTTH, "fibre-to-the-home"). La misura e velocità con cui il telelavoro può essere incoraggiato e adottato in modo più ampio possono dipendere dalla qualità e dall'affidabilità delle telecomunicazioni e di altre infrastrutture nelle aree non metropolitane e rurali.

- Prendere in considerazione l'istituzione di schemi di sostegno finanziario per favorire l'adozione del telelavoro da parte delle PMI, attenuando i costi sproporzionatamente più elevati che esse sostengono per adottare questa modalità rispetto alle imprese più grandi. Idealmente, gli schemi finanziari potrebbero coprire tutte le voci di costo, come ad esempio: consulenza da parte di professionisti certificati per la progettazione di piani di telelavoro; acquisto, installazione e assistenza di software, hardware e reti informatiche, sia in ufficio che a casa; strumenti di sicurezza informatica; formazione digitale per dipendenti e manager, ecc. Le regioni UE potrebbero prendere in considerazione la possibilità di sfruttare il Fondo Sociale europeo e il Fondo Europeo di Sviluppo Regionale per istituire incentivi e altri programmi finanziari per promuovere l'adozione del telelavoro tra le PMI.

- Considerare l'adozione di piani di attrazione e fidelizzazione dei lavoratori digitali e a distanza, offrendo un mix di incentivi basati sulle risorse locali. Le pratiche internazionali suggeriscono una varietà di opzioni, tra cui agevolazioni fiscali, sovvenzioni, fornitura di spazi di co-working, affitti per l'alloggio accessibili, integrazione in reti imprenditoriali, ecc.

e. Creare una nuova base di conoscenza:

- Includere la variabile del telelavoro negli schemi di rilevazione dei dati sull'occupazione. Le opzioni includono, tra le altre cose, l'aggiornamento dei sistemi statistici del mercato del lavoro a livello nazionale e regionale, l'introduzione di nuovi protocolli per la trasmissione dei dati sull'occupazione da parte delle imprese e la conduzione di indagini periodiche per monitorare l'opinione dei telelavoratori e dei loro datori di lavoro. Tra i nuovi indicatori da adottare vi sono, tra gli altri, la previsione di clausole sul telelavoro nei contratti di lavoro, il tipo di contratto di telelavoro e il rapporto tra le ore di telelavoro e il totale delle ore lavorate.

- Informare le politiche con dati e misurazioni aggiornate che riflettano la nuova portata e le implicazioni rilevanti a livello sociale, territoriale e aziendale del telelavoro di massa (frequenza di utilizzo tra i lavoratori, le regioni, i settori e le varie classi di dimensione aziendale, l'impatto sulle competenze locali e sull'occupazione, la soddisfazione dei dipendenti, le variazioni dei salari, ecc.). II monitoraggio di indicatori quantitativi (ad esempio produttività, risparmi/costi per le imprese e i lavoratori) e qualitativi (equilibrio tra lavoro e vita privata, fiducia e soddisfazione dei dipendenti e dei datori di lavoro) potrebbe migliorare i processi decisionali. Oltre agli indicatori relativi al lavoro, i sistemi di monitoraggio potrebbero rilevare altri aspetti, ad esempio le variazioni delle emissioni di $\mathrm{CO} 2$ nelle aree urbane dovute alla riduzione degli spostamenti da e verso il posto di lavoro. 


\section{Allegato A. Una rassegna delle politiche nazionali, regionali e locali per il telelavoro}

Questa sezione fornisce una raccolta di misure per il telelavoro adottate dai responsabili politici nazionali, regionali e locali prima e, nella maggior parte dei casi, durante la pandemia di COVID-19. Le politiche mappate coprono 26 Paesi e 29 Regioni e Città di 11 Paesi.

\section{Politiche nazionali per il telelavoro}

\section{Australia}

- Per mantenere i lavoratori in sicurezza e prevenire la diffusione del COVID-19, il governo federale australiano ha incoraggiato i dipendenti e i datori di lavoro a lavorare insieme per trovare le soluzioni più vantaggiose e praticabili che si adattino ai loro luoghi di lavoro e al loro personale.

- Le leggi australiane sulla salute e la sicurezza sul lavoro richiedono alle aziende di garantire la sicurezza dei lavoratori, anche quando lavorano da casa, per quanto ragionevolmente possibile. Le autorità locali hanno convenuto che sia Safe Work Australia (SWA), I'organo decisionale nazionale tripartito sulla salute e la sicurezza sul lavoro, la fonte principale di orientamento e raccomandazione sulla gestione dei rischi associati al COVID-19. L'ambito di competenza di SWA sulla gestione dei rischi associati al lavoro da casa comprende anche i rischi per la salute mentale derivanti dall'isolamento dai colleghi, dalle nuove incombenze lavorative e familiari, nonché i rischi di violenza familiare e domestica. ${ }^{2}$

- Inoltre, SWA ha fornito una guida per i datori di lavoro che offrono schemi di lavoro da casa, i quali dovrebbero tener conto della tipologia di lavoro e dell'idoneità dell'alloggio del dipendente. È stato sottolineato che possono essere necessarie soluzioni alternative per i lavoratori che subiscono violenza domestica o familiare. ${ }^{3}$

- L'Australian Skills Quality Authority, l'autorità nazionale australiana per l'istruzione e la formazione professionale (VET), ha messo a disposizione le risorse per i fornitori di VET per rimanere conformi alle norme quando si utilizza l'apprendimento a distanza. ${ }^{4}$

- II 9 aprile 2020, il governo federale australiano ha introdotto una serie di disposizioni temporanee nel Fair Work Act (Fair Work Act JobKeeper provisions), per sostenere l'attuazione e il funzionamento del sistema delle sovvenzioni salariali JobKeeper nei luoghi di lavoro. Una delle

\footnotetext{
${ }^{2}$ https://www.safeworkaustralia.gov.au/covid-19-information-workplaces/industry-information/general-industryinformation/working-home

${ }^{3}$ https://coronavirus.fairwork.gov.au/coronavirus-and-australian-workplace-laws/alternative-work-arrangementsduring-coronavirus
}

${ }^{4}$ https://www.asqa.gov.au/distance-learning 
disposizioni autorizza i datori di lavoro a dare istruzioni ai propri dipendenti affinché modifichino il luogo di lavoro, fatte salve alcune tutele. II luogo di lavoro può essere l'abitazione del dipendente. ${ }^{5}$

\section{Austria}

- II telelavoro è stato fortemente incoraggiato dalle autorità pubbliche durante la pandemia di COVID-19, e le norme ordinarie sono state applicate senza alcun cambiamento. II datore di lavoro non può prescrivere unilateralmente il telelavoro da casa. D'altro canto non vi è alcun diritto legale al telelavoro. II dipendente deve lavorare da casa solo previo accordo tra le parti. Gli accordi di telelavoro possono identificare il luogo di lavoro, la durata (limitata o illimitata), l'orario di lavoro giornaliero e settimanale, nonché i requisiti per l'assunzione dei costi per le spese private come elettricità, internet o hardware. ${ }^{6}$

- Una delle principali politiche adottate dal governo federale austriaco nel contesto della pandemia è stata il cosiddetto "Kurzarbeit", ovvero una riduzione dell'orario di lavoro finalizzata al mantenimento dei livelli occupazionali (una misura analoga è stata adottata in Germania). I dipendenti hanno diritto all' $80-90 \%$ del loro stipendio, mentre l'azienda copre solo il $10 \%$ dei costi salariali. A tale scopo il governo federale ha stanziato 4 miliardi di euro. ${ }^{7}$

- II Ministero Federale per lo Sviluppo Digitale ed Economico ha incoraggiato la costituzione del "Digital Team Austria", un gruppo di aziende informatiche che si impegnano ad offrire servizi digitali gratuiti alle PMI per almeno tre mesi al fine di facilitare il telelavoro. I servizi possono essere richiesti tramite i siti web dei rispettivi provider e comprendono soluzioni per videoconferenze e riunioni online, spazi di lavoro virtuali, sicurezza informatica, formazione digitale, ecc. ${ }^{8}$

\section{Belgio}

- Su richiesta del Ministro del Lavoro, le parti sociali del Consiglio Superiore per la Prevenzione e la Protezione sul Lavoro hanno redatto nel maggio 2020 una "Guida generica per combattere la diffusione del COVID-19 sul lavoro". II documento fornisce linee guida, raccomandazioni e consigli ai settori e datori di lavoro che hanno dovuto sospendere (parzialmente) le loro attività, al fine di preparare una ripresa in sicurezza del loro lavoro. Questa guida generica è personalizzabile a seconda dei diversi settori, per tener conto del contesto e delle esigenze specifiche. La consultazione tra datori di lavoro e dipendenti è stata fondamentale per garantire un'efficace attuazione. Le varie misure suggerite nella guida sono strettamente correlate tra loro e le misure tecniche e organizzative collettive per ridurre i rischi sono sempre preferite a quelle di protezione individuale. Le linee guida non sono vincolanti; la scelta circa la loro applicazione può variare da un'azienda all'altra, a seconda della situazione e delle circostanze specifiche. La guida si basa sui principi fondamentali dell'igiene e del distanziamento sociale e percorre una giornata di lavoro tipo per formulare una serie di punti cui prestare attenzione, misure e consigli riguardanti le diverse situazioni incontrate. ${ }^{9}$

\footnotetext{
${ }^{5} \mathrm{https}$ ://coronavirus.fairwork.gov.au/coronavirus-and-australian-workplace-laws/pay-and-leave-duringcoronavirus/jobkeeper-wage-subsidy-scheme

${ }^{6}$ https://news.wko.at/news/wien/So-klappt-die-Arbeit-von-zu-Hause-aus.html

${ }^{7}$ https://www.arbeiterkammer.at/beratung/arbeitundrecht/Arbeitszeit/SonderformenderArbeitszeit/Kurzarbeit.html

${ }^{8}$ https://www.usp.gv.at/Portal.Node/usp/public/content/home/532049.html

${ }^{9} \mathrm{https}$ ://www.vbo-feb.be/globalassets/publicaties/veilig-aan-het-werk---generieke-gids-om-de-verspreiding-van-covid19-op-het-werk-tegen-te-gaan/generieke-gids-v2-2020-05-04_fr---low.pdf
} 
- II Belgio ha concluso accordi reciproci con Francia, Germania, Lussemburgo e Paesi Bassi per evitare che i lavoratori frontalieri siano danneggiati dal COVID-19. A causa delle restrizioni di viaggio in vigore durante la pandemia, i lavoratori frontalieri corrono il rischio che il loro reddito da lavoro dipendente diventi pienamente imponibile nello Stato di residenza. Gli accordi di reciprocità con i quattro Paesi confinanti sopra menzionati mirano a garantire che i lavoratori frontalieri non subiscano svantaggi fiscali lavorando da casa. I giorni di lavoro da casa saranno assimilati ai giorni di lavoro nel Paese in cui il lavoratore avrebbe normalmente lavorato. Ciò significa che, nonostante il lavoro da casa, il reddito da lavoro può continuare ad essere tassabile nello Stato in cui si lavora. ${ }^{10}$

\section{Canada}

- Nel marzo 2020, i dipendenti del governo sono stati incoraggiati a lavorare da casa laddove possibile. La Canada School of Public Service ha pubblicato una lista di controllo per aiutare a prepararsi al lavoro a distanza, consigli per lavorare in modo efficace e guidare un team virtuale, nonché una guida su come creare un piano di lavoro per il team virtuale. ${ }^{11}$

- II Segretariato del Consiglio del Tesoro del Canada ha fornito una guida ai dipartimenti e alle agenzie con l'auspicio che i manager prendano in considerazione il telelavoro per tutti i loro dipendenti, in tutti i luoghi di lavoro, garantendo al contempo la continuità delle attività e dei servizi del governo. Questa direttiva fornisce il quadro di riferimento per la gestione del telelavoro per le persone dei dipartimenti essenziali dell'amministrazione pubblica. Elenca inoltre i ruoli e le responsabilità dei principali stakeholder e suggerisce un modello per la stesura di accordi di telelavoro completi. ${ }^{12}$

- Inoltre, alcuni dipartimenti federali, come il Public Services and Procurement Canada, hanno introdotto iniziative per garantire che i dipendenti abbiano gli strumenti necessari per lavorare in sicurezza da casa. Riconoscendo che il servizio pubblico federale continuerà a massimizzare il telelavoro nel prossimo futuro, i manager hanno ricevuto una guida per autorizzare la consegna delle sedie da ufficio dei dipendenti a domicilio o fornire loro un'indennità fino a 500 dollari canadesi per l'acquisto di attrezzature da ufficio da utilizzare a casa, come le scrivanie. ${ }^{13}$

- La chiusura di aziende non essenziali e le misure di salute pubblica messe in atto in tutto il Canada per rallentare la diffusione del COVID-19 hanno fatto sì che milioni di canadesi lavorassero da casa, venissero impiegati a orario ridotto o che diventassero disoccupati. Lo strumento online del servizio nazionale canadese per l'occupazione "Job Bank" ha modificato il proprio sito web e adattato i servizi per sostenere i lavoratori e i datori di lavoro durante la pandemia di COVID-19 e rispondere alle nuove realtà del mercato del lavoro introducendo una nuova funzione che permette ai candidati di cercare in modo specifico opportunità di telelavoro. ${ }^{14}$

- Anche prima della pandemia di COVID-19, il governo del Canada aveva sviluppato un meccanismo che permetteva ai lavoratori il cui "contratto di lavoro richieda di utilizzare una parte

\footnotetext{
${ }^{10} \mathrm{https}: / /$ home.kpmg/xx/en/home/insights/2020/05/flash-alert-2020-247.html

${ }^{11} \mathrm{https}: / / w w w . c s p s-e f p c . g c . c a / t o o l s / j o b a i d s /$ working-remotely-members-eng.aspx

${ }^{12} \mathrm{https}: / /$ www.tbs-sct.gc.ca/pol/doc-eng.aspx?id=32636

${ }^{13 h}$ https://www.tpsgc-pwgsc.gc.ca/comm/mc-cd/nouvelles-news/psm-fdm/2020-06-23-eng.html

${ }^{14}$ https://www.jobbank.gc.ca/findajob/resources/covid-19
} 
della loro abitazione per lavorare" di dedurre le spese legate all'attività lavorativa dal loro reddito imponibile. ${ }^{15}$

Cile

- Nel marzo 2020 il governo ha approvato una riforma del Codice del Lavoro per rafforzare le norme sul lavoro a distanza. Le nuove disposizioni consentono ai lavoratori e alle imprese di stipulare accordi sul telelavoro, chiarire gli obblighi dei datori di lavoro in materia di prevenzione dei rischi e di protezione dei lavoratori e introdurre il diritto alla disconnessione per i dipendenti. ${ }^{16}$

- Nel 2010 il Cile ha lanciato il programma "Start-up visa": si tratta di una politica nazionale per l'immigrazione volta ad attirare i fondatori di Start-up stranieri offrendo procedure amministrative snelle per la concessione di visti d'ingresso e permessi di soggiorno, nonché, occasionalmente, incentivi fiscali e sostegno finanziario. Nei suoi primi sette anni di attività, Start-Up Chile ha accolto più di 1600 startup (coinvolgendo 4500 imprenditori) da 85 Paesi, con una valutazione lorda del portafoglio di 1,4 miliardi di dollari e un miliardo di dollari di capitale raccolto. ${ }^{17}$

\section{Cina}

- Durante l'epidemia la Cina ha introdotto misure per promuovere l'adozione di nuove tecnologie, pratiche commerciali (ad esempio, vendita al dettaglio senza personale, consegne senza contatto, confezionamento standardizzato di alimenti freschi) e modelli commerciali (ad esempio, acquisti online, assistenza medica online, istruzione online, uffici e servizi online, intrattenimento digitale e vita digitale) da parte delle aziende. Per accelerare l'adozione delle tecnologie digitali la Cina supporta le PMI nell'accesso al cloud computing, al telelavoro, videoconferenze, formazione online, R\&S collaborativa e e-commerce. ${ }^{18}$

- Le imprese sono state incoraggiate a organizzare i tempi di inattività e le rotazioni dei propri dipendenti in modo da poter partecipare a vari corsi di formazione professionale online durante il periodo di sospensione o riduzione della produzione a causa della pandemia. Esse riceveranno sovvenzioni per i costi di formazione. II 12 febbraio 2020 il Ministero delle Risorse Umane e della Previdenza Sociale ha lanciato una piattaforma di formazione online gratuita per sostenere lo sviluppo dell'istruzione e della formazione professionale online. Anche le PMI sono state incoraggiate a partecipare alla formazione online per affinare le loro competenze durante l'epidemia da nuovo coronavirus. Attraverso piattaforme di telelavoro mobile sono stati offerti gratuitamente alle PMI una serie di corsi sull'interpretazione delle politiche e di know-how tecnico e gestionale. ${ }^{19}$

- China Telecom ha fornito servizi di ufficio gratuiti su cloud per le PMI gravemente colpite dalla pandemia. Allo stesso tempo, ha offerto tre mesi di servizi cloud gratuiti alle PMI di tutto il Paese. ${ }^{20}$

\footnotetext{
${ }^{15} \mathrm{https}$ ://www.canada.ca/en/revenue-agency/services/forms-publications/publications/t4044/employmentexpenses.html

${ }^{16} \mathrm{https}: / /$ www.leychile.cl/Navegar?idNorma $=1143741$

${ }^{17}$ https://www.startupchile.org/impact/

18 https://www.urdupoint.com/en/technology/china-to-empower-smes-with-digital-technolog-868855.html; http://www.g20e.tsinghua.edu.cn/EN/contents/1185/1308.html

${ }^{19} \mathrm{http}: / / w w w . g 20$ e.tsinghua.edu.cn/EN/contents/1185/1304.html

${ }^{20}$ http://www.g20e.tsinghua.edu.cn/EN/contents/1185/1308.html
} 


\section{Colombia}

- Il 17 marzo 2020 il Ministero del Lavoro ha emanato la circolare n. 21 che specifica le norme e le soluzioni per lo sviluppo ottimale del lavoro da casa, il telelavoro, l'orario di lavoro flessibile, le ferie anticipate o collettive, e altro. ${ }^{21}$

\section{Corea del Sud}

- Temporanea semplificazione delle procedure per i datori di lavoro che richiedono una sovvenzione (fino a 2 milioni di won all'anno per dipendente) per l'introduzione di modalità di lavoro flessibili come il lavoro da casa, il lavoro a distanza o l'orario di lavoro selettivo. ${ }^{22}$

- La Corea ha introdotto misure per incoraggiare i negozi tradizionali ad aprire una propria attività online. ${ }^{23}$

\section{Estonia}

- E-Residency è un programma governativo lanciato alla fine del 2014. Permette ai non estoni di accedere a servizi pubblici locali quali la costituzione di società, il sistema bancario, l'elaborazione dei pagamenti e la tassazione. I principali vantaggi derivanti dalla residenza digitale sono l'indipendenza dalla localizzazione, che consente la gestione a distanza, e l'accesso al mercato unico dell'Unione Europea. ${ }^{24}$

\section{Francia}

- Nel maggio 2020 il Ministero del Lavoro francese ha pubblicato delle FAQ sul telelavoro, redatte in consultazione con le parti sociali, per aiutare le imprese e i dipendenti che non avevano ancora adottato questa modalità a causa dell'assenza di un accordo in vigore o di difficoltà a trovare una soluzione adeguata. ${ }^{25}$

- Sono stati conclusi accordi di reciprocità con la Germania, il Belgio e la Svizzera per stabilire regimi fiscali specifici per i lavoratori frontalieri. ${ }^{26}$

\section{Germania}

- Nell'ambito del preesistente programma "go-digital", il governo federale ha istituito un nuovo programma di finanziamento rivolto alle $\mathrm{PMI}$ e alle imprese artigiane per incentivare la creazione di posti di lavoro da casa. II sostegno finanziario consiste nel rimborso fino al $50 \%$ delle spese sostenute per servizi forniti da una società di consulenza autorizzata dal Ministero dell'Economia. I servizi comprendono l'allestimento di soluzioni di e-commerce, home office e cyber security, ad esempio attraverso la configurazione di software e hardware. II massimale per le tariffe giornaliere di consulenza ammonta a 1100 euro, per un massimo di 30 giorni. Le PMI e le imprese artigiane

\footnotetext{
${ }^{21} \mathrm{https}: / / w w w . m i n e d u c a c i o n . g o v . c o / 1759 /$ w3-article-394115.html?_noredirect=1

${ }^{22}$ http://moel.go.kr/english/poli/poliNewsnews_view.jsp?idx=1552

${ }^{23} \mathrm{https}: / /$ home.kpmg/xx/en/home/insights/2020/04/south-korea-government-and-institution-measures-in-response-tocovid.html

${ }^{24}$ https://e-resident.gov.ee/become-an-e-resident/

${ }^{25} \mathrm{https}$ ://ravail-emploi.gouv.fr/IMG/pdf/qr-teletravail-deconfinement.pdf

${ }^{26} \mathrm{https}$ ://de.ambafrance.org/COVID-19-situation-des-travailleurs-frontaliers
} 
interessate al programma hanno a disposizione una mappa dedicata per trovare un consulente nella loro regione.

- La Germania ha concluso accordi di reciprocità con Austria, Lussemburgo e Paesi Bassi sulla tassazione dei lavoratori frontalieri alla luce delle circostanze derivanti dalla pandemia di COVID19. I lavoratori che vivono al di fuori della Germania ma che vi lavorano sono normalmente soggetti in Germania a una tassazione sul loro reddito da lavoro come non residenti fiscali. A tali lavoratori frontalieri spettano quindi specifici sgravi fiscali. Uno dei criteri principali per poterne beneficiare è che almeno il $90 \%$ del reddito totale sia soggetto all'imposta tedesca sul reddito (reddito maturato in Germania) nell'arco dell'anno solare. Poiché il numero di giorni lavorativi in Germania si è ridotto a causa del COVID-19, il reddito di origine tedesca potrebbe non raggiungere più la soglia del 90\% del reddito totale per l'anno civile 2020. Gli accordi di reciprocità firmati dalla Germania con Austria, Lussemburgo e Paesi Bassi stabiliscono che il reddito da lavoro dipendente relativo ai giorni lavorativi trascorsi a distanza a causa delle misure di prevenzione dal COVID-19 può rimanere tassabile nello Stato in cui si trova il luogo di lavoro abituale. ${ }^{27}$

\section{Grecia}

- Durante la pandemia le imprese sono state incoraggiate a facilitare gli accordi di telelavoro fino a nuovo avviso, nel caso in cui l'attività non fosse stata sospesa. Per i dipendenti che lavorano nel settore privato, il datore di lavoro può imporre il telelavoro. Tale accordo doveva essere comunicato elettronicamente al sistema informatico ERGANI del Ministero del Lavoro e degli Affari Sociali (MoLSA) per ogni lavoratore coinvolto. ${ }^{28}$

- L'Autorità Ellenica per la Protezione dei Dati (HDPA) ha pubblicato, il 15 aprile 2020, le linee guida sul telelavoro alla luce della crisi del COVID-19 per garantire la sicurezza e il rispetto del regolamento generale sulla protezione dei dati (regolamento (UE) 2016/679). In particolare, le linee guida prendono atto dell'obbligo del datore di lavoro di formare i dipendenti al telelavoro e della maggiore aspettativa di privacy per i dipendenti che lavorano da casa, delineando al contempo le regole sull'accesso a internet, sull'uso della posta elettronica, dei dispositivi e delle teleconferenze. Inoltre le linee guida suggeriscono l'uso della crittografia e dei firewall, la divisione tra dati relativi al lavoro e dati personali e l'uso di un solido sistema WAP2 quando i dipendenti utilizzano il Wi-Fi. ${ }^{29}$

\section{Irlanda}

- Enterprise Ireland, l'organizzazione governativa irlandese responsabile dello sviluppo e della crescita delle imprese irlandesi nei mercati mondiali, ha creato una pagina web dedicata al COVID19 poco dopo lo scoppio della pandemia. La pagina web fornisce informazioni pratiche su una serie di argomenti, tra cui il lavoro a distanza, l'accesso a strumenti digitali e strategie per la gestione di team virtuali. ${ }^{30}$

\footnotetext{
${ }^{27}$ https://home.kpmg/xx/en/home/insights/2020/04/flash-alert-2020-191.html

${ }^{28} \mathrm{https}$ ://www.ilo.org/global/topics/coronavirus/country-responses/lang--en/index.htm\#GR

${ }^{29} \mathrm{https}$ //www.dataguidance.com/news/greece-hdpa-publishes-teleworking-guidelines

${ }^{30}$ https://eilearn.ie/covid-19/
} 
Italia

- Sono stati introdotti accordi di lavoro con modalità semplificate sul telelavoro per tutte le attività e le professioni per le quali è possibile, procedure semplificate per la richiesta e la notifica del telelavoro nonché procedure di gara semplificate per il settore pubblico. ${ }^{31}$

- II Ministero per I'Innovazione Tecnologica e la Digitalizzazione ha lanciato l'iniziativa "Solidarietà digitale". Questa include un portale dove le aziende (in particolare le PMI e i lavoratori autonomi) possono registrarsi per accedere ai servizi digitali messi a disposizione gratuitamente per un periodo limitato dalle grandi aziende del settore privato in ambiti quali il telelavoro, le conferenze online, l'accesso ai dati mobili, il cloud computing, ecc. ${ }^{32}$

\section{Giappone}

- Già prima della pandemia il governo giapponese aveva adottato una serie di misure, come la concessione di sovvenzioni per l'adozione del telelavoro, l'emanazione di linee guida e il miglioramento dei sistemi di consulenza.

- Nel marzo 2020, il governo giapponese ha introdotto una sovvenzione specifica per le PMI che adottano il telelavoro come risposta alla pandemia. II regime copre il 50\% (fino a 1 milione di yen: circa 8100 euro) dei costi per l'adozione di soluzioni informatiche, la creazione o la modifica dei regolamenti di lavoro e la formazione dei lavoratori, ecc. ${ }^{33}$

\section{Malesia}

- La Malaysia Digital Economy Corporation, creata dal governo come parte della strategia digitale del Paese, offre un ampio elenco di soluzioni digitali per le PMI da parte delle aziende tecnologiche malesi. $^{34}$

\section{Messico}

- II Ministero del Lavoro e della Previdenza Sociale ha pubblicato una guida per orientare le imprese a fronte della crisi del COVID-19, affinché possano adottare misure per la salute e sicurezza sul lavoro e promuovere modalità di lavoro a distanza, come il telelavoro.

- Inoltre, è stato lanciato il micro-sito "Insieme per il lavoro" che contiene la guida sopramenzionata, una sezione sulla formazione a distanza, il patrocinio e la consulenza legale e il sostegno ai consumi locali. ${ }^{35}$

\footnotetext{
${ }^{31} \mathrm{https://www.lavoro.gov.it/strumenti-e-servizi/smart-working/Pagine/default.aspx}$

${ }^{32} \mathrm{https://solidarietadigitale.agid.gov.it/}$

${ }^{33}$ https://alesia-law.com/en/2020/04/grants-and-subsidies-for-smes-in-japan-under-covid-19-situation/

${ }^{34} \mathrm{https}: / / m d e c . m y / w p-c o n t e n t /$ uploads/SME-Digital-Quickwins-.pdf

${ }^{35}$ https://juntosporeltrabajo.stps.gob.mx/
} 


\section{Nuova Zelanda}

- Il governo ha incentivato i datori di lavoro a trattenere i propri dipendenti attraverso un programma di sussidi salariali e ha incoraggiato le aziende a sfruttare le opzioni di lavoro da casa. Solo le aziende che soddisfano i requisiti di attività essenziale sono state autorizzate a rimanere aperte. ${ }^{36}$

\section{Polonia}

- Anche se il Codice del Lavoro polacco non disciplina il telelavoro, i datori di lavoro hanno potuto adottarlo per un certo periodo di tempo, per motivi di sicurezza e di salute. ${ }^{37}$

\section{Portogallo}

- Durante lo stato di emergenza il telelavoro era considerato obbligatorio per i lavori compatibili con il lavoro da casa. ${ }^{38}$ I lavoratori potevano decidere unilateralmente di iniziare il telelavoro purché la loro funzione fosse con esso compatibile. A partire dal 16 marzo 2020 è stata offerta questa opportunità anche ai dipendenti pubblici. ${ }^{39}$

- Il governo, in collaborazione con le aziende del comparto tecnologico, ha offerto una serie di strumenti digitali gratuiti in portoghese a sostegno del telelavoro, con un centro di contatto per fornire assistenza. ${ }^{40}$

\section{Slovacchia}

- II 31 marzo 2020 il governo della Repubblica Slovacca ha approvato importanti modifiche al Codice del Lavoro e ai relativi regolamenti, tra cui: l'introduzione della possibilità di imporre al lavoratore di lavorare da casa e del diritto dello stesso di accedere al telelavoro; la riduzione dell'indennità salariale all' $80 \%$ nel caso sussistano difficoltà da parte del datore di lavoro durante e a causa del lockdown, e la possibilità per il datore di lavoro di ordinare al lavoratore di lavorare al di fuori del tempo per il quale è stata pagata l'indennità salariale; la riduzione ad almeno due giorni del periodo di preavviso per comunicare l'orario di lavoro da parte del datore di lavoro e la riduzione del preavviso per comunicare il periodo di ferie (imposte); una maggiore protezione dei lavoratori rispetto alla cessazione del rapporto di lavoro e alla reintroduzione al lavoro per motivi legati al COVID-19. 41

\section{Slovenia}

- Aiuti alle aziende nella gestione del telelavoro e di casi di quarantena. ${ }^{42}$

\footnotetext{
${ }^{36} \mathrm{https://ssc.govt.nz/resources/covid-19-state-services-workforce-guidelines/}$

${ }^{37}$ https://www.roedl.com/insights/covid-19/poland-home-office-employer-employee-corona-virus

${ }^{38} \mathrm{https}: / /$ dre.pt/web/guest/home/-/dre/131068124/details/maximized

${ }^{39} \mathrm{https}: / /$ dre.pt/home/-/dre/130243053/details/maximized

${ }^{40}$ https://covid19estamoson.gov.pt/teletrabalho/

${ }^{41} \mathrm{https}$ ///www.whitecase.com/publications/alert/overview-most-recent-measures-government-slovak-republicregarding-spread-covid

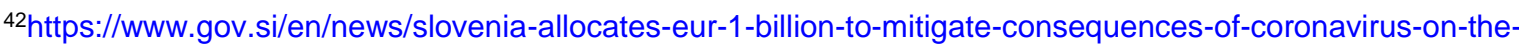
economy/
} 
Spagna

- Durante la crisi di COVID-19 il telelavoro è stato fortemente incoraggiato al fine di evitare la riduzione dell'attività economica e i licenziamenti. ${ }^{43}$

- II programma Acelera PYME (250 milioni di euro) offre misure per la digitalizzazione delle PMI attraverso sovvenzioni e prestiti, per finanziare investimenti in attrezzature digitali o soluzioni per il lavoro a distanza. ${ }^{4}$

\section{Svizzera}

- Ove possibile si incoraggiano orari flessibili e il telelavoro. ${ }^{45}$

\section{Turchia}

- L'Agenzia Turca per il Lavoro (IŞKUR) intende aumentare l'occupazione e l'occupabilità dei giovani attraverso programmi attivi del mercato del lavoro come la formazione sul posto di lavoro, la formazione e l'orientamento professionale. Il suo progetto Job Clubs Project, in particolare, è un programma intensivo di orientamento e formazione professionale che fornisce supporto metodologico e motivazionale a specifici gruppi target come donne, giovani, disabili, disoccupati di lungo periodo e altri. I programmi di formazione dei Job Clubs sono attivi in tutto il Paese e durano dai 2 ai 5 giorni. I partecipanti si uniscono a gruppi di 6-15 persone e ricevono consigli su come preparare un curriculum vitae efficace, nonché informazioni sui canali per la ricerca di occupazione e sul mercato del lavoro. Dopo lo scoppio della pandemia, i Job Clubs e altri servizi come l'orientamento agli studenti e ai datori di lavoro hanno iniziato a essere offerti online, coinvolgendo chi cerca lavoro e chi lo offre attraverso i social media e altri canali online ${ }^{46}$

\section{Stati Uniti d'America}

- Nell'aprile 2020 i Centri per la prevenzione e il controllo delle malattie (Centers for Disease Control and Prevention - CDC) hanno pubblicato una guida aggiornata che fornisce indicazioni alla popolazione circa la necessità di indossare le mascherine e seguire linee guida di sicurezza aggiornate per i lavoratori che operano in situazioni critiche. I CDC hanno mantenuto un ruolo di guida per le aziende e i datori di lavoro nell'elaborazione di piani e risposte alle situazioni legate al COVID-19. Tra queste vanno incluse le strategie per rendere flessibili i luoghi di lavoro (ad esempio il telelavoro), orari di lavoro flessibili (turni scaglionati) e per aumentare lo spazio fisico tra i dipendenti sul posto di lavoro. ${ }^{47}$

- Con la maggior parte della nazione che si trova ad avere a che fare con limitazioni sui viaggi d'affari e obblighi di rimanere a casa, diversi stati e località hanno pubblicato una guida sull'impatto che i disagi provocati dal COVID-19 avranno sugli obblighi di ritenuta fiscale per i datori di lavoro multi-statali. Gli Stati hanno una vasta gamma di risposte:

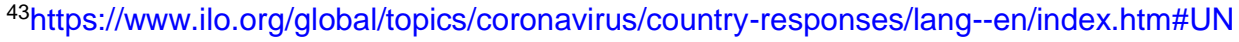

${ }^{44}$ https://acelerapyme.gob.es/soluciones-tecnologicas/teletrabajo

${ }^{45} \mathrm{https}$ ://www.ilo.org/global/topics/coronavirus/country-responses/lang--en/index.htm\#UN

46 https://ec.europa.eu/transparency/regdoc/rep/10102/2020/EN/SWD-2020-68-F1-EN-MAIN-PART-1.PDF

${ }^{47}$ https://www.cdc.gov/coronavirus/2019-ncov/community/guidance-business-response.html
} 
a. II New Jersey e il Mississippi hanno modificato le loro politiche fiscali per consentire alle aziende di mantenere la stessa ritenuta per la sede temporanea di telelavoro dei dipendenti durante la pandemia.

b. Minnesota e Maryland hanno pubblicato una guida che indica che i telelavoratori possono creare nuovi obblighi di ritenuta.

c. L'Ohio ha approvato una legge che stabilisce che il lavoro a distanza dovuto alla pandemia non conta ai fini della soglia di trattenuta per le imposte comunali sul reddito.

d. Ai fini dell'imposta sul reddito delle società, il Distretto di Columbia, Indiana e Nord Dakota hanno indicato che non imporranno il nesso di imposta sul reddito delle società a causa della presenza temporanea di nuovi telelavoratori, senza specificare l'impatto che ciò avrà sugli obblighi di ritenuta alla fonte..$^{48}$

\section{Ungheria}

- Modifiche temporanee alla normativa sul lavoro per consentire il lavoro a distanza, da casa e gli orari di lavoro flessibili, autorizzando inoltre i datori di lavoro ad adottare le misure necessarie per controllare lo stato di salute dei dipendenti. ${ }^{49}$

\section{Politiche regionali e locali per il telelavoro}

Abruzzo (Italia)

- Nell'aprile del 2020 l'Abruzzo ha adottato il quadro di riferimento "Linee guida per l'utilizzo della formazione a distanza/e-learning" definito nel 2019 dalla Conferenza delle Regioni e delle Province Autonome, che consente l'apprendimento a distanza nelle attività di formazione professionale gestite dagli enti regionali. 50

\section{Barcellona (Spagna)}

- Barcelona Activa, l'agenzia municipale di sostegno alle imprese, gestisce una rete di centri informativi che forniscono assistenza on-line e telefonica in materia di lavoro nell'ambito della pandemia di COVID-19, ad esempio riguardo ai licenziamenti temporanei, al telelavoro, alla prevenzione dei rischi e all'igiene sul posto di lavoro. II 9 aprile 2020, il centro di formazione e diffusione tecnologica di Barcelona Activa, denominato Cibernàrium, ha ricevuto più di 5800 domande di iscrizione, il triplo rispetto all'ordinario. I temi che riscuotono il maggiore interesse da parte degli utenti riguardano l'efficacia degli incontri virtuali, l'uso delle piattaforme collaborative e le modalità di telelavoro. ${ }^{51}$

\footnotetext{
${ }^{48} \mathrm{https}: / / w w w . j d s u p r a . c o m / l e g a l n e w s / c o v i d-19-t e l e w o r k-t r i g g e r s-s t a t e-t a x-61603 /$

49 https://www.ilo.org/global/topics/coronavirus/country-responses/lang--en/index.htm\#UN; http://www.cielolaboral.com/wp-content/uploads/2020/04/hungria_noticias_cielo_coronavirus.pdf

50http://www2.consiglio.regione.abruzzo.it/leggi_tv/abruzzo_Ir/2020/Ir20009/Intero.asp

51 https://cibernarium.barcelonactiva.cat/
} 
Basilicata (Italia)

- Nel marzo 2020 la Basilicata ha istituito un regime di sostegno alle imprese che adottano piani di telelavoro: le PMI, i lavoratori autonomi e i liberi professionisti possono ricevere aiuti fino al $70 \%$ della spesa sostenuta per l'attivazione di piani di telelavoro per i propri dipendenti. II budget complessivo è di 3 milioni di euro, che in parte attinge al Fondo Europeo di Sviluppo Regionale (FESR). Gli investimenti ammissibili comprendono beni strumentali materiali e immateriali per un contributo massimo di 200000 di euro per azienda. ${ }^{52}$

\section{Braga (Portogallo)}

- L'agenzia municipale per lo sviluppo economico InvestBraga e la sua filiale per l'innovazione Startup Braga hanno organizzato una serie di corsi online e webinar per aiutare le PMI a rafforzare le loro competenze digitali in settori quali l'e-commerce, il telelavoro e le videoconferenze. L'agenzia offre anche consulenza gratuita su diverse tematiche digitali. ${ }^{53}$

\section{Bristol (Regno Unito)}

- II progetto Caboodle Collaborative Workspace a Bristol, Regno Unito, si rivolge agli imprenditori home-based che necessitano di sostegno nell'assistenza all'infanzia. Lanciato nel 2017, offre sessioni "pop-up" di co-working con un asilo nido in loco, consentendo a genitori e agli educatori di lavorare fianco a fianco mentre i bambini sono accuditi nelle vicinanze. ${ }^{54}$

\section{Friuli-Venezia Giulia (Italia)}

- II 5 maggio 2020 questa Regione autonoma italiana ha pubblicato un bando per la concessione di contributi a lavoratori autonomi, cooperative e piccole imprese con un massimo di 50 dipendenti per supportare l'adozione di piani di telelavoro. II budget complessivo del programma è di 2,4 milioni di euro, tratti dal Fondo Sociale Europeo (FSE). Le sovvenzioni coprono tutti i costi sostenuti durante l'intero processo di transizione al telelavoro, dall'analisi esplorativa e la consulenza alla formazione, fino all'acquisto di software e hardware (sovvenzioni fino a 11700 euro per ogni singola impresa). ${ }^{55}$

- Sostegno agli enti pubblici incaricati della formazione professionale per rafforzare la loro capacità di erogare formazione a distanza. A tal fine è stato stanziato un budget complessivo di 0,5 milioni di euro, attingendo al FSE. ${ }^{56}$

\section{Île-de-France (Francia)}

- La Regione dell'Île-de-France fornisce servizi di supporto gratuiti per garantire che gli enti qualificati possano continuare le loro attività di formazione durante la pandemia di COVID-19. Per fare ciò, la Regione ha collaborato con Ecole O'clock, un fornitore di formazione a distanza. Gli enti di formazione sono tenuti a compilare un modulo online che definisce le loro specifiche

\footnotetext{
${ }^{52}$ https://portalebandi.regione. basilicata.it/PortaleBandi/detail-bando.jsp?id=581951

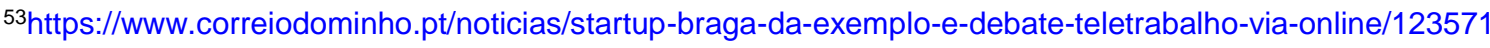

${ }^{54} \mathrm{http}: / /$ workandhome.ac.uk/2017/11/07/caboodle-co-working-with-childcare

55 http://www.regione.fvg.it/rafvg/cms/RAFVG/formazione-lavoro/lavoro/FOGLIA81/articolo.html\#id1

${ }^{56}$ http://mtom.regione.fvg.it/storage//2020_517/Allegato\%201\%20alla\%20Delibera\%20517-2020.pdf
} 
esigenze di formazione per poter beneficiare del programma di supporto. Alle classi virtuali possono partecipare da 20 a 50 tirocinanti. $^{57}$

\section{Lazio (Italia)}

- Nel marzo 2020 la Regione ha pubblicato un bando per incentivi a lavoratori autonomi e PMI che adottano piani di telelavoro. I contributi vanno da 7500 a 22500 euro, a seconda delle dimensioni dell'impresa. I costi ammissibili al rimborso comprendono quelli sostenuti per la consulenza, la formazione e la tecnologia digitale. A tal fine è stato stanziato un totale di 2 milioni di euro, attingendo al FSE. ${ }^{58}$

\section{Liguria (Italia)}

- La Regione ha stanziato 600000 euro, attingendo dal FSE, per i voucher alle PMI che adottano piani di telelavoro. I benefici coprono il $60 \%$ degli investimenti per la formazione e le tecnologie digitali. Gli investimenti ammissibili possono variare da 1000 a 5000 euro. ${ }^{59}$

- I disoccupati temporanei possono beneficiare di un percorso integrato di politica attiva del lavoro che combina l'apprendimento a distanza (o, in alternativa, la formazione intelligente) con un sostegno al reddito sotto forma di una sovvenzione mensile di 500 euro per un massimo di cinque mesi. I beneficiari possono anche richiedere un voucher per l'acquisto o il noleggio di tecnologie digitali. II programma, denominato "Smart@ttivo", ha un budget complessivo di 3,6 milioni di euro (FSE). ${ }^{60}$

\section{Lombardia (Italia)}

- Nel marzo 2020 la Regione ha istituito un programma pubblico per sostenere l'adozione di piani di telelavoro. Sovvenzioni fino a 15000 euro copriranno i costi sostenuti per la formazione digitale e l'acquisto di tecnologie informatiche (4,5 milioni di euro stanziati). ${ }^{61}$

\section{Milano (Italia)}

- Nell'aprile 2020 il Comune ha pubblicato "Milano 2020. Strategia di adattamento", aperta alla consultazione pubblica. II documento sottolinea la necessità di mantenere il telelavoro come scenario privilegiato nei prossimi mesi. ${ }^{62} \mathrm{Nel}$ maggio 2020 l'amministrazione comunale ha

\footnotetext{
57https://www.defi-metiers.fr/dossiers/covid-19-continuite-daction-pour-lemploi-et-la-formation

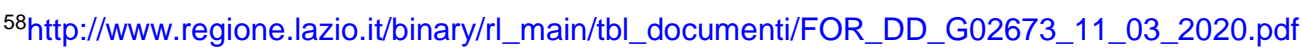
http://www.regione.lazio.it/binary/rl_main/tbl_documenti/FOR_DD_G02673_11_03_2020_Allegato_1.pdf

${ }^{59} \mathrm{https}$ //www.regione.liguria.it/homepage/salute-e-sociale/101395-coronavirus/25311-bando-dotazione-eformazione-smart-working.html

${ }^{60}$ https://www.regione.liguria.it/eventi-live/coronavirus-17-aprile-2020

${ }^{61} \mathrm{https}$ ://www.lombardianotizie.online/coronavirus-smartworking/

${ }^{62} \mathrm{https}: / /$ www.comune.milano.it/documents/20126/95930101/Milano+2020.++Strategia+di+adattamento.pdf/c96c129 7-f8ad-5482-859c-90de1d2b76cb?t=1587723749501
} 
concordato con i principali datori di lavoro locali che l'80\% della forza lavoro dovrà continuare a lavorare da casa. ${ }^{63}$

- La Città, in collaborazione con la locale camera di commercio e i sindacati, ha inoltre attuato un programma chiamato "Partita AttIVA" per sostenere i lavoratori autonomi e i liberi professionisti che operano in gran parte da casa - nello sviluppo del loro piano aziendale, attraverso l'erogazione di prestiti a tasso d'interesse ridotto fino a 15000 euro e servizi di tutoraggio. Sono incoraggiati gli investimenti nella formazione e nelle attrezzature digitali. ${ }^{64}$

\section{Molise (Italia)}

- II bando pubblico "lo lavoro agile" è stato lanciato nel marzo 2020 per promuovere il telelavoro tra le PMI. I contributi individuali possono andare da 10000 a 50000 euro. II budget totale è di 0,46 milioni di euro (FESR-FSE). ${ }^{65}$

\section{Normandia (Francia)}

- A partire dalla fine di marzo 2020 la Regione della Normandia ha fornito una serie di servizi a sostegno dell'apprendimento e della formazione a distanza. Questi includono la creazione di forum online per gli enti di formazione per porre domande e ricevere un supporto mirato alla formazione a distanza, e una serie di dieci webinar per guidare le organizzazioni di formazione nell'offerta di formazione a distanza. ${ }^{66}$

\section{Occitania (Francia)}

- La Regione ha sviluppato un piano che mira a "formare invece di licenziare" (former plutôt que licencier), che mette a disposizione 4 milioni di euro a sostegno delle persone in cerca di lavoro che desiderano continuare o intraprendere percorsi di formazione a distanza. Nell'ambito del piano, la Regione ha ampliato il budget a disposizione dei cosiddetti "Opérateurs de Compétences", il cui ruolo è finanziare la formazione e accompagnare le PMI nella definizione dei bisogni formativi. II piano si basa sulla vasta esperienza della Regione nel sostenere la formazione a distanza attraverso la sua piattaforma di formazione online. ${ }^{67}$

\section{Paesi Baschi (Spagna)}

- L'agenzia regionale per lo sviluppo economico, denominata Grupo SPRI, ha lanciato due iniziative per favorire il telelavoro tra le PMI nell'ambito della pandemia di COVID-19:

- Inplantalariak: un team di consulenti tecnologici fornisce supporto gratuito a lavoratori autonomi e alle microimprese con meno di dieci dipendenti nella ricerca di soluzioni per il telelavoro, il monitoraggio e la gestione a distanza, la sicurezza informatica e il commercio elettronico;

\footnotetext{
${ }^{63} \mathrm{https}: / /$ www.ansa.it/lombardia/notizie/2020/05/03/comune-di-milano-alle-aziende-Stati Unitite-lo-smartworking_d316a538-1776-4c77-b0ca-cf93d41dbf79.html

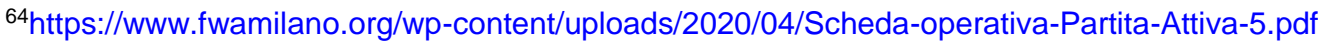

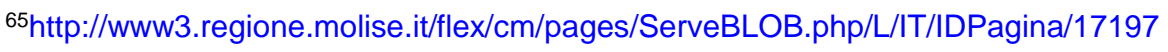

${ }^{66} \mathrm{https}: / /$ www.profildinfo.fr/index.php?|v|=notice_display\&id=55834\&opac_view=6\&opac_view=6

${ }^{67}$ https://www.laregion.fr/La-formation-au-secours-de-I-emploi?fbclid=IwAR3VpqZpUQM1jeX-

7V8dx15uQM49_a1XHRdaXmdumntkgoJh2eOIP2PJtmo
} 
Industria Digitala: supporta l'implementazione di soluzioni informatiche nelle PMI industriali, sovvenzionando tra il $25 \%$ e il $50 \%$ (a seconda della dimensione dell'impresa) delle spese sostenute per la consulenza digitale, l'ingegneria e l'acquisizione di hardware e software per promuovere il telelavoro. Le aziende possono beneficiare di un ulteriore $5 \%$ se sono in possesso di un certificato di qualità Bikain per l'uso della lingua basca o di un piano di assunzioni bilanciato rispetto al genere e promuovono l'uso di software in lingua basca. Lo stanziamento totale per il programma è di 2,3 milioni di euro, in parte a carico del FESR. ${ }^{68}$

\section{Pechino (Cina)}

- Nel febbraio 2020 il governo municipale ha annunciato un pacchetto di politiche per le PMI che comprendono materie quali tassazione, finanza, previdenza sociale, sovvenzioni e riduzione degli affitti. In particolare, le PMI potranno beneficiare di sovvenzioni per la formazione dei dipendenti e per gli investimenti legati al telelavoro. ${ }^{69}$

- L'associazione Beijing Business Incubation Association ha offerto, gratuitamente o a prezzi ridotti, spazi di co-working a micro e piccole imprese durante la pandemia di coronavirus. Gli 86 incubatori presenti in tutta la città possono ospitare circa 6000 piccole imprese. Secondo le stime, queste risparmieranno 43 milioni di dollari per l'affitto di uffici. ${ }^{70}$

\section{Piemonte (Italia)}

- Nel marzo 2020 la Regione ha ampliato un programma per il telelavoro precedente al COVID-19, aumentandone il budget a 4,5 milioni di euro (FSE). Sono incoraggiate le richieste congiunte di diverse PMI, con l'obiettivo di finanziare servizi condivisi (ad esempio, l'assistenza all'infanzia) per migliorare l'equilibrio tra lavoro e vita privata dei lavoratori e in particolare dei genitori. ${ }^{71}$

\section{Puglia (Italia)}

- Nel marzo 2020 la Puglia ha pubblicato un bando per sostenere l'attivazione di "Piani di innovazione Family Friendly" da parte delle PMI. I piani ammissibili alle sovvenzioni dovrebbero mirare alla destandardizzazione degli orari di lavoro (ad esempio attraverso un maggiore utilizzo di orari flessibili o del time banking) o all'istituzione di nuove modalità di lavoro, come il telelavoro. Lo stanziamento finanziario per questo programma ammonta a 14,5 milioni di euro provenienti dal FSE. II contributo massimo per ogni progetto è di 100000 euro. I contributi possono coprire fino all' $80 \%$ delle spese sostenute per la stesura e l'attuazione del piano e fino al $100 \%$ dei relativi costi di formazione. ${ }^{72}$

\footnotetext{
${ }^{68} \mathrm{https} / / / w w w . s p r i . e u s / e s / a y u d a s /$

${ }^{69} \mathrm{https}$ ://www.china-briefing.com/news/chinas-latest-regional-measures-to-support-smes-during-coronavirusoutbreak/?utm_source=Mondaq\&utm_medium=syndication\&utm_campaign=Linkedln-integration

70http://www.ebeijing.gov.cn/Home/News/t1622522.htm

${ }^{71} \mathrm{https}$ ://www.regione.piemonte.it/web/pinforma/notizie/oltre-45-milioni-per-lavoro-agile-nelle-aziende-private

72 https://www.regione.puglia.it/web/welfare-diritti-e-cittadinanza/-/attivazione-di-un-piano-di-innovazione-familyfriendly-nelle-pmi-po-puglia-2014-2020-fse-asse-viii-azione-8.6-b
} 


\section{Québec (Canada)}

- Nel contesto della pandemia di COVID-19, la locale agenzia delle entrate non considera come un beneficio imponibile il rimborso fino a 500 dollari canadesi per le attrezzature informatiche 0 d'ufficio che un dipendente ha acquistato per il telelavoro. ${ }^{73}$

- A causa dell'impatto economico della pandemia di COVID-19, il governo provinciale del Québec ha creato il "Panier Bleu", una piattaforma gestita dal governo che promuove i negozi locali che vendono online. La piattaforma in sé non supporta le transazioni, ma serve come risorsa per gli utenti che cercano aziende che vendono prodotti online durante la pandemia. ${ }^{74}$

\section{Contea di Santa Clara (Stati Uniti)}

- Nel maggio 2020 il Consiglio dei Supervisori della Contea di Santa Clara ha approvato una raccomandazione per continuare a promuovere il telelavoro dopo la fine della crisi di COVID-19, riconoscendo il miglioramento della qualità dell'aria e la riduzione del traffico nella Bay Area da quando sono entrate in vigore le politiche di contenimento della pandemia e molti lavoratori dipendenti hanno iniziato a lavorare da casa. L'obiettivo a lungo termine è quello di mantenere alti livelli di produttività riducendo al contempo l'impatto sul clima. La raccomandazione cerca anche di coinvolgere i datori di lavoro privati nella riduzione delle emissioni di gas serra proponendo una "Clear Air Telecommute Challenge" per incoraggiare i grandi gruppi ad aumentare il numero di dipendenti che telelavora. ${ }^{75}$ II Silicon Valley Leadership Group, un'organizzazione di politica pubblica che rappresenta più di 350 aziende, sostiene l'idea e la partecipazione del settore imprenditoriale nella formulazione del piano. ${ }^{76}$

\section{Schleswig-Holstein (Germania)}

- II progetto pilota CoWordLand lanciato dallo Schleswig-Holstein nel 2019 mirava a realizzare in via sperimentale spazi di co-working nelle aree rurali come nuova soluzione per riqualificare gli edifici vuoti e combattere l'esodo dei giovani. ${ }^{77}$

\section{Shanghai (Cina)}

- L'Ufficio Comunale delle Risorse Umane e Previdenza Sociale ha introdotto delle sovvenzioni per spese legate alla formazione. La misura prevede che coloro che organizzano la partecipazione dei propri dipendenti a vari percorsi di formazione professionali online durante il lockdown, possano usufruire di una sovvenzione pari al $95 \%$ delle spese sostenute per la formazione. ${ }^{78}$

\footnotetext{
${ }^{73} \mathrm{https}$ ://www.revenuquebec.ca/fr/maladie-a-coronavirus-covid-19/faq-pour-les-entreprises/

${ }^{74} \mathrm{https}: / /$ www.lepanierbleu.ca/

${ }^{75} \mathrm{https}: / /$ barc.ca.gov/whats-happening/news/santa-clara-county-explores-expansion-telecommuting-post-covid-19

${ }^{76} \mathrm{https}: / / a b c 7 n e w s . c o m / w o r k i n g-r e m o t e l y-f r o m-h o m e-t i p s-w o r k-j o b s-s a n t a-c l a r a-c o u n t y / 6151209 /$

https://www.mercurynews.com/2020/05/04/coronavirus-made-telework-necessary-santa-clara-county-to-exploreways-to-keep-it/

77https://www.netzpiloten.de/neue-provinz-forschungsprojekt-coworkland/

${ }^{78} \mathrm{http} / / / w w w . g 20$ e.tsinghua.edu.cn/EN/contents/1185/1304.html
} 
Stiria (Austria)

- Ampliamento del programma "Families! Friendly", con il quale la Camera del Lavoro della Stiria (AK) e l'Agenzia per la Promozione delle Imprese della Stiria (SFG) sostengono dal 2019 la creazione di posti di telelavoro (e di locali di lavoro più inclusivi) nelle piccole imprese. II programma si rivolge alle micro o piccole imprese con sede in Stiria. Gli investimenti ammissibili sono compresi tra i 2000 e i 50000 euro (fino a un massimo di 5000 euro per telelavoratore) e coprono le spese legate per la telefonia e internet, le attrezzature d'ufficio e commerciali, i telefoni cellulari e le infrastrutture presso la sede dell'azienda (ad esempio i server). La quota dei costi coperti è dell' $80 \%$ ( $50 \%$ da SFG più $30 \%$ da AK Styria). II programma promuove anche gli investimenti per spogliatoi separati sul posto di lavoro (obbligatori per le aziende con più di cinque dipendenti), al fine di sostenere l'assunzione di personale indipendentemente dal genere. ${ }^{79}$

- Durante la pandemia di COVID-19, SFG e AK Styria hanno lanciato un nuovo programma, chiamato "Telearbeit! Offensive", che amplia il campo d'azione di "Families! Friendly" in modo che possano fare domanda anche imprese di medie dimensioni. L'investimento minimo ammissibile è sceso a 1000 euro e il noleggio o il leasing di attrezzature è ora ammissibile. ${ }^{80}$

\section{Tokyo (Giappone)}

- Per frenare il contagio, il governo metropolitano di Tokyo ha offerto fino a 2,5 milioni di yen (circa 20500 euro) di aiuti finanziari alle PMI, e ad altre aziende, per il passaggio al telelavoro. La sovvenzione coprirà le spese sostenute per software e le attrezzature necessarie per il lavoro da remoto. Le imprese ammissibili devono avere meno di 1000 dipendenti e nessun pagamento arretrato delle imposte. ${ }^{81}$

\section{Tirolo (Austria)}

- Per contribuire a mantenere la produttività e garantire l'occupazione, il Tirolo sostiene le PMI nella creazione di posti di lavoro home-based. La sovvenzione consiste in un finanziamento una tantum non rimborsabile. I costi ammissibili al contributo sono i servizi di consulenza per l'ideazione e la configurazione delle attrezzature di hardware e software necessarie per la postazione di lavoro da casa (fino al 50\%), l'acquisto di software (fino al 50\%) e del relativo hardware (fino al 30\%, ma non più di 300 euro per ogni computer portatile, tablet, ecc.) I costi ammissibili devono ammontare ad almeno 500 euro e non possono superare i 5000 euro. ${ }^{82}$

\section{Tulsa, Oklahoma (Stati Uniti)}

- Tulsa Remote è un'iniziativa volta alla selezione e assunzione di lavoratori a distanza e nomadi digitali attraverso sovvenzioni del valore di 10000 dollari, un anno di iscrizione a uno spazio di coworking, supporto nell'identificazione di un alloggio e regolari opportunità di partecipare a iniziative di comunità. I candidati ammissibili devono risiedere al di fuori dell'Oklahoma. I selezionati

\footnotetext{
${ }^{79} \mathrm{https://www.sfg.at/f/lebenswerte-bueros/}$

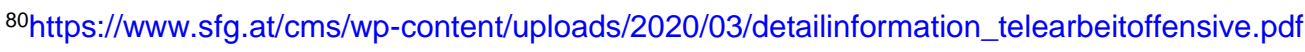

${ }^{81}$ https://metropolisjapan.com/covid-19-support-for-businesses/

${ }^{82} \mathrm{https}$ :/www.tirol.gv.at/arbeit-wirtschaft/wirtschaftsfoerderung/wirtschaftsfoerderungsprogramm/homeofficearbeitsplaetze/
} 
riceveranno una parte della sovvenzione in anticipo per sostenere le spese di trasloco, una parte sotto forma di uno stipendio mensile e il resto al termine del primo anno. ${ }^{83}$

\section{Vermont (Stati Uniti)}

- Dal 2019 il Vermont offre incentivi finanziari per attrarre telelavoratori nell'ambito del New Worker Relocation Grant Programme. ${ }^{84}$ I candidati ammissibili sono dipendenti a tempo pieno di aziende domiciliate al di fuori del Vermont. I candidati selezionati si trasferiranno e svolgeranno la maggior parte delle loro mansioni a distanza da un'abitazione o spazio di co-working situato nello Stato. I costi ammissibili per il rimborso includono il trasloco, strumenti software e hardware, l'accesso alla banda larga o il suo miglioramento, e l'abbonamento agli spazi di co-working o simili. Le sovvenzioni saranno concesse per il rimborso delle spese eleggibili. L'importo massimo della sovvenzione varia da 5000 a 7500 dollari, a seconda del luogo di destinazione del beneficiario. La piattaforma online dedicata, gestita dal Dipartimento per lo Sviluppo Economico del Vermont, include anche un ufficio di collocamento. ${ }^{85}$

\section{Vienna (Austria)}

- La Camera di Commercio Federale Austriaca ha creato una rete di sportelli per gli imprenditori autonomi di ciascuna delle nove province, cui offrono una vasta gamma di servizi. Uno dei più significativi consiste nella messa a disposizione di sale riunioni per gli imprenditori home-based. II WKO Forum Wien offre venti sale riunioni completamente attrezzate dal punto di vista tecnico, tra cui laptop, lavagne mobili e accesso a internet per i membri. È previsto anche un servizio gratuito di accudimento dei figli. L'utilizzo di queste sale riunioni è costantemente aumentato. Nel 2014 sono stati introdotti diversi webinar per rendere la formazione più accessibile ai membri con sede in zone rurali e periferiche. II loro contenuto comprende tematiche quali il marketing, vendite, social media e altre tematiche imprenditoriali di rilievo. Più di 8000 imprenditori in tutto il Paese hanno partecipato ai webinar. Questa modalità ha permesso loro di apprendere risparmiando tempo e costi di viaggio. ${ }^{86}$

\section{Wittenberge (Germania)}

- Nel 2019 la Città di Wittenberge, nel Brandeburgo, ha lanciato un'iniziativa pilota pubblico-privata volta ad attirare lavoratori creativi e del settore digitale. Mentre gli investitori privati forniscono le strutture dove lavorare (hanno ristrutturato un vecchio frantoio, trasformandolo in uno spazio di co-working), il Comune mette a disposizione le abitazioni (ha riqualificato le case sfitte e le offre in affitto a tariffe ridotte, oltre a pagare le spese legate all'ufficio). ${ }^{87} \mathrm{Nel}$ secondo semestre del 2020, 20 lavoratori del settore digitale su 60 candidati hanno avuto la possibilità di trasferirsi nello spazio di co-working di Wittenberge. ${ }^{88}$

\footnotetext{
${ }^{83} \mathrm{https}: / /$ tulsaremote.com/

${ }^{84} \mathrm{https}: / /$ accd.vermont.gov/press-releases/remote-worker-grant-program-set-launch

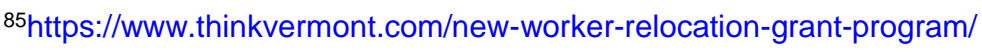

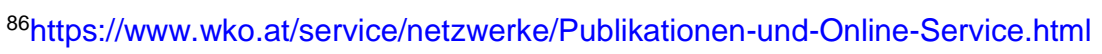

${ }^{87} \mathrm{https}$ :/www.spiegel.de/karriere/co-working-auf-dem-land-drinnen-arbeit-draussen-provinz-a-1281159.html

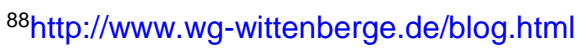




\section{Bibliografia}

American Enterprise Institute (2020), The Great American Land Rush of 2020, https://www.aei.org/research-products/report/the-great-american-land-rush-of-2020/ (accessed on 7 October 2020).

Barrero, J. et al. (2020), COVID-19 Is Also a Reallocation Shock, https://oui.doleta.gov/unemploy/claims arch.asp. (accessed on 6 October 2020).

Bloom, N. et al. (2014), "Does Working from Home Work? Evidence from a Chinese Experiment *", The Quarterly Journal of Economics, Vol. 130/1, pp. 165-218, http://dx.doi.org/10.1093/qje/qju032.

BMAS (2019), Anpacken Ergebnisbericht Handlungsempfehlungen, https://www.bmas.de/SharedDocs/Downloads/DE/PDF-Schwerpunkte/ergebnisberichtanpacken.pdf? blob=publicationFile\&v=2 (accessed on 26 May 2020).

Bonini, E. (2020), Telelavoro (ma anche intrattenimento): Confinamento più difficile al Sud, dove c'è minore accesso alla banda larga - Eunews, EUnews, https://www.eunews.it/2020/04/22/telelavoro-confinamento-piu-difficile-al-sud-ce-minoreaccesso-alla-banda-larga/129287 (accessed on 8 June 2020).

Brookings (2020), Telecommuting will likely continue long after the pandemic, https://www.brookings.edu/blog/up-front/2020/04/06/telecommuting-will-likely-continue-longafter-the-pandemic/ (accessed on 26 May 2020).

Brown, H. (2020), 10 ways e-residents are adapting their businesses during the crisis, https://medium.com/e-residency-blog/10-ways-e-residents-are-adapting-their-businessesduring-the-crisis-1f598c25070f (accessed on 1 June 2020).

Business Facilities (2020), U.S. Employees Working More Hours During COVID-19 Pandemic, https://businessfacilities.com/2020/03/u-s-employees-working-more-hours-during-covid-19pandemic/ (accessed on 26 May 2020).

Business Wire (2019), Tulsa Remote Launches Second Year, More than Doubles Participants to Receive \$10,000 / Business Wire, https://www.businesswire.com/news/home/20191029005377/en/Tulsa-Remote-LaunchesYear-Doubles-Participants-Receive (accessed on 3 June 2020).

Business Wire (2018), New Program in Tulsa, Oklahoma Offers Remote Workers $\$ 10,000$, Free [66] Co-working Space, Affordable Rent to Relocate to Tulsa / Business Wire, https://www.businesswire.com/news/home/20181113005771/en/New-Program-TulsaOklahoma-Offers-Remote-Workers (accessed on 1 June 2020). 
CFR (2020), A Double Pandemic: Domestic Violence in the Age of COVID-19 / Council on Foreign Relations, https://www.cfr.org/in-brief/double-pandemic-domestic-violence-age-covid19 (accessed on 29 June 2020).

Cgil/Fondazione di Vittorio (2020), Quando lavorare da casa è... SMART? $1^{\circ}$ Indagine Cgil/Fondazione Di Vittorio sullo Smart working, http://www.cgil.it/admin nv47t8g34/wpcontent/uploads/2020/05/Indagine Cgil-Fdv Smart working.pdf (accessed on 1 June 2020).

Confindustria (2020), Gli effetti sui lavoratori dello smart working, https://www.confindustria.it/home/centro-studi/temi-di-ricerca/valutazione-delle-politichepubbliche/tutti/dettaglio/Infografica-gli-effetti-sui-lavoratori-dello-smart-working (accessed on 8 June 2020).

Criteo (2020), Social Distancing Economy 2020: What \& How Consumers Buy in a New Normal I Criteo, https://www.criteo.com/insights/coronavirus-retail-trends/ (accessed on 8 June 2020).

Cushman \& Wakefield (2020), Looking Beyond COVID-19: Home/Remote working in China and its potential impact on office workplace strategy / Cushman \& Wakefield, https://www.cushmanwakefield.com/en/insights/covid-19/lessons-from-china/looking-beyondcovid-19-home-or-remote-working-in-china (accessed on 26 May 2020).

D'Alessandro J. (2020), Lavorare al sud a due passi dal mare, lo stipendio da Roma. Cosi l'Italia potrebbe cambiare - la Repubblica, la Repubblica, https://www.repubblica.it/dossier/tecnologia/rivoluzione-smartworking/2020/05/25/news/stipendio milanese ma lavorando al sud in riva al mare perch e il lavoro agile potrebbe cambiare radicalmente I italia-257433135/ (accessed on 8 June 2020).

DARES (2019), Quels sont les salariés concernés par le télétravail ?, https://dares.travailemploi.gouv.fr/IMG/pdf/dares analyses salaries teletravail.pdf (accessed on 3 June 2020).

Defilippis, E. et al. (2020), Collaborating during Coronavirus: the impact of COVID-19 on the nature of work, National Bureau of Economic Research, http://www.nber.org/papers/w27612 (accessed on 6 October 2020).

Der Spiegel (2019), Home Office: Ist ein Rechtsanspruch auf Heimarbeit Unsinn? - Der Spiegel, https://www.spiegel.de/karriere/home-office-ist-ein-rechtsanspruch-auf-heimarbeit-unsinn-a1289268.html (accessed on 26 May 2020).

Der Spiegel (2020b), Homeoffice-Boom: Corona-Krise stellt Zukunft des Büroturms infrage - Der Spiegel, https://www.spiegel.de/wirtschaft/soziales/buerotuerm-in-der-corona-krise-durchhomeoffice-boom-gefaehrdet-a-a38d96b0-6462-4fea-8900-5144e8f87c1e (accessed on 26 May 2020).

Der Spiegel (2020a), Recht auf Homeoffice: Zwangsbeglückung durch den Gesetzgeber - Der Spiegel, https://www.spiegel.de/karriere/recht-auf-homeoffice-zwangsbeglueckung-durchden-gesetzgeber-a-3c7c5ea5-0d42-4173-9655-87ca437e64df (accessed on 26 May 2020).

Dijkstra L. et al. (2018), The Geography of EU Discontent, Publications Office of the European Union, https://ec.europa.eu/regional policy/sources/docgener/work/2018 02 geog discontent.pdf (accessed on 1 June 2020). 
Dutcher G. (2012), The Effects of Telecommuting on Productivity: An Experimental Examination.

The Role of Dull and Creative Tasks,

https://www.researchgate.net/publication/257004501 The Effects of Telecommuting on Pr oductivity An Experimental Examination The Role of Dull and Creative Tasks (accessed on 22 June 2020).

Espinoza, R. and L. Reznikova (2020), "Who can log in? The importance of skills for the feasibility of teleworking arrangements across OECD countries", OECD Social, Employment and Migration Working Papers, No. 242, OECD Publishing, Paris, https://dx.doi.org/10.1787/3f115a10-en.

Eurofound (2020), Living, working and COVID-19: First findings - April 2020 / Eurofound, https://www.eurofound.europa.eu/publications/report/2020/living-working-and-covid-19-firstfindings-april-2020 (accessed on 30 July 2020).

Eurofound (2015), Sixth European Working Conditions Survey: 2015 / Eurofound, https://www.eurofound.europa.eu/surveys/european-working-conditions-surveys/sixtheuropean-working-conditions-survey-2015 (accessed on 26 May 2020).

European Centre for Disease Prevention and Control (2020), Coronavirus disease 2019 (COVID-19) pandemic: increased transmission in the EU/EEA and the UK - seventh update, https://www.ecdc.europa.eu/sites/default/files/documents/RRA-seventh-update-Outbreak-ofcoronavirus-disease-COVID-19.pdf (accessed on 3 June 2020).

Felstead, A. and D. Reuschke (2020), Homeworking in the UK: before and during the 2020 lockdown, Wales Institute of Social and Economic Research, https://wiserd.ac.uk/sites/default/files/documents/Homeworking\%20in\%20the\%20UK Report Final 3.pdf (accessed on 6 October 2020).

Gartner (2020), Gartner CFO Survey Reveals 74\% Intend to Shift Some Employees to Remote Work Permanently, https://www.gartner.com/en/newsroom/press-releases/2020-04-03gartner-cfo-surey-reveals-74-percent-of-organizations-to-shift-some-employees-to-remotework-permanently2 (accessed on 26 May 2020).

Global Workplace Analytics (2020), Work-at-Home After Covid-19_Our Forecast - Global Workplace Analytics, https://globalworkplaceanalytics.com/work-at-home-after-covid-19-ourforecast (accessed on 26 May 2020).

Golden, T., J. Veiga and R. Dino (2008), "The impact of professional isolation on teleworker job performance and turnover intentions: Does time spent teleworking, interacting face-to-face, or having access to communication-enhancing technology matter?", Journal of Applied Psychology, Vol. 93/6, pp. 1412-1421, http://dx.doi.org/10.1037/a0012722.

Guardian, T. (ed.) (2020), Women's research plummets during lockdown - but articles from men increase / Education / The Guardian, The Guardian, https://www.theguardian.com/education/2020/may/12/womens-research-plummets-duringlockdown-but-articles-from-men-increase (accessed on 9 July 2020).

Harding, R. (2020), Cities are too resilient to be killed by Covid, https://www.ft.com/content/ee05b075-1739-4eb7-b18b-70bd51fd1c47? shareType=nongift (accessed on 7 October 2020). 
ILO (2020b), ILO Policy Brief on COVID-19: Pillar 3: Protecting workers in the workplace, https://www.ilo.org/global/topics/coronavirus/impacts-and-responses/WCMS 739049/lang-en/index.htm (accessed on 3 June 2020).

ILO (2020a), ILO-UNESCO Joint Survey on Technical and Vocational Education and Training (TVET) and Skills Development during the time of COVID-19, https://www.ilo.org/wcmsp5/groups/public/--ed emp/documents/genericdocument/wcms 741397.pdf (accessed on 26 May 2020).

JRC (2020), Telework in the EU before and after the COVID-19: where we were, where we head to, https://ec.europa.eu/jrc/sites/jrcsh/files/jrc120945 policy brief covid and telework final.pdf (accessed on 4 November 2020).

Kaljulaid, K. (2018), Estonian President Kersti Kaljulaid reveals the future direction of eResidency / by Kaspar Korjus / E-Residency Blog, E-residentsuse blogi / Medium, https://medium.com/e-residency-blog/estonian-president-kersti-kaljulaid-reveals-the-futuredirection-of-e-residency-5b1177dfa78c (accessed on 30 July 2020).

Kurrle, L. et al. (2019), Remote Worker Grant Program Annual Report-Remote Worker Grant Program Annual Report Agency of Commerce and Community Development, https://legislature.vermont.gov/assets/Legislative-Reports/2019-Remote-Worker-ReportACCD.pdf (accessed on 22 June 2020).

LaRegione (2020), Al lavoro da casa devono essere riconosciute anche le spese - laRegione, https://www.laregione.ch/svizzera/svizzera/1439536/al-lavoro-da-casa-devono-esserericonosciute-anche-le-spese (accessed on 26 May 2020).

Livini, E. (2020), La Francia nazionalizza 10mila negozi per salvare i centri cittadini dall'ecommerce / Rep, Repubblica, https://rep.repubblica.it/ws/detail/generale/2020/07/03/news/la francia nazionanalizza 10mil a negozi per salvare i centri cittadini dall e-commerce-260875911/?ref=RHPPTP-BHI260894385-C12-P5-S4.4-T1 (accessed on 10 July 2020).

Löffler, R. et al. (2016), "Amenity Migration in the Alps: Applying Models of Motivations and Effects to 2 Case Studies in Italy", Mountain Research and Development, Vol. 36/4, pp. 484493, http://dx.doi.org/10.1659/mrd-journal-d-16-00042.1.

Mann, S. and L. Holdsworth (2003), "The psychological impact of teleworking: stress, emotions and health", New Technology, Work and Employment, Vol. 18/3, pp. 196-211, http://dx.doi.org/10.1111/1468-005x.00121.

McKinsey (2020a), COVID-19 and reskilling the workforce, https://www.mckinsey.com/businessfunctions/organization/our-insights/to-emerge-stronger-from-the-covid-19-crisis-companiesshould-start-reskilling-their-workforces-now (accessed on 1 June 2020).

McKinsey (2020b), How German "Mittelstand" copes with COVID-19 challenges, https://www.mckinsey.de/ /media/mckinsey/locations/europe\%20and\%20middle\%20east/deu tschland/news/presse/2020/2020-05-07\%20-\%20mittelstandsumfrage/covid19\%20mittelstandsumfrage\%20mckinsey\%202020.ashx (accessed on 1 June 2020).

McKinsey (2020c), Safeguarding Europe's livelihoods, https://www.mckinsey.com/industries/public-sector/our-insights/safeguarding-europeslivelihoods-mitigating-the-employment-impact-of-covid-19 (accessed on 1 June 2020). 
Messenger, J. et al. (2017), "Working anytime, anywhere: The effects on the world of work", http://dx.doi.org/10.2806/425484.

Muro M. (2020), Could Big Tech's move to permanent remote work save the American heartland?, Brookings, https://www.brookings.edu/blog/the-avenue/2020/05/26/could-bigtechs-move-to-permanent-remote-work-save-the-american-heartland/ (accessed on 8 June 2020).

Nafishah, O., Y. Shafiz Affendi Mohd and O. Wan Rozaini Sheik (2009), "A conflict between professional vs. domestic life? Understanding the use of ICT in teleworking for balance in work and family units" University of Wollongong in Dubai - Papers, pp. 3-15, https://ro.uow.edu.au/dubaipapershttps://ro.uow.edu.au/dubaipapers/126 (accessed on 7 October 2020).

Nationwide (2020), House price growth slows sharply as the impact of the pandemic begins to filter through, https://www.nationwide.co.uk/-/media/MainSite/documents/about/house-priceindex/2020/May 2020.pdf (accessed on 7 October 2020).

O'Connor Sarah (2020), Goodbye to the 'Pret economy' and good luck to whatever replaces it, Financial Times, https://www.ft.com/content/d8eb62ef-a1cb-4597-867b-15a79dbdcd5d (accessed on 7 October 2020).

ODOXA (2020b), Le Covid-19 bouleversera durablement le rapport au travail des Français Odoxa : Odoxa, http://www.odoxa.fr/sondage/covid-19-bouleverse-deja-modifieradurablement-rapport-francais-travail/ (accessed on 3 June 2020).

ODOXA (2020a), Télétravail : les Français tirent un premier bilan très positif malgré des problèmes d'équipements et des craintes sur la vie personnelle - Odoxa : Odoxa, http://www.odoxa.fr/sondage/teletravail-francais-tirent-premier-bilan-tres-positif-malgreproblemes-dequipements-craintes-vie-personnelle/ (accessed on 3 June 2020).

OECD (2019), Measuring the Digital Transformation: A Roadmap for the Future, OECD Publishing, Paris, https://dx.doi.org/10.1787/9789264311992-en.

OECD (2016), Be Flexible! Background brief on how workplace flexibility can help European employees to balance work and family, Paris Publishing, http://www.oecd.org/els/family/BeFlexible-Backgrounder-Workplace-Flexibility.pdf (accessed on 26 May 2020).

OECD (2015), The Metropolitan Century: Understanding Urbanisation and its Consequences, OECD Publishing, Paris, https://dx.doi.org/10.1787/9789264228733-en.

OECD (2020e), Capacity for remote working can affect lockdown costs differently across places - OECD, Paris Publication, https://read.oecd-ilibrary.org/view/?ref=134 134296u9iq2m67ag\&title=Capacity-for-remote-working-can-affect-lockdown-costs-differently-acrossplaces] (accessed on 19 June 2020).

OECD (2020d), From pandemic to recovery: Local employment and economic development -

$O E C D$, Paris Publishing, https://read.oecd-ilibrary.org/view/?ref=130 130810m60ml0s4wf\&title=From-pandemic-to-recovery-Local-employment-and-economicdevelopment (accessed on 26 May 2020).

OECD (2020c), OECD Employment Outlook 2020: Worker Security and the COVID-19 Crisis, OECD Publishing, Paris, https://dx.doi.org/10.1787/1686c758-en. 
OECD (2020b), Productivity gains from teleworking in the post COVID-19 era: How can public policies make it happen?, http://www.oecd.org/coronavirus/policy-responses/productivitygains-from-teleworking-in-the-post-covid-19-era-a5d52e99/ (accessed on 30 July 2020).

OECD (2020a), Supporting people and companies to deal with the COVID-19 virus - OECD, Paris Publishing, https://read.oecd-ilibrary.org/view/?ref=119 119686962r78x4do\&title=Supporting people and companies to deal with the Covid -19 virus (accessed on 26 May 2020).

Office for National Statistics (2020), Technology intensity and homeworking in the UK - Office for National Statistics, https://www.ons.gov.uk/employmentandlabourmarket/peopleinwork/employmentandemployee types/articles/technologyintensityandhomeworkingintheuk/2020-05-01 (accessed on 3 June 2020).

Parkhomenko A. et al. (2020), How do cities change when we work from home?, https://www.andrii-parkhomenko.net/files/DKP Covid Urban.pdf (accessed on 1 June 2020).

Reuschke, D. and M. Domecka (2018), "Policy Brief on Home-Based Businesses", OECD SME and Entrepreneurship Papers, No. 11, OECD Publishing, Paris, https://dx.doi.org/10.1787/abfe755f-en.

Startup Poland (2016), The Polish Tech Scene. 5 years, https://www.home.startuppoland.org/ (accessed on 22 June 2020).

The Baltic Times (2019), E-residency program has brought Estonia EUR $31 \mathrm{~m} / \mathrm{n}$ in direct income, https://www.baltictimes.com/eresidency program has brought estonia eur $31 \mathrm{mln}$ in direct income/ (accessed on 3 June 2020).

The Japan Times (2020), What will happen to inclusion in COVID-19's wake? | The Japan Times, https://www.japantimes.co.jp/opinion/2020/05/11/commentary/japan-commentary/willhappen-inclusion-covid-19s-wake/\#.XtTL7GgzZPY (accessed on 1 June 2020).

The Times (2020), Empty Italian hills to come alive after exodus from cities / News / The Times, https://www.thetimes.co.uk/article/empty-italian-hills-to-come-alive-after-exodus-from-citiesnrxhtnrod (accessed on 26 May 2020).

Upwork (2020), Economist Report: Remote Workers on the Move, https://www.upwork.com/press/releases/economist-report-remote-workers-on-the-move.

US Chamber of Commerce (2020), Small Business Coronavirus Impact Poll / U.S. Chamber of Commerce, https://www.uschamber.com/report/small-business-coronavirus-impact-poll (accessed on 3 June 2020).

US Office of Personnel Management (2017), Status of Telework in the Federal Government Report to Congress.

V. Reeves R. Rothwell J. (2020), Class and COVID: How the less affluent face double risks, Brookings, https://www.brookings.edu/blog/up-front/2020/03/27/class-and-covid-how-the-lessaffluent-face-double-risks/ (accessed on 8 June 2020). 
Valentino-DeVries J. et al. (2020), Location Data Says It All: Staying at Home During Coronavirus Is a Luxury - The New York Times, The New York Times, https://www.nytimes.com/interactive/2020/04/03/us/coronavirus-stay-home-rich-poor.html (accessed on 8 June 2020).

VOX EU (2020), COVID-19, teleworking, and productivity / VOX, CEPR Policy Portal, https://voxeu.org/article/covid-19-teleworking-and-productivity (accessed on 3 June 2020). 